\title{
An optical view of the filament region of Abell $85^{\star, \star \star}$
}

\author{
G. Boué ${ }^{1}$, F. Durret ${ }^{1}$, C. Adami ${ }^{2}$, G. A. Mamon ${ }^{1}$, O. Ilbert ${ }^{3,2}$, and V. Cayatte ${ }^{4}$ \\ 1 Institut d'Astrophysique de Paris (UMR 7095: CNRS \& Université Pierre et Marie Curie), 98bis Bd Arago, 75014 Paris, France \\ e-mail: boue@iap.fr \\ 2 LAM, Pôle de l'Étoile, Site de Château-Gombert, 38 rue Frédéric Joliot-Curie, 13388 Marseille Cedex 13, France \\ 3 Institute for Astronomy, 2680 Woodlawn Dr., University of Hawaii, Honolulu, Hawaii 96822, USA \\ 4 Observatoire de Paris, section Meudon, LUTH, CNRS-UMR 8102, Université Paris 7, 5 Pl. Janssen, 92195 Meudon, France
}

Received 31 October 2007 / Accepted 23 June 2008

ABSTRACT

\begin{abstract}
Aims. We present an optical investigation of the Abell 85 cluster filament $(z=0.055)$ previously interpreted in X-rays as groups falling on to the main cluster. We compare the distribution of galaxies with the X-ray filament, and investigate the galaxy luminosity functions in several bands and in several regions. We search for galaxies where star formation may have been triggered by interactions with intracluster gas or tidal pressure due to the cluster potential when entering the cluster.

Methods. Our analysis is based on images covering the South tip of Abell 85 and its infalling filament, obtained with CFHT MegaPrime/MegaCam $\left(1 \times 1 \mathrm{deg}^{2}\right.$ field $)$ in four bands $\left(u^{*}, g^{\prime}, r^{\prime}, i^{\prime}\right)$ and ESO $2.2 \mathrm{~m} \mathrm{WFI}\left(38 \times 36 \mathrm{arcmin}^{2}\right.$ field $)$ in a narrow band filter corresponding to the redshifted $\mathrm{H} \alpha$ line and in an $R_{\mathrm{C}}$ broad band filter. The LFs are estimated by statistically subtracting a reference field. Background contamination is minimized by cutting out galaxies redder than the observed red sequence in the $g^{\prime}-i^{\prime}$ versus $i^{\prime}$ colour-magnitude diagram.

Results. The galaxy distribution shows a significantly flattened cluster, whose principal axis is slightly offset from the X-ray filament. The analysis of the broad band galaxy luminosity functions shows that the filament region is well populated. The filament is also independently detected as a gravitationally bound structure by the Serna \& Gerbal (1996, A\&A, 309, 65) hierarchical method. 101 galaxies are detected in the $\mathrm{H} \alpha$ filter, among which 23 have spectroscopic redshifts in the cluster, 2 have spectroscopic redshifts higher than the cluster and 58 have photometric redshifts that tend to indicate that they are background objects. One galaxy that is not detected in the $\mathrm{H} \alpha$ filter probably because of the filter low wavelength cut but shows $\mathrm{H} \alpha$ emission in its SDSS spectrum in the cluster redshift range has been added to our sample. The 24 galaxies with spectroscopic redshifts in the cluster are mostly concentrated in the South part of the cluster and along the filament.

Conclusions. We find a number of galaxies showing evidence for star formation in the filament, and all our results are consistent with the previous hypothesis that the X-ray filament in Abell 85 is a gravitationally bound structure made of groups falling on to the main cluster.
\end{abstract}

Key words. galaxies: clusters: individual: Abell 85 - galaxies: luminosity functions, mass function

\section{Introduction}

Cosmological simulations of the large-scale structure of the Universe display the filamentary nature of the large-scale galaxy distribution (e.g. Springel et al. 2005), and the observed largescale galaxy distribution is consistent with this picture, even though filaments are more difficult to see in redshift space (e.g. Pimbblet 2005). X-ray observations of the nearby rich cluster Abell 85 highlight a filament of hot gas extending towards the

\footnotetext{
* Based on observations obtained with: 1) MegaPrime/MegaCam, a joint project of CFHT and CEA/DAPNIA, at the Canada-France-Hawaii Telescope (CFHT) which is operated by the National Research Council (NRC) of Canada, the Institut National des Sciences de l'Univers of the Centre National de la Recherche Scientifique (CNRS) of France, and the University of Hawaii; 2) the European Southern Observatory; 3 ) the Anglo-Australian Observatory. This work is also based in part on data products produced at TERAPIX and the Canadian Astronomy Data Centre as part of the Canada-France-Hawaii Telescope Legacy Survey, a collaborative project of NRC and CNRS. We have also made use of the NASA/IPAC Extragalactic (NED) Database.

$\star \star$ Appendix A is only available in electronic form at

http://www . aanda.org
}

South East to near one virial radius (Durret et al. 1998b, 2003, 2005).

One would obviously like to know if this X-ray filament can be traced in the galaxy distribution. If so, one would expect that this filament would be a preferential route for the infall of galaxies onto the cluster, even within the virialised region of the cluster. The influence of infall is not always well understood, except for a few clusters, such as e.g. Coma (Adami et al. 2007). One would also like to know if the filament region follows the same morphology-density relation as seen in clusters (Dressler 1980; Dressler et al. 1997), or whether the filament constitutes a special environment. Similarly, do the galaxies in the filament dipslay the same specific rates of star formation as seen in other cluster regions of the same density, or is the star formation enhanced or quenched? Indeed, star formation can be triggered when the groups of the filament enter the cluster or dense areas, due to environmental effects such as ram pressure from the intracluster gas or tidal pressure due to the cluster potential (Bekki 1999).

The galaxy population in the X-ray filament is easily traced in maps of the projected distribution of galaxies up to a given apparent magnitude limit and with a selection in redshifts to remove obvious cluster outliers. Alternatively, galaxy luminosity functions in several wavebands are a good tool to sample the 
Table 1. Observation characteristics: coordinates, exposure times in seconds (seeing in arcseconds), observation dates, and program Id.

\begin{tabular}{|c|c|c|c|c|c|c|}
\hline $\begin{array}{l}\text { Image center coordinates } \\
00^{\mathrm{h}} 41^{\mathrm{m}} 56.5^{\mathrm{s}}-09^{\circ} 53^{\prime} 56^{\prime \prime}\end{array}$ & $\begin{array}{c}u^{*} \\
13440\left(1.32^{\prime \prime}\right)\end{array}$ & $\begin{array}{c}g^{\prime} \\
7480\left(1.42^{\prime \prime}\right)\end{array}$ & $\begin{array}{c}r^{\prime} \\
4550\left(1.45^{\prime \prime}\right)\end{array}$ & $\begin{array}{c}i^{\prime} \\
4200\left(0.87^{\prime \prime}\right)\end{array}$ & $\begin{array}{c}\text { Dates } \\
10 / 2004\end{array}$ & $\begin{array}{c}\text { Program Id } \\
04 \mathrm{BF} 02\end{array}$ \\
\hline $\begin{array}{l}\text { Image center coordinates } \\
00^{\mathrm{h}} 41^{\mathrm{m}} 39.8^{\mathrm{s}}-09^{\circ} 35^{\prime} 15^{\prime \prime}\end{array}$ & $\begin{array}{c}R_{\mathrm{C}} \\
2900\left(0.93^{\prime \prime}\right)\end{array}$ & $\begin{array}{c}\mathrm{H} \alpha \\
5800\left(0.85^{\prime \prime}\right)\end{array}$ & & & $\begin{array}{c}\text { Dates } \\
11 / 2004\end{array}$ & $\begin{array}{c}\text { Program Id } \\
\text { 074.A-0029B }\end{array}$ \\
\hline
\end{tabular}

history of the faint galaxy population (e.g. Adami et al. 2007, and references therein) including star formation history, evolutionary processes and environmental effects. In particular, the faint-end slopes of galaxy luminosity functions (LFs) in clusters of galaxies have been observed in some cases to vary with clustercentric distance and are expected to be influenced by physical processes (mergers, tides) affecting cluster galaxies (as summarized e.g. by Boué et al. 2008, hereafter B08).

The $\mathrm{H} \alpha$ line is a good indicator of star formation and has been detected in a number of galaxies in nearby clusters. The first pioneering work on this topic was due to Moss et al. (1988), Moss \& Whittle (1993), and Moss et al. (1998), who performed the first $\mathrm{H} \alpha$ surveys in a sample of clusters with an objective prism. Based on this survey, Moss et al. (1998) and Moss \& Whittle (2000) analyzed tidally induced star formation in several clusters; they found spatial variations, both within a cluster and from one cluster to another: starburst emission in spirals increases from regions of lower to higher density, and from clusters with lower to higher central galaxy space density. Moss $\&$ Whittle (2005) were then able to show that the frequency of emission line galaxies (ELGs) is similar for field and cluster galaxies of all types, and that for galaxies of a given morphological type the fraction of ELGs is independent of environment. A large $\mathrm{H} \alpha$ survey was performed on several nearby clusters by Boselli et al. (2002) and Gavazzi et al. (2002, 2006). They analyzed several trends with radius and found in particular that luminous galaxies show a decrease in their average $\mathrm{H} \alpha$ equivalent width in the inner $\sim 1$ virial radius, while low-luminosity galaxies do not show this trend. Large $\mathrm{H} \alpha$ surveys have also allowed to estimate $\mathrm{H} \alpha$ luminosity functions and star formation rates in some of these clusters (Iglesias-Páramo et al. 2002; Umeda et al. 2004). Observations of clusters in $\mathrm{H} \alpha$ have also revealed some interesting features. For example, a few $\mathrm{H} \alpha$ tails and filaments as well as intracluster HII regions have been detected in a few clusters such as Abell 1795 (Crawford et al. 2005), Coma (Yagi et al. 2007) or Abell 3627 (Sun et al. 2007). A starbursting compact group was also found to be falling on to Abell 1367, where complex trails of ionized gas behind the galaxies were detected (Cortese et al. 2006).

We present here a detailed optical analysis of the filament region of Abell 85. This cluster is at a redshift of 0.055 and shows a very complex structure in X-rays, with a main cluster, a South blob and an extended filament (discovered in X-rays) at least $4 \mathrm{Mpc}$ in length. Based on ROSAT PSPC and XMM-Newton data, evidence was found for several merging episodes, one of these still ongoing, as suggested by the interpretation of the X-ray filament as groups falling on to the main cluster (Durret et al. 1998b, 2003, 2005). However, the optical properties of the galaxies composing the X-ray filament have not been analyzed until now; they may give us clues on the physical properties of this filament and on the likelihood of the merging scenario described above.

We have obtained two sets of data: ESO $2.2 \mathrm{~m}$ WFI $38 \times 36 \operatorname{arcmin}^{2}$ images in a narrow band filter corresponding to the wavelength of $\mathrm{H} \alpha$ at the cluster redshift and in a broad band $R_{\mathrm{C}}$ filter to subtract the continuum contribution, covering the South half of Abell 85 and its filament, and deep $1 \times 1 \mathrm{deg}^{2}$ field images obtained at CFHT with MegaPrime/MegaCam in four bands $\left(u^{*} g^{\prime} r^{\prime} i^{\prime}\right)$ covering the South tip of Abell 85 and the infalling filament. Both sets of data sample the filament feeding the cluster from the Southeast, and the impact region where the filament is believed to be hitting the cluster itself (this impact region is indeed hotter in X-rays). The virial radius, defined as the radius where the mean mass density is 100 times the critical density of the Universe, is 0.65 , derived by extrapolating the radius of overdensity 500 given by Durret et al. (2005). Thus, our Megacam images (not centered on the cluster) cover part of Abell 85 and more distant regions, well beyond the virial radius. The Sloan Digital Sky Survey (SDSS) covers the region of Abell 85. We have retrieved all the redshifts available in the SDSS to build a large redshift catalogue for the region of Abell 85, as well as all the galaxy spectra in the region covered by our WFI data.

The paper is organized as follows. We present our Megacam and WFI data and data reduction in Sect. 2. In Sect. 3, we describe our results on $\mathrm{H} \alpha$ imaging and discuss the spatial distribution and properties of $\mathrm{H} \alpha$ emitting galaxies, together with properties derived from the SDSS data. In Sect. 4, we present our results obtained for the LF in the four broad photometric bands. In Sect. 5, we discuss our results concerning the LFs in terms of large scale environmental effects on the cluster galaxy populations. Final conclusions are drawn in Sect. 6.

We assume a distance of $242.2 \mathrm{Mpc}$ to Abell 85 $\left(H_{0}=71 \mathrm{~km} \mathrm{~s}^{-1} \mathrm{Mpc}^{-1}, \Omega_{\mathrm{m}}=0.27\right.$ and $\left.\Omega_{\Lambda}=0.73\right)$. The distance modulus is 36.92 and the scale is $1.055 \mathrm{kpc} \operatorname{arcsec}^{-1}$. We give magnitudes in the $\mathrm{AB}$ system. At this distance, the Megacam field of view corresponds to $3.8 \times 3.8 \mathrm{Mpc}^{2}$, while the virial radius is $2.5 \mathrm{Mpc}$.

\section{Observations and data reduction}

\subsection{Megacam data reduction}

Abell 85 was observed at CFHT with the large field MegaPrime/MegaCam camera in October 2004, program 04BF02, P.I. F. Durret (see Table 1). The deep $1 \times 1 \mathrm{deg}^{2}$ field images obtained at CFHT with MegaPrime/MegaCam in four bands $\left(u^{*} g^{\prime} r^{\prime} i^{\prime}\right)$ cover the South tip of Abell 85 and the infalling filament. These images were reduced by the Terapix pipeline using the standard reduction tool configuration. We refer the reader to http: //terapix.iap. fr/ for reduction details.

Object extraction was made using the SExtractor package (Bertin \& Arnouts 1996) in double-image mode. The CFHTLS pipeline at the Terapix data center creates a $\chi^{2}$ image based upon the quadratic sum of the images in the different wavebands. Objects are then detected on this image. In contrast with the CFHTLS images, our set of $u^{*} g^{\prime} r^{\prime} i^{\prime}$ images for Abell 85 presents important differences in their PSFs (see Table 1). For this reason, we chose a different approach from that of the Terapix data center: instead of considering the $\chi^{2}$ image as the reference image, we use the band with the best seeing in our data: $i^{\prime}$. Detections were performed in this band and object characteristics were 


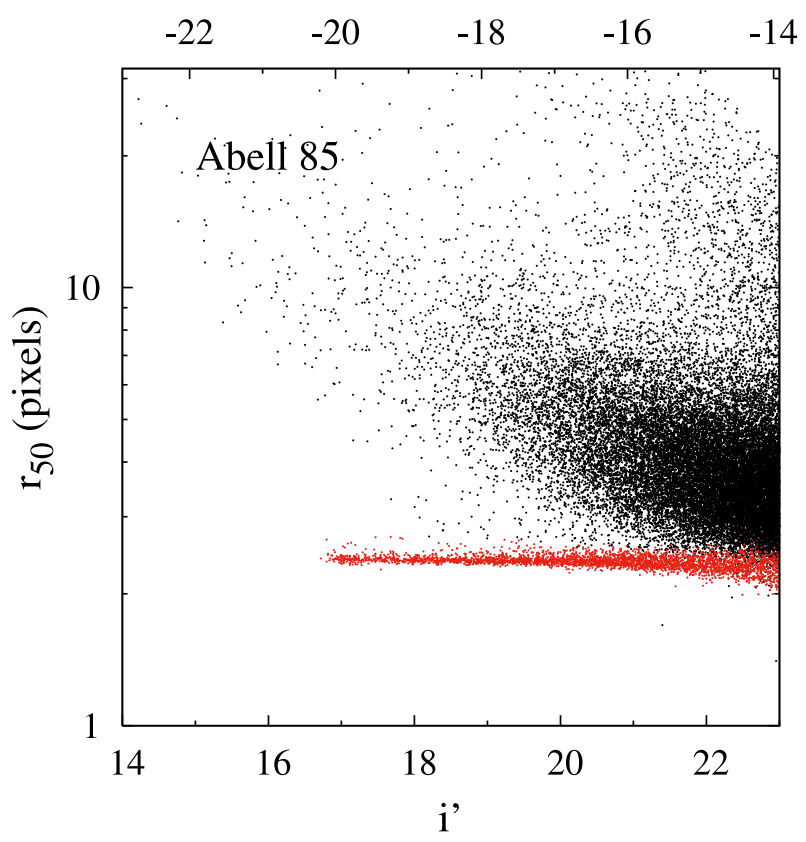

Fig. 1. Star-galaxy separation for the Megacam data: half-light radius versus apparent magnitude (bottom axis) and absolute magnitude (top axis) of the detections on the Abell 85 image. Objects assumed to be stars are plotted in red.

measured in all bands. The detections and measures were made using the CFHTLS parameters, among which an absolute detection threshold of 0.4 ADUs, a minimal detection area of 3 pixels and a $7 \times 7$ pixel Gaussian convolution filter of 3 pixels of $F W H M$. In the output catalog, we only kept objects with semi-minor axes larger than 1 pixel and mean surface brightness within the half-light radius greater than $\mu=26.25$ in order to remove artefacts.

A detailed description of the way the completeness levels and reliabilities of our detections were estimated using simulations, as well as the star-galaxy separation (see Figs. 1 and 2), magnitude corrections for Galactic extinction and estimate of the useful area covered by the Megacam images $\left(0.785 \mathrm{deg}^{2}\right)$ can be found in B08.

For the computation of the luminosity functions, we used the CFHTLS Deep (D1, D2, D3 and D4, i.e. 4 MegaCam fields) and Wide (W1, W2 and W3, 59 MegaCam fields) as comparison field data, as described in B08. Note that we re-extracted object catalogues from each of the $4 \times 4$ Deep Field (DF) images by making the detections in the $i^{\prime}$ band, as for Abell 85 .

Regions of the CFHTLS observed more than once (common areas of 19 Megacam fields) were considered to estimate the magnitude uncertainties as a function of magnitude in an external way (see B08, Fig. 4).

\subsection{WFI data reduction}

Imaging observations were performed in service mode with the ESO $2.2 \mathrm{~m}$ telescope and the WFI camera (program 074.A0029B, P.I. F. Durret) during the nights of $31 / 10 / 2004$ to $02 / 11 / 2004$. The images cover an area of $38 \times 36 \mathrm{arcmin}^{2}$ with a pixel scale of $0.238 \mathrm{arcsec} / \mathrm{px}$, covering the South half of Abell 85 and its filament. They were taken in a narrow band filter corresponding to the wavelength of $\mathrm{H} \alpha$ at the cluster redshift (ESO \#869) and in a broad band $R_{\mathrm{C}}$ filter (ESO \#844) to
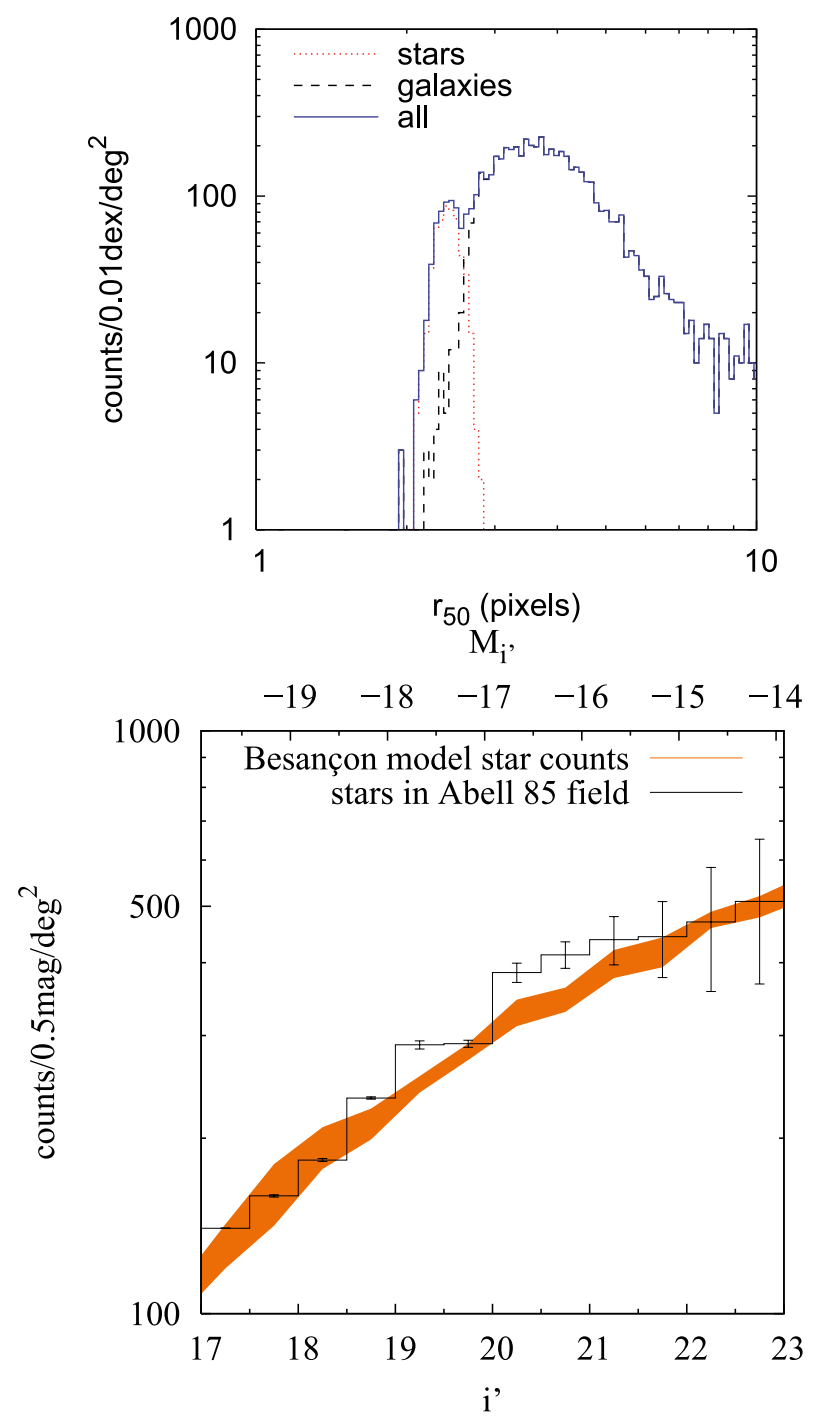

Fig. 2. Megacam data. Top: histogram of all detections for Abell 85 in the range $22<i^{\prime}<22.5$ with the distributions of stars and galaxies obtained using our star-galaxy separation. Bottom: comparison of star counts obtained with the Besançon model (Robin et al. 2003) (orange shaded region) and from the Abell 85 image (black histogram) using our star-galaxy separation.

subtract the continuum contribution. The response curves of these two filters are shown in Fig. 3.

The images were corrected for bias and flat field in the usual way. They were then combined and astrometrically corrected by Bertin, in order to obtain a final image in each filter. These images were calibrated photometrically based on observations in the $R_{\mathrm{C}}$ filter of the SA 113, LATPHE and RU 149 standard star fields from the Landolt (1992) list observed during the same nights.

Object detections were made on the $R_{\mathrm{C}}$ image with the SExtractor software. The star-galaxy separation was performed as for the Megacam data. We tested our photometric calibration by cross-identifying 680 stars with magnitudes $17<R_{\mathrm{C}}<21$ and comparing their $R_{\mathrm{C}}$ magnitudes with their $r^{\prime}$ magnitudes measured in the Megacam image. We find an average value $\left\langle r^{\prime}-R_{\mathrm{C}}\right\rangle=0.210$. Fukugita et al. (1995) give $r^{\prime}-R_{\mathrm{C}}=0.25$, 0.22 and 0.17 for elliptical, Scd and Im galaxies respectively. Therefore our WFI $R_{\mathrm{C}}$ band and Megacam $r^{\prime}$ band photometric calibrations are in good agreement. 


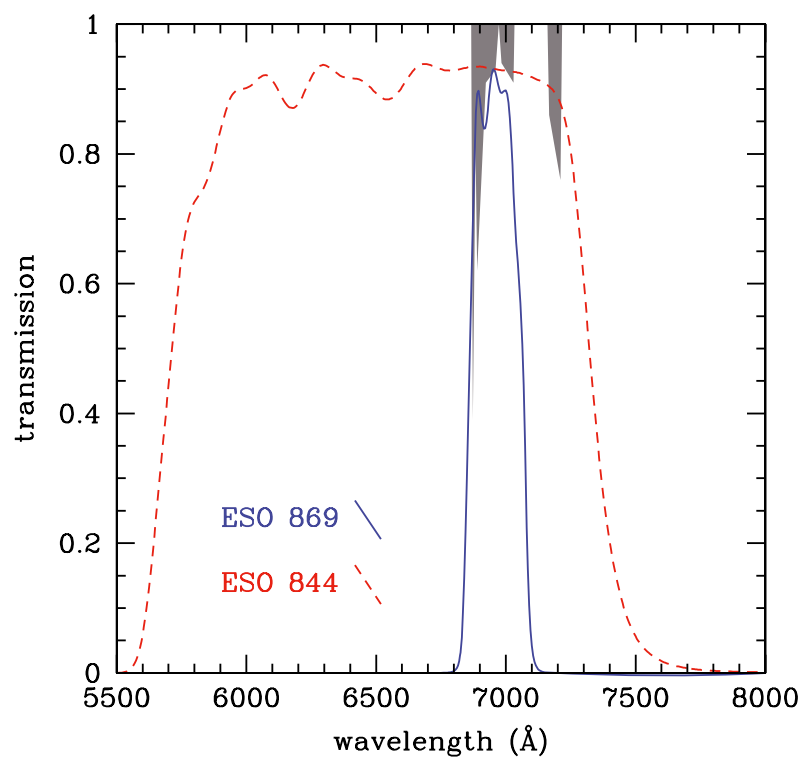

Fig. 3. Filter transmission for the narrow band (solid line) and broad band (dotted line) filters used with the ESO $2.2 \mathrm{~m}$ and WFI camera. Also shown in grey is the position of telluric absorption inferred from a twilight spectrum.

We then ran SExtractor in double image mode on the $\mathrm{H} \alpha$ image, based on the detections made in the $R_{\mathrm{C}}$ band. The magnitudes of the same 680 stars were then measured, giving the average magnitude difference $\left\langle\mathrm{H} \alpha-R_{\mathrm{C}}\right\rangle=2.37$ mag between the $\mathrm{H} \alpha$ and the $R_{\mathrm{C}}$ filters, with a dispersion of \pm 0.13 mag. This relation allowed us to calibrate the $\mathrm{H} \alpha$ image.

Note that the [NII] $\lambda 6548,6584$ lines are also included in the $\mathrm{H} \alpha$ filter. However, they should not contribute more than $\sim 20 \%$ to the total emission line flux (e.g. Cortese et al. 2004).

The $\mathrm{H} \alpha$ observations can encounter two potential problems. First, a strong telluric line is present near $6900 \AA$, that is towards the left wing of the ESO \#869 filter (see Fig. 3), and its absorption is non negligible up to about $6911 \AA$, which corresponds to the wavelength of $\mathrm{H} \alpha$ redshifted by 0.053 . The redshift interval for cluster membership was estimated to be about [0.0451-0.0657] by Durret et al. (1998b). Therefore the telluric line may lead to underestimate the contribution of emission line galaxies in the [0.045-0.053] redshift range. In the region covered by our WFI data, there are 373 measured redshifts in our complete redshift catalogue of 1705 objects (see Sect. 2.4). Out of these, 220 have redshifts in the cluster range, and 173 are in the [0.053-0.0657] interval. Therefore, $220-173=47$ galaxies are potentially affected by the telluric line, representing $21 \%$ of the cluster galaxies. On the other hand, the effect of telluric absorption at 6900 and $7200 \AA$ on broad band imaging can be considered as negligible, since these telluric lines are expected to affect the $R_{\mathrm{C}}$ filter by less than $10 \%$, given the breadth of the $R_{\mathrm{C}}$ filter.

Second, the ESO \#869 filter is centered on wavelength $6963 \AA$ and has a width of $207 \AA^{1}$. Considering again that the redshift range for Abell 85 is [0.0451-0.0657], the wavelength of the redshifted $\mathrm{H} \alpha$ line for a galaxy at the lower redshift limit of 0.0451 is $6858.8 \AA$; at this wavelength, the filter transmission is about $40 \%$. For a redshift of 0.049 , the wavelength of the redshifted $\mathrm{H} \alpha$ line becomes $6884.38 \AA$ and at this wavelength

\footnotetext{
${ }^{1} \mathrm{http}$ ://wWw.1s.eso.org/lasilla/sciops/2p2/E2p2M/WFI/ filters
}

the filter transmission is about $85 \%$. Therefore, we can consider that the galaxies that will be affected by this filter cut are those with redshifts between 0.045 and 0.049 . The correction for incompleteness in our $\mathrm{H} \alpha$ detections due to this filter cut is quite uncertain because the exact position of the filter shoulders shifts in wavelength with ambient temperature. Besides, as discussed below, our redshift catalogue is based in part on SDSS data for which we have no accurate completeness estimate. We will therefore not attempt to correct for incompleteness.

\subsection{AAOmega spectroscopic data}

Spectroscopy was obtained with the Anglo-Australian Telescope in November 2006 (P.I. G.A. Mamon). Objects were selected inside the Megacam area between $g^{\prime}=18$ and 21.5 (computed inside the AAOmega 2 arcsec diameter fiber area). The exposure times were $4700 \mathrm{~s}$ for the brightest targets and $6500 \mathrm{~s}$ for the faintest targets. Details on the spectroscopic run will be given in a forthcoming paper. These spectra were only used here to increase our redshift catalogue.

\subsection{SDSS spectroscopic data and full redshift catalogue}

Since the region of Abell 85 was covered by the SDSS, many redshifts are available for this area in the NED database in addition to the Durret et al. (1998a) redshift catalogue. Additional spectroscopic redshifts obtained at the AAT were added to the redshift catalogue extracted from the NED database, in order to have a redshift catalogue as complete as possible to cross correlate with $\mathrm{H} \alpha$ detections. The complete catalogue contains 1705 objects, out of which 506 galaxies have redshifts in the [0.0451-0.0657] cluster interval; out of the latter 220 are in the WFI field.

We extracted all the spectra available in the Sixth Data Release of the SDSS (SDSS-DR6) within the area covered by our WFI image, and selected those with an equivalent width in the $\mathrm{H} \alpha$ line larger than $3 \AA$. We found 12 objects in this sample, among which 6 are in the cluster redshift range. Five of these galaxies are detected in our $\mathrm{H} \alpha$ image, and the only one which is not is ACO85J004127.86-092329.54 is at a redshift of 0.0494 , which is probably cut by the $\mathrm{H} \alpha$ filter, since its spectrum unambigously shows $\mathrm{H} \alpha$ emission (see its spectrum in the Appendix). Quantities derived from the $\mathrm{H} \alpha$ flux for this galaxy would only correspond to the SDSS spectroscopic aperture, while for the other objects these quantities are integrated throughout the galaxy, so we will not compute them. The spectra of the 6 SDSS galaxies with $\mathrm{H} \alpha$ emission in their spectra and within the cluster redshift range are shown in the Appendix.

\section{Large-scale galaxy distribution}

The large-scale distribution of SDSS-DR6 galaxies around Abell 85 is shown in Fig. 4. Around the cluster, the distribution of the SDSS galaxies having a spectroscopic redshift \pm 0.005 around that of Abell 85 and inside the virial radius has an axis ratio of $0.58 \pm 0.05$ along position angle $\mathrm{PA}=164^{\circ} \pm 4^{\circ}$, counted anticlockwise from north. These measurements were done using the 2 nd order moments of the particle distribution (e.g., Bertin 2006), and the errors were estimated using 1000 bootstraps. Therefore, the cluster is highly and significantly flattened. Even if the SDSS is not complete (in particular because of fiber crowding), the regions with few SDSS galaxies at the cluster redshift are filled with background SDSS galaxies, so the flattening of 


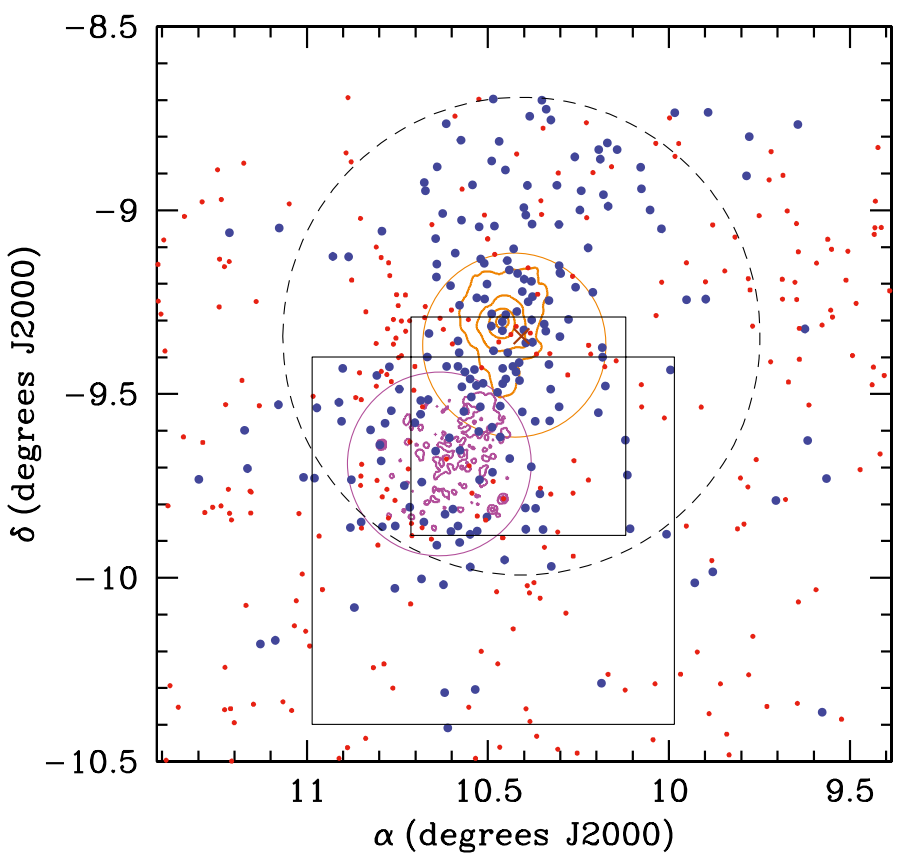

Fig. 4. Large-scale distribution of SDSS-DR6 galaxies around Abell 85. Large blue and small red filled circles represent cluster members (within $1500 \mathrm{~km} \mathrm{~s}^{-1}$ from the cluster mean velocity) and outliers, respectively. The huge dashed circle represents the virial radius. The small and large squares show the WFI and MegaCam fields, respectively. The region $-8^{\circ} 7<\delta<-8.5$ was not covered by the SDSS. XMM-Newton logspaced X-ray contours and fields are shown in orange (main cluster) and purple (filament, with contour levels 1.1, 4 and 11 times lower than the lowest one of the main cluster).

the cluster galaxy distribution does not appear to be caused by incompleteness. Moreover, Fig. 4 shows that the flattening of the cluster is aligned with the X-ray filament discovered by Durret et al. (1998b), in particular with the axis linking the cluster X-ray centre to the extended region of the filament at $\alpha=10.55$ and $\delta=-9.55$ (roughly at 0.4 virial radii from the cluster X-ray centre).

\section{Galaxy luminosity functions}

We now present and discuss the properties of the galaxy luminosity functions in the various Megacam bands and in different regions of the cluster. A full description of the method applied to derive luminosity functions (LFs) and the importance of applying a colour cut to eliminate background galaxies can be found in B08.

Figure 5 shows the galaxy distribution in a colour-magnitude diagram. The reddest galaxies of the cluster lie in the red sequence (its upper limit, as estimated in B08, is marked in red). All galaxies above this line are redder and are assumed to be field objects.

We computed LFs both for the whole Megacam field of view and for 16 subfields. The subfields define a regular square grid of $15 \times 15 \mathrm{arcmin}^{2}$ each and allow a good compromise between spatial resolution and uncertainties in individual magnitude bins. We used 1 mag bins to limit the uncertainties. Several subregions are then defined including a certain number of subfields with common properties.

The overall LF in the four photometric bands is displayed in Fig. 6 (we remind the reader that the cluster center is located outside the surveyed field). At bright magnitudes, the LFs have

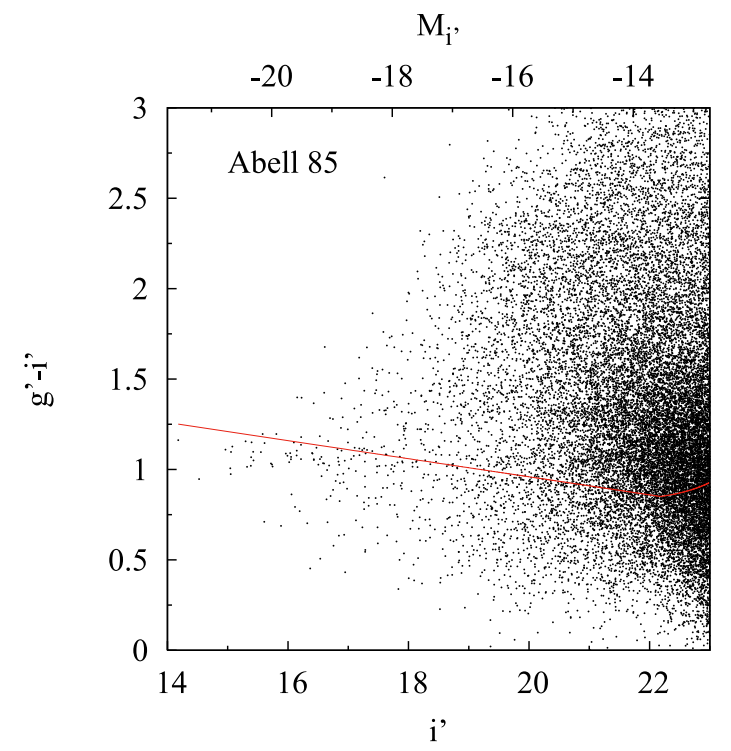

Fig. 5. $\left(g^{\prime}-i^{\prime}\right)$ versus $i^{\prime}$ colour-magnitude plot for the objects detected with Megacam. The red line shows the limit colour-magnitude relation applied to select possible cluster members when drawing the luminosity functions.
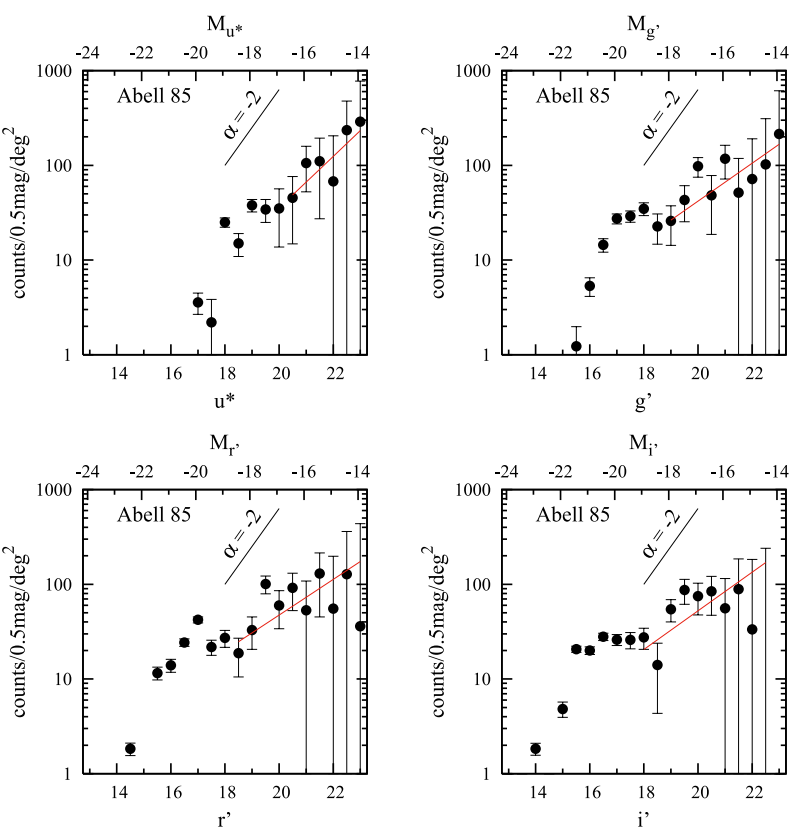

Fig. 6. Global luminosity functions for the total area covered by Megacam (i.e. the filament and "impact" region of Abell 85) in the four bands with the best power law fits shown in red.

comparable shapes in the $g^{\prime}, r^{\prime}$ and $i^{\prime}$ bands, while there are fewer galaxies in the $u^{*}$ band.

The LF in $15 \times 15 \operatorname{arcmin}^{2}$ subfields is displayed in Fig. 7, showing that the LFs of Abell 85 are not similar over the whole field. Some subfields are very poorly populated, while others exhibit rising LFs. As expected, the subfields towards the Southeast of the image (coinciding with the X-ray filament and its continuation) are more densely populated than the Southwest, implying that the cluster (or the filament) extends far beyond the virial radius.

We can therefore divide the cluster into three main regions: the North zone where the cluster still dominates, the Southeast rectangle and the Southwest rectangle (respectively in blue, red 
Table 2. Galaxy luminosity function in four bands: power-law fits on global image.

\begin{tabular}{lcccc}
\hline \hline Filter & $u^{*}$ & $g^{\prime}$ & $r^{\prime}$ & $i^{\prime}$ \\
\hline Magnitude range & $20.5 \leq u^{*} \leq 23.0$ & $19.0 \leq g^{\prime} \leq 23.0$ & $18.5 \leq r^{\prime} \leq 23.0$ & $18.0 \leq i^{\prime} \leq 22.5$ \\
$\alpha \pm 1 \sigma$ & $-1.70 \pm 0.48$ & $-1.46 \pm 0.15$ & $-1.52 \pm 0.12$ & $-1.45 \pm 0.11$ \\
\hline
\end{tabular}

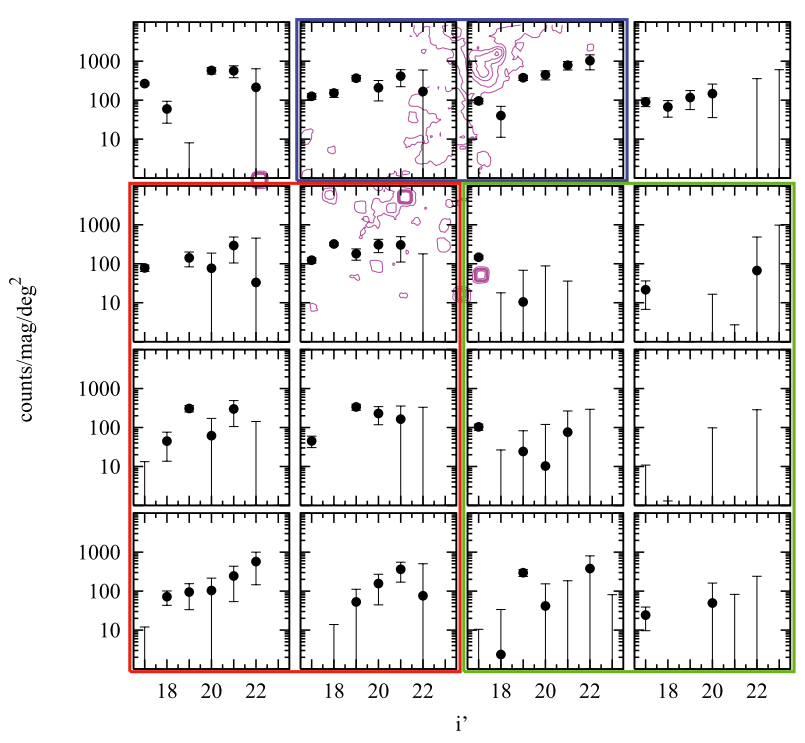

Fig. 7. Luminosity functions for the filament region of Abell 85 in the $i^{\prime}$ band. Each subfield is $15 \times 15 \mathrm{arcmin}^{2}$. X-ray intensity contours with logarithmic steps from XMM-Newton data are superimposed. Three main areas are defined: in blue (the South tip of the cluster and "impact" region), red (containing the filament) and green (where only a few objects are detected).

and green in Fig. 7). The LFs in these three subregions, in the four photometric bands, are displayed in Fig. 8. As expected, we can see significantly populated LFs in the zones that also show cluster X-ray emission (the North and South east regions, and the filament) while there are hardly any galaxies in the Southwest region. Note that the southern edge of the North region corresponds to a distance to the cluster center of about $1.9 \mathrm{Mpc}$, less than the virial radius $(2.5 \mathrm{Mpc})$.

Though there are significantly positive points in the two bestpopulated areas (the North and filament zones), the LFs do not display very well-defined power-law slopes. We notice, however, that the shapes of the LFs in the North and Southeast regions (respectively the blue and red regions in Fig. 8) are quite similar. The Southwest area is obviously much less populated, since there are very few significant points in its LFs.

\section{Dynamical properties of the filament}

We estimated galaxy types from their colours in the four Megacam bands, based on the Le Phare photometric redshift technique developed by Arnouts and Ilbert (Zucca et al. 2006). Galaxies are thus divided into four types: type 1 for ellipticals, type 2 for early type spirals, type 3 for intermediate spirals and type 4 for late type spirals. Note that this classification was applied only to the spectroscopic sample, by fitting the template with a fixed redshift, and is therefore quite robust. This classification corresponds to the Coleman et al. (1980) templates, adjusted to the colours as described by Zucca et al. (2006) (also see http://www.ifa.hawaii.edu/ ilbert/ these.pdf.gz, pages 50 and 142).
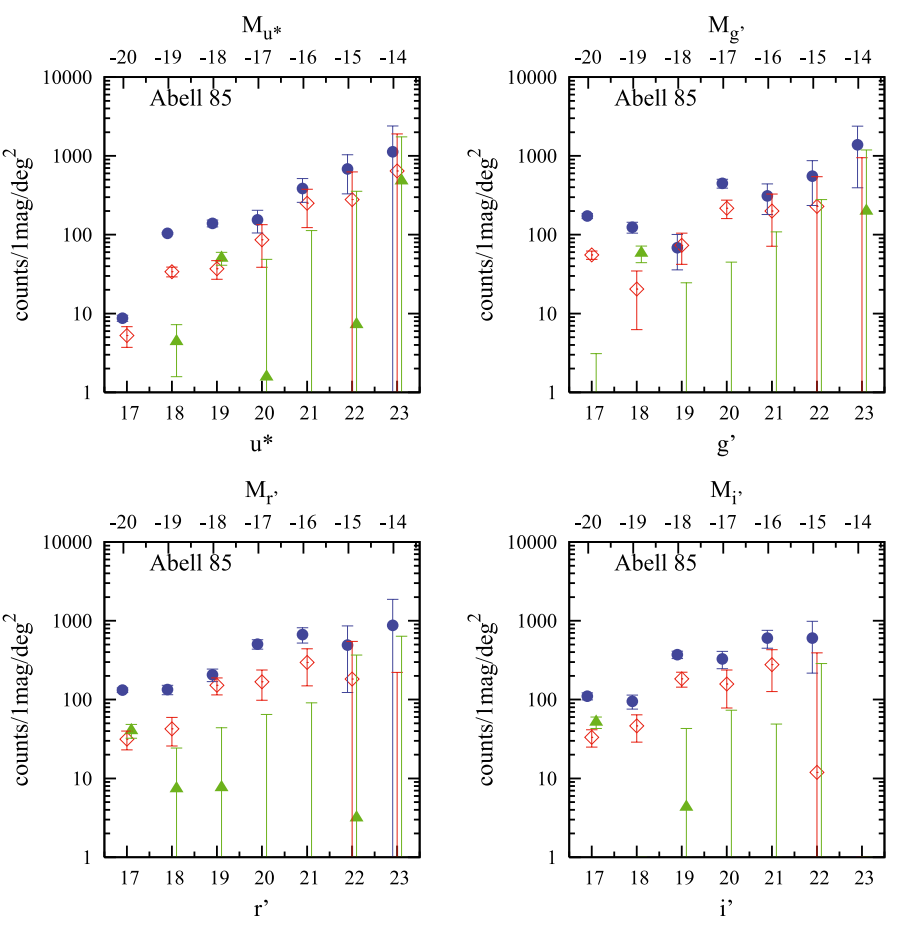

Fig. 8. Luminosity functions in the four bands for Abell 85 in the three main areas described in Fig. 7. The colours of the points are: blue for the North rectangle (South tip of the cluster and "impact" region), red for the Southeast rectangle, and green for the Southwest rectangle.

The distributions of galaxies in the cluster redshift range [0.0451-0.0657] with different symbols for the various types are shown in Fig. 9. Very few type 2 (early type) galaxies were found in our sample. Late type spirals appear to follow more or less the filament, or at least to be located in the East half of the image, whereas intermediate type spirals are distributed throughout.

Such a distribution could be explained if late type spirals arrive on to the cluster from the East, more or less along the filament and are then transformed into earlier type spirals which then gravitate inside the cluster (see e.g. Adami et al. 1999, and references therein). This scenario, although speculative, would agree with the fact that a VLA survey of Abell 85 has shown that the majority of the galaxies detected in HI were in the Eastern half of the cluster (Bravo-Alfaro et al. 2008). The galaxies located in the Western half of the cluster would then have lost their HI through ram pressure stripping. More detailed modelling is required however to fully understand this phenomenon.

Out of our global redshift catalogue of 1705 objects with spectroscopic redshifts, we created a catalogue of 181 galaxies with redshifts in the [0.0451-0.0657] range and located within our Megacam image, and applied to this catalogue the Serna \& Gerbal (1996) hierarchical method (hereafter SG). This method allows to extract galaxy subgroups from a catalogue containing positions, magnitudes and redshifts, based on the calculation of their (negative) binding energies. The output is a list of galaxies belonging to the selected group, as well as the 


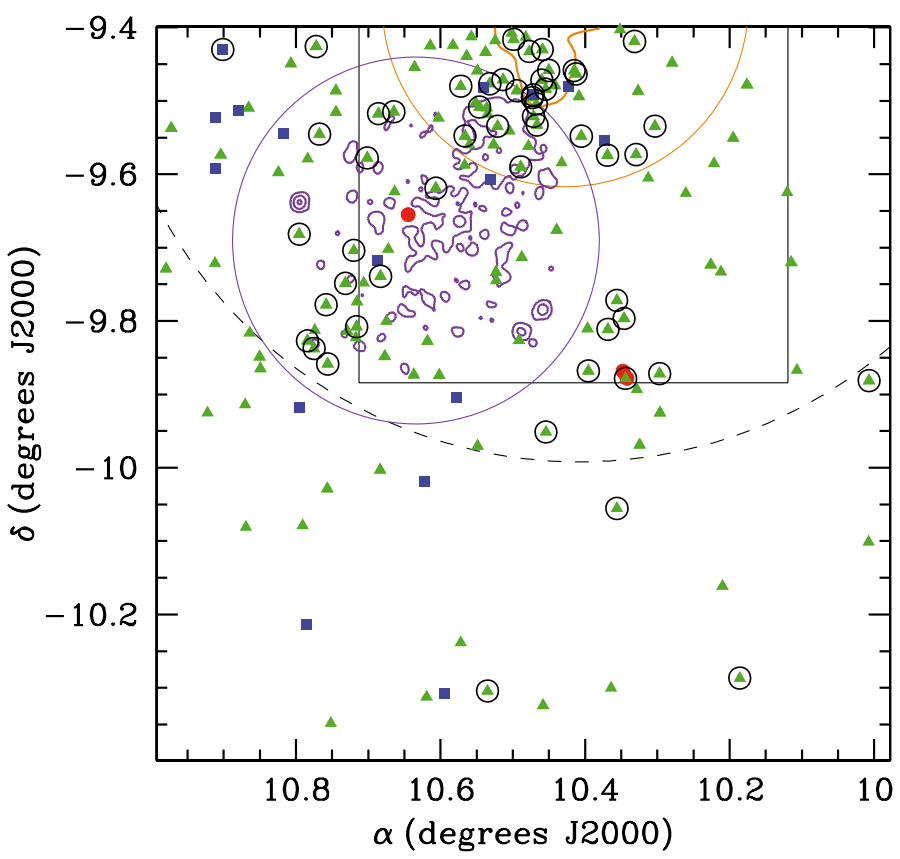

Fig. 9. Same as Fig. 4 for galaxies in the cluster redshift range [0.0451-0.0657], highlighting the different galaxy types and dynamical substructures they belong to. The symbols are the following: filled red circles for ellipticals, filled green triangles for intermediate type spirals, and blue squares for late type spirals. Large black circles show the galaxies belonging to the dynamically bound substructure.

Table 3. Completeness of redshift catalogue in the $\mathrm{H} \alpha$ image field.

\begin{tabular}{rrrc}
\hline \hline$R_{\mathrm{C}}$ & $N_{\text {with } z}$ & $N_{\text {total }}$ & completeness $(\%)$ \\
\hline $13-14$ & 1 & 1 & 100 \\
$14-15$ & 8 & 8 & 100 \\
$15-16$ & 22 & 22 & 100 \\
$16-17$ & 52 & 55 & 94.5 \\
$17-18$ & 68 & 74 & 91.9 \\
$18-19$ & 78 & 160 & 48.8 \\
$19-20$ & 34 & 366 & 9.3 \\
$20-21$ & 21 & 875 & 2.4 \\
$21-22$ & 12 & 1532 & 0.8 \\
$22-23$ & 2 & 1553 & 0.1 \\
\hline
\end{tabular}

information on the binding energy of the group itself. We assumed an $M / L_{\mathrm{R}}=200$; note however that, as shown e.g. by Adami et al. (2005) for Coma galaxies, results derived from the SG method are not sensitive to the exact choice of $M / L$ (taking $M / L_{\mathrm{R}}=400$ instead of 200 did not change our results).

The SG method confirms the existence of a dynamically bound structure roughly following the filament, the brightest galaxy in the filament being the elliptical seen in Fig. 9. The mass (i.e. the sum of the galaxy masses) of this dynamically bound system is about $6.6 \times 10^{12} M_{\odot}$, in the range of groups of galaxies. Therefore, the physical existence of the filament is consistent with the output of the SG analysis.

A second rather loose substructure is also found by the SG method towards the Southwest. This substructure also includes an elliptical galaxy, which is brighter by about $0.5 \mathrm{mag}$ than the other 8 galaxies in that zone, and has a mass of about $3.9 \times 10^{11} M_{\odot}$.

One of the limitations of the SG method is the completeness of the redshift catalogue. We give in Table 3 the number of galaxies with redshifts in the field of the $\mathrm{H} \alpha$ image and the total number of galaxies in that region in magnitude bins of 1 mag. This table shows that the redshift catalogue is more than $90 \%$ complete up to $R_{\mathrm{C}}=18$, and still almost $50 \%$ complete in the $R_{\mathrm{C}}=[18,19]$ mag bin; above $R_{\mathrm{C}}=19$ the completeness decreases rapidly. The substructures found with the SG method are therefore very likely to be true, since gravitational effects are dominated by massive (i.e. bright) galaxies, but the numbers of objects included in each substructure and the corresponding substructure masses should only be considered as indicative.

\section{Emission line galaxies}

\subsection{Sample definition}

In order to select $\mathrm{H} \alpha$ emitting galaxies, we first created a "pure" emission line image (implicitly assuming that, in average, the spectra of stars are flat in the wavelength region covered by the filters), based on the relation found in Sect. 2.2: $\left\langle\mathrm{H} \alpha-R_{\mathrm{C}}\right\rangle=2.37 \mathrm{mag}$. The "pure" $\mathrm{H} \alpha$ image was therefore obtained by subtracting $10^{-0.4 \times 2.37} \times I\left(R_{\mathrm{C}}\right)=0.113 I\left(R_{\mathrm{C}}\right)$ to the $\mathrm{H} \alpha$ image, where $I\left(R_{\mathrm{C}}\right)$ is the intensity of the $R_{\mathrm{C}}$ image.

However, when we tried to find $\mathrm{H} \alpha$ emitting objects by drawing a colour-magnitude diagram of the "net" $\mathrm{H} \alpha$ flux as a function of the $R_{\mathrm{C}}$ magnitude, and selecting galaxies with net $\mathrm{H} \alpha$ fluxes higher than 4 (or even more) times the dispersion, at least 20 or $30 \%$ of the selected objects were spurious (i.e. they were not visible on the "pure" emission line image).

We were thus led to modify our extraction of $\mathrm{H} \alpha$ detections in two ways:

1) since the sensitivity of the WFI camera is not perfectly uniform throughout the field, we modeled the spatial variations of the parameter $n$ (defined as the ratio of the $\mathrm{H} \alpha$ to $R_{\mathrm{C}}$ fluxes) around its mean value of 0.113 with Zernike polynomials of order 0 to 8 (which include terms up to the 4 th power in angular distance). This fit was based on all the stars present in the image;

2) since the seeings were not exactly the same in the $\mathrm{H} \alpha$ and $R_{\mathrm{C}}$ images (see Table 1 ), we smoothed the $R_{\mathrm{C}}$ subimages to match the seeing of the $\mathrm{H} \alpha$ subimages. Our smoothing filter was a Gaussian of $F W H M$ equal to the root difference of the squares of the two $F W H M$ s. We then subtracted the smoothed $R_{\mathrm{C}}$ subimages multiplied by their respective values of $n$ to the corresponding $\mathrm{H} \alpha$ subimages to create "pure" $\mathrm{H} \alpha$ subimages.

The Zernike fit reduced the rms residuals in $n$ from 0.0169 to 0.0163 (an $F$ test indicates that this small reduction in rms for the 7 extra fit parameters is highly significant, given the 492 stars that the fit was performed on), while the individual stars displayed variations of $n$ roughly 35 times larger than the typical measurement errors ( $n$ is significantly correlated to the $g-i$ colour of the star). Nevertheless, the Zernike fit shown in Fig. 10, indicates $10 \%$ relative large-scale variations of the $\mathrm{H} \alpha$ to $R_{\mathrm{C}}$ ratio.

The detection of $\mathrm{H} \alpha$ emitters was then made by running SExtractor on these "pure" $\mathrm{H} \alpha$ subimages, with a $2 \sigma$ limit detection (note that this corresponds to a detection level of $3.3 \sigma$ with the usual definition, since SExtractor applies a smoothing) and requesting at least 12 pixels connected to each other to detect an object. 105 galaxies were detected in this way. The "pure" $\mathrm{H} \alpha$ subimages corresponding to these 105 galaxies were visually inspected and 5 objects were discarded, three because they were too close to a bright star, one was close to a CCD edge and one was a large galaxy with only very weak diffuse $\mathrm{H} \alpha$ emission (if any). 


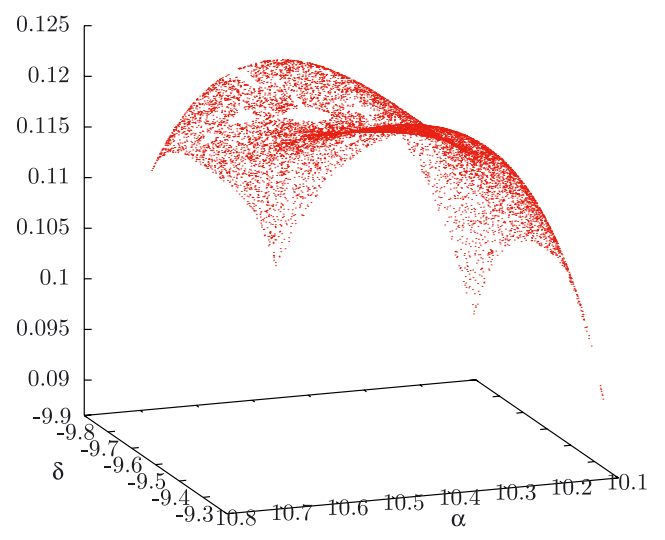

Fig. 10. Fit by Zernike polynomials of the $\mathrm{H} \alpha$ to $R_{\mathrm{C}}$ flux ratio for stars throughout the WFI field.

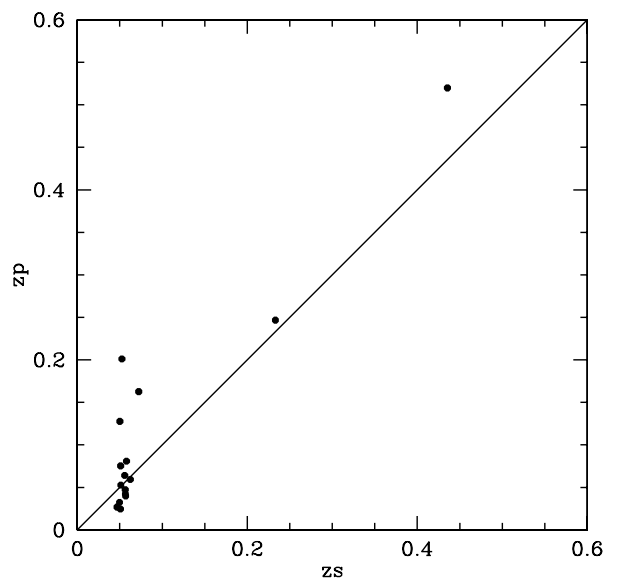

Fig. 11. Photometric redshifts estimated with the Le Phare software versus spectroscopic redshifts for the 17 galaxies detected in $\mathrm{H} \alpha$ and having both spectroscopic and photometric redshifts.

For the 101 remaining objects, considered as detected in the $\mathrm{H} \alpha$ filter, fluxes were then measured by running again SExtractor on the $\mathrm{H} \alpha$ and smoothed $R_{\mathrm{C}}$ subimages and measuring their respective fluxes in the area where $\mathrm{H} \alpha$ emission was detected in the "pure" $\mathrm{H} \alpha$ subimages. The calculations of $\mathrm{H} \alpha$ fluxes and equivalent widths and of the errors on these quantities are explained in Sect. 6.3.

\subsection{Cluster membership}

Cluster membership is obviously a crucial issue in this study. We constructed a redshift catalogue of 1705 objects in the area of Abell 85 (see Sect. 2.3), among which 373 are in the WFI field. Out of the 101 galaxies detected in the $\mathrm{H} \alpha$ filter, 25 have redshifts: 23 are in the [0.0451-0.0723] cluster redshift range (note that this range was chosen to include the galaxy with a redshift of 0.0723 , slightly above the cluster range), and the remaining two galaxies have redshifts 0.2331 and 0.4355 .

In order to test whether the 76 galaxies detected in $\mathrm{H} \alpha$ but with no spectroscopic redshift were likely to belong to the cluster, we estimated photometric redshifts (hereafter $z_{\text {phot }}$ ). This was only possible for the objects covered by our Megacam images in four broad bands (see Fig. 14). A plot of photometric versus spectroscopic redshifts for the 17 galaxies with both spectroscopic and photometric redshifts is shown in Fig. 11. This figure shows that galaxies belonging to the cluster from their spectroscopic redshifts are all at $z_{\text {phot }}<0.22$.
Table 4. Range of redshifts where lines enter our $\mathrm{H} \alpha$ filter.

\begin{tabular}{lcc}
\hline \hline Line & Wavelength $(\AA)$ & Redshifts \\
\hline Ly $\alpha$ & 1216 & $4.62-4.84$ \\
{$[\mathrm{OII}]$} & 3737 & $0.83-0.90$ \\
{$[\mathrm{OIII}]$} & 5007 & $0.36-0.42$ \\
$\mathrm{H} \beta$ & 4861 & $0.40-0.46$ \\
$\mathrm{H} \alpha$ & 6563 & $0.04-0.08$ \\
{$[\mathrm{NII}]$} & 6584 & $0.04-0.08$ \\
\hline
\end{tabular}

Among the $76 \mathrm{H} \alpha$ emitting galaxies with no spectroscopic redshift, 58 have photometric redshifts. The remaining 18 galaxies have no photometric redshift either because they are outside the Megacam field or because they are too close to a bright star, in a region masked in the Megacam image analysis. Out of the 58 galaxies with photometric redshifts, all have $z_{\text {phot }}>0.2$.

Among the 58 galaxies detected in $\mathrm{H} \alpha$ and with only photometric redshifts, it is a little surprising to find no galaxy with $z_{\text {phot }}<0.2$, though photometric redshifts should be considered as mainly giving a statistical information. Our recent experience when applying photometric redshifts to the Abell 85 and Abell 496 four band Megacam catalogues before obtaining spectroscopic redshifts (for galaxies with typical magnitudes $r=20-21$ ) has shown that roughly $50 \%$ of the galaxies selected with $z_{\text {phot }}<0.2$ were actually at $z_{\text {spec }}<0.2$. Note however that this fraction of $50 \%$ would have been reduced if we had used the galaxies observed in the SDSS to "train" the photometric redshift software. On the other hand, less than $15 \%$ of the galaxies with $z_{\text {phot }}>0.2$ were in fact found to be cluster members in this study.

Can our $\mathrm{H} \alpha$ detections be contaminated by background objects? The filter transmission of the ESO \#869 filter is 5\% at $\lambda_{1} \sim 6830 \AA$ and $\lambda_{2} \sim 7100 \AA$. Table 4 shows the range of redshifts for which different astronomical lines can be detected in our $\mathrm{H} \alpha$ filter. Besides the $\mathrm{H} \alpha$-[NII] lines at $z \sim 0.06 \pm 0.02$, the emission detected in our narrow band filter can also be due to $\mathrm{H} \beta$ $(z=0.43 \pm 0.03)$ or [OIII] $\lambda 4959,5007$ at $0.39 \pm 0.03$, [OII] $\lambda 3727$ at $0.83<z<0.90$ or $\operatorname{Ly} \alpha$ at $4.62<z<4.84$.

Murayama et al. (2007) have estimated the mean density of Ly $\alpha$ emitters at redshift $z \sim 5$.7. For the mean redshift at which we would detect Ly $\alpha$ galaxies $(z=4.73)$, with our cosmological parameters, the distance modulus is 48.3. The faintest galaxy in our sample for which we detect line emission in the ESO \#869 filter has a magnitude $R_{\mathrm{C}}=21$. Therefore at $z=4.73$ this would correspond to a galaxy of absolute magnitude $M_{R_{\mathrm{C}}}=-27.3$, i.e. $280 L *$ according to the Schechter fit to the SDSS luminosity function by Blanton et al. (2003), an unlikely value. We therefore believe that the contamination of our sample by Ly $\alpha$ galaxies is negligible.

Takahashi et al. (2007) have detected 3176 [OII] $\lambda 3727$ emitting galaxies at $z \sim 1.2$ in a volume of $5.5 \times 10^{5} \mathrm{Mpc}^{3}$. The corresponding density is $5.77 \times 10^{-3} \mathrm{Mpc}^{-3}$. In the ESO \#869 filter, [OII] emitting galaxies will be detected if their redshifts are between 0.8324 and 0.9049 . With our cosmology, these redshifts correspond to luminosity distances of 5279 and $5857 \mathrm{Mpc}$ respectively. The mean angular distance at the mean redshift of 0.8687 is $1594 \mathrm{Mpc}$. Therefore the volume filled by [OII] galaxies in the field of our WFI image is $2.0 \times 10^{5} \mathrm{Mpc}^{3}$, leading to a total number of galaxies of 1149 . However, as mentioned above, we detect $\mathrm{H} \alpha$ emission only for galaxies brighter than $R_{\mathrm{C}}=21$, corresponding roughly to $i \sim 20.6$. We retrieved 

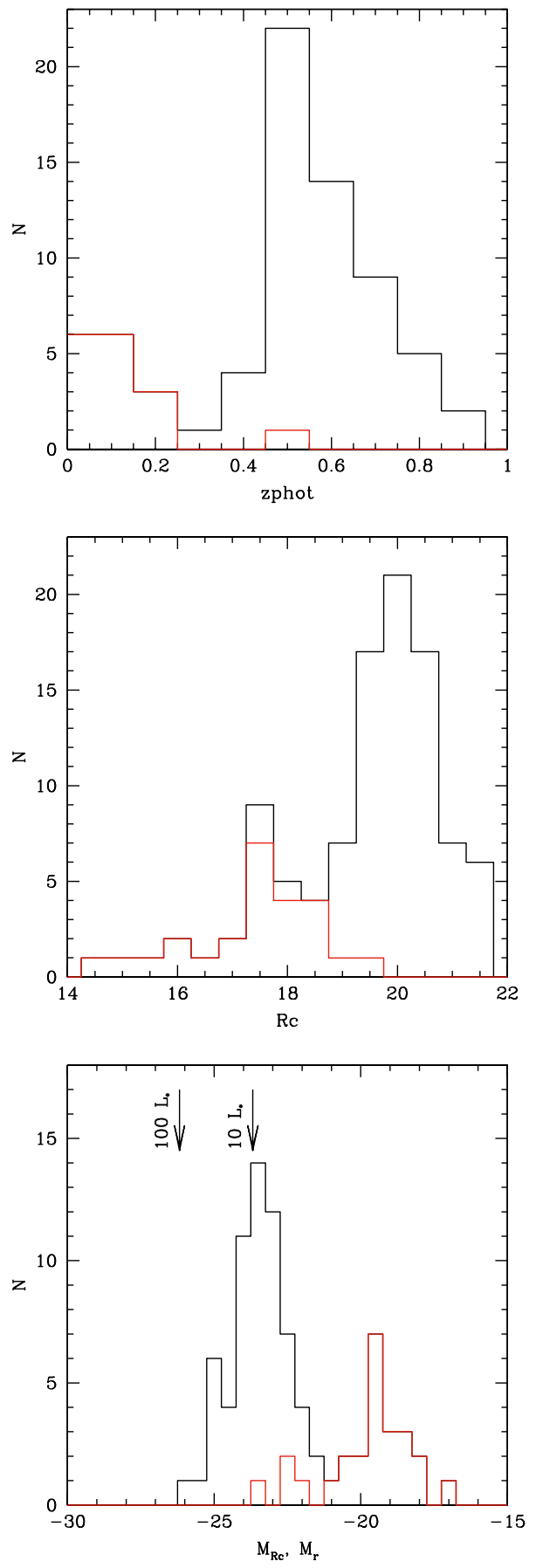

Fig. 12. Top: histograms of the photometric redshifts for the 50 galaxies detected in $\mathrm{H} \alpha$ without spectroscopic redshifts (black) and for the 17 galaxies which also have spectroscopic redhifts (red). Middle: histogram of the apparent magnitudes of these objects. Bottom: histogram of the corresponding absolute magnitudes if the galaxies are at the distances given by their photometric redshifts. The vertical arrows indicate the absolute magnitudes corresponding to $10 L_{*}$ and $100 L_{*}$.

the Takahashi et al. (2007) catalogue and found that no galaxy is brighter than $i=20.6$ (the brightest one is at $i=21.15$; this is a magnitude measured in a 3 arcsec aperture, but since these are distant objects their total magnitudes are probably not very different). Therefore the number of [OII] contaminants in our $\mathrm{H} \alpha$ filter is expected to be negligible too.

As for $\mathrm{H} \beta$ or [OIII] emitters at respective redshifts of about 0.38 and 0.42 , the histogram of the photometric redshifts

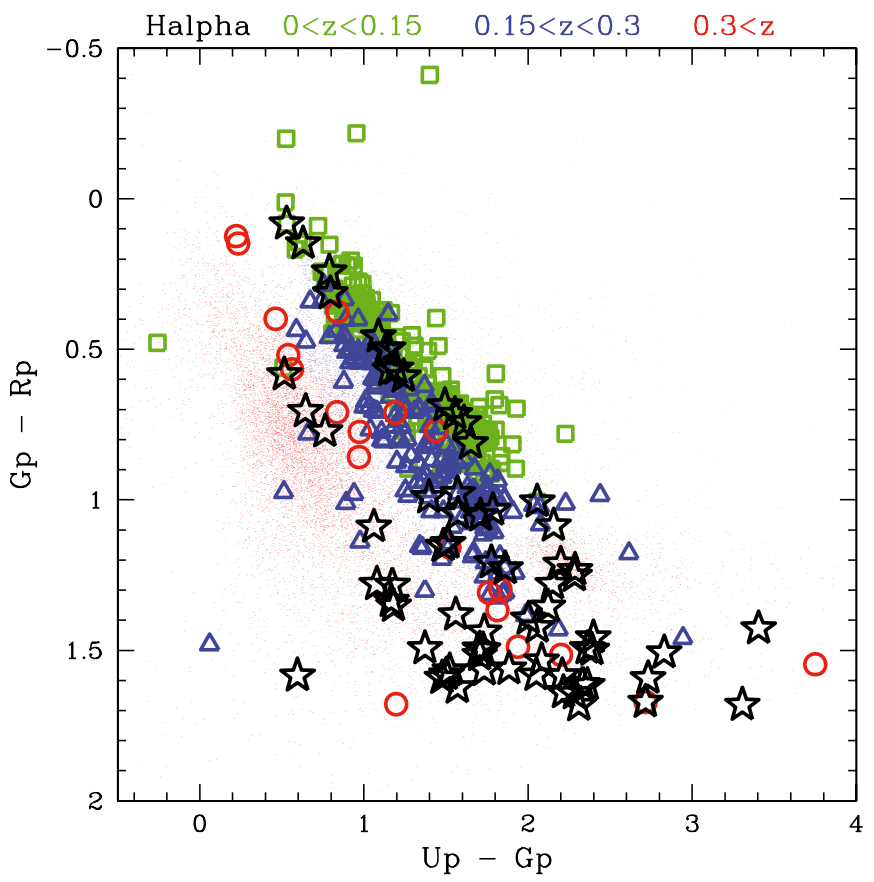

Fig. 13. Colour-colour diagramme for galaxies of known redshifts (green squares for $z<0.15$, blue triangles for $0.15<z<0.3$ and red circles for $z>0.3)$. The $\mathrm{H} \alpha$ detections are shown as black stars. Galaxies with only photometric redshifts are shown as green, blue, and red points, for $z_{\mathrm{p}}<0.5,0.15<z_{\mathrm{p}}<0.3$, and $z_{\mathrm{p}}>0.3$, respectively.

for the 58 galaxies detected in $\mathrm{H} \alpha$ displayed in Fig. 12 (top) shows a bimodal distribution, with many galaxies having photometric redshifts around $z_{\text {phot }} \sim 0.5$. This peak mainly corresponds to galaxies fainter than $R_{\mathrm{C}} \sim 19$ (see Fig. 12, middle), for which there are much fewer spectroscopic redshifts (the completeness of our full redshift catalogue drops to less than $50 \%$ for $R_{\mathrm{C}}>18$, see Table 4) and therefore for which photometric redshifts are less well "trained". A number of galaxies in this peak probably correspond to a population of rather faint galaxies at $z \sim 0.4$ emitting in $\mathrm{H} \beta$ or [OIII], while a few may in reality be at smaller distances and belong to Abell 85. Note that if these galaxies are all really at the distances corresponding to their photometric redshifts, the histogram of their absolute magnitudes (see Fig. 12, bottom) shows that most of them are intrinsically bright to very bright objects with absolute magnitudes between $M_{\mathrm{r}}=-22$ and -25 (absolute magnitudes were computed including $k$-correction, following Appendix A.1 of Ilbert et al. 2005). This would imply a high number of very intrinsically bright $(\approx 7 L *)$ galaxies in the field (they are spread throughout the WFI field so they do not seem to correspond to a background cluster). We are therefore inclined to believe that some of the $\mathrm{H} \alpha$ emitters that we detect and which have photometric redshifts larger than 0.2 may in reality be more nearby objects and could even possibly be members of Abell 85; the other objects are probably $\mathrm{H} \beta$ or [OIII] emitters at $z \sim 0.4$.

Figure 13 shows a colour-colour diagram for all the galaxies with measured redshifts. The $23 \mathrm{H} \alpha$ objects with redshifts in the cluster are those coinciding with the green squares.

In view of all these arguments, we will consider as detected in $\mathrm{H} \alpha$ and members of the Abell 85 cluster the 23 galaxies with spectroscopic redshifts in the [0.0451-0.0723] cluster range (plus galaxy ACO85J004127.86-092329.54 from the SDSS). We will also present our data on the 76 other galaxies detected in the 


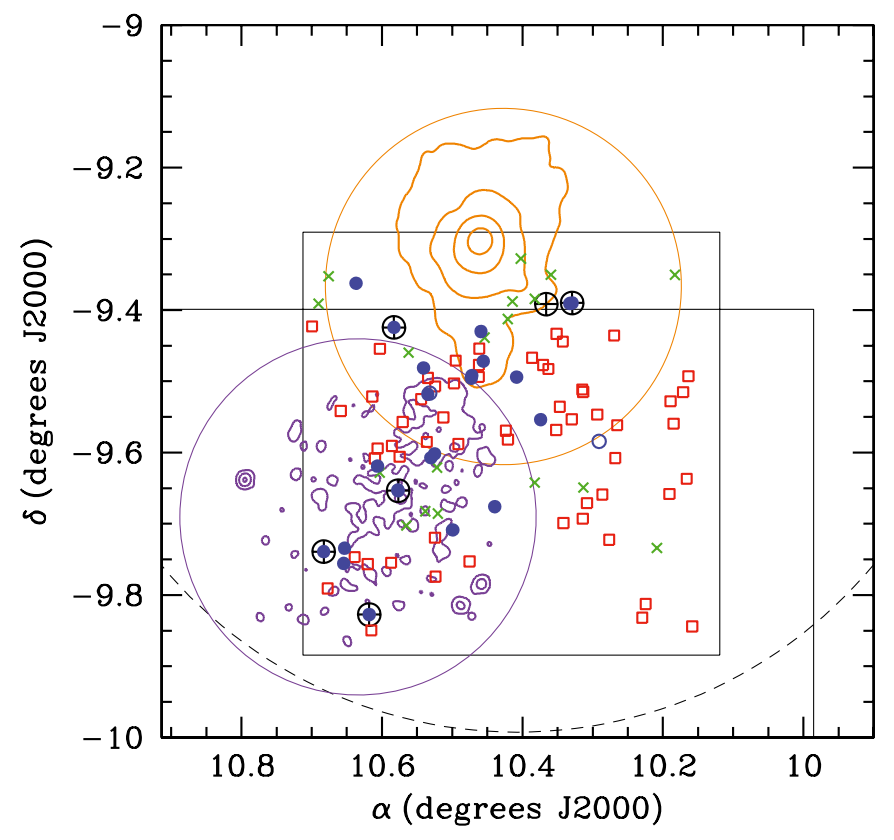

Fig. 14. Same as Fig. 4 for the 101 galaxies detected in the $\mathrm{H} \alpha$ filter, plus the SDSS galaxy. The blue circles show our $\mathrm{H} \alpha$ detections for galaxies with spectroscopic redshifts that place them in (filled circles) or out (open circles) of the cluster. Galaxies that we detect in $\mathrm{H} \alpha$ without spectroscopic redshifts are shown as red open squares or green crosses, depending on whether they have photometric redshifts (always at $z>0.2$ ) or no photometric redshifts, respectively. The galaxies detected in $\mathrm{H} \alpha$ by the SDSS are shown as large circles with plus sign signs (in particular ACO85J004127.86-092329.54, which we did not detect in $\mathrm{H} \alpha$ ).

$\mathrm{H} \alpha$ filter, keeping in mind that a good fraction of them is unlikely to belong to the cluster.

The full catalogues of these 101 galaxies are given in Tables 1-4 of the Appendix, together with postage stamp images of each galaxy in the $I^{\prime}, u^{*}, R_{\mathrm{C}}$ and "pure" $\mathrm{H} \alpha$ bands. Note that we added to Table 1 of the Appendix the SDSS galaxy ACO85J004127.86-092329.54.

\subsection{H $\alpha$ characteristics of the emission line galaxies}

From the fluxes measured by SExtractor in the $\mathrm{H} \alpha$ and $R_{\mathrm{C}}$ subimages for each detected galaxy, as described in Sect. 3.1, we now compute net $\mathrm{H} \alpha$ fluxes. The overlap of the $\mathrm{H} \alpha$ and $R_{\mathrm{C}}$ bands makes $\mathrm{H} \alpha$ net (i.e. continuum subtracted) flux calculations not straightforward. Let $C_{\mathrm{H} \alpha}$ and $C_{R_{\mathrm{C}}}$ be the counts per unit time measured in the $\mathrm{H} \alpha$ and $R_{\mathrm{C}}$ bands respectively. We can split these counts into two contributions: (1) the continuum $C_{\text {cont, } \mathrm{H} \alpha}$ and $C_{\text {cont, } R_{\mathrm{C}}}$ respectively and (2) the emission line $C_{\text {line }}$ corrected for the transmission factor of each filter:

$\begin{aligned} C_{\mathrm{H} \alpha} & =C_{\text {cont } \mathrm{H} \alpha}+T_{\mathrm{H} \alpha}\left(\lambda_{i}\right) C_{\text {line }}, \\ C_{R_{\mathrm{C}}} & =C_{\text {cont }, R_{\mathrm{C}}}+T_{R_{\mathrm{C}}}\left(\lambda_{i}\right) C_{\text {line }} .\end{aligned}$

Where $\lambda_{i}$ is the wavelength of the $\mathrm{H} \alpha$ emission line redshifted to the redshift of the $i$ th galaxy, and $T_{\mathrm{H} \alpha}(\lambda)$ and $T_{R_{\mathrm{C}}}(\lambda)$ are the transmission factors of the two filters. $C_{\mathrm{cont}, \mathrm{H} \alpha}$ and $C_{\mathrm{cont}, R_{\mathrm{C}}}$ are related to each other by

$\begin{aligned} C_{\text {cont }, \mathrm{H} \alpha} & =H \int T_{\mathrm{H} \alpha}(\lambda) \mathrm{d} \lambda, \\ C_{\text {cont }, R_{\mathrm{C}}} & =H \int T_{R_{\mathrm{C}}}(\lambda) \mathrm{d} \lambda\end{aligned}$ where $H$ is the height of the continuum (assumed to be constant). We can define the ratio between the integrated $R_{\mathrm{C}}$ and $\mathrm{H} \alpha$ filter transmissions with

$$
n=\frac{C_{\text {cont }, \mathrm{H} \alpha}}{C_{\text {cont }, R_{\mathrm{C}}}}=\frac{\int T_{\mathrm{H} \alpha}(\lambda) \mathrm{d} \lambda}{\int T_{R_{\mathrm{C}}}(\lambda) \mathrm{d} \lambda} .
$$

From Eqs. (1)-(4) we obtain the integrated $\mathrm{H} \alpha$ flux $F$ (in erg cm${ }^{-2} \mathrm{~s}^{-1}$ ) and the $\mathrm{H} \alpha$ equivalent width $E W$ (in $\AA$ ):

$$
\begin{aligned}
F & =\mathrm{ZP} \times C_{\text {line }}=\mathrm{ZP} \frac{C_{\mathrm{H} \alpha}-n C_{R_{\mathrm{C}}}}{T_{\mathrm{H} \alpha}\left(\lambda_{i}\right)-n T_{R_{\mathrm{C}}}\left(\lambda_{i}\right)}, \\
E W & =\frac{C_{\text {line }}}{H}=\frac{C_{\mathrm{H} \alpha}-n C_{R_{\mathrm{C}}}}{T_{\mathrm{H} \alpha}\left(\lambda_{i}\right) C_{R_{\mathrm{C}}}-T_{R_{\mathrm{C}}}\left(\lambda_{i}\right) C_{\mathrm{H} \alpha}} \int T_{R_{\mathrm{C}}}(\lambda) \mathrm{d} \lambda,
\end{aligned}
$$

where $\mathrm{ZP}$ is the zero point in $\mathrm{erg} \mathrm{cm}^{-2}$. The results are comparable but not identical to the equations of Boselli et al. (2002) and Gavazzi et al. (2002). $\mathrm{H} \alpha$ fluxes and equivalent widths are given for all the objects of our sample in Tables A.1-A.2 of the Appendix.

Since our catalogue contains galaxies without redshifts, we decided to correct the fluxes by constant factors $T_{\mathrm{H} \alpha}\left(\lambda_{i}\right)=0.9$ and $T_{R_{\mathrm{C}}}\left(\lambda_{i}\right)=0.9$. Thus, $\mathrm{H} \alpha$ luminosities and consequent star formation rates were computed from $\mathrm{H} \alpha$ fluxes assuming that all the galaxies are at the cluster redshift.

\subsection{Spatial distribution of the emission line galaxies}

The spatial distribution of the 101 galaxies detected in $\mathrm{H} \alpha$ and of the SDSS galaxy ACO85J004127.86-092329.54 is displayed in Fig. 14. As seen in this figure, most of the galaxies with redshifts in the cluster appear to be concentrated either in the periphery of the cluster, including the south blob (the group seen in $\mathrm{X}$-rays just south of the main cluster at coordinates $\alpha \sim 10.45^{\circ}$, $\delta \sim-9.45^{\circ}$ ) and along the filament. If we consider that the filament corresponds to the south east zone at $\alpha>10.45^{\circ}$ and $\delta<-9.5^{\circ}$, we can note that only 3 objects fall west of the filament in this southern area. We have seen when describing the galaxy luminosity functions in Sect. 4 that the Southeast part of the cluster has many more galaxies than the Southwest. Our hope when making these narrow band filter observations was to detect a high number of $\mathrm{H} \alpha$ emitters in the filament region, where star formation could have been enhanced by the movement of groups towards the cluster. Although more spectroscopically confirmed cluster members would be needed to claim such a result, it seems that $\mathrm{H} \alpha$ emitters in the cluster redshift range are more concentrated along the filament.

\subsection{Star formation in the filament region of Abell 85}

From the $\mathrm{H} \alpha$ fluxes, we estimated the $\mathrm{H} \alpha$ luminosities $L(\mathrm{H} \alpha)$ and, following Eq. (2) in Kennicutt (1998), the star formation rates (SFR) in the galaxies detected in the $\mathrm{H} \alpha$ filter: $\operatorname{SFR}\left(M_{\odot} \mathrm{yr}^{-1}\right)=7.9 \times 10^{-42} L(\mathrm{H} \alpha)$ (with $L(\mathrm{H} \alpha)$ in $\left.\mathrm{erg} \mathrm{s}^{-1}\right)$. Luminosities are estimated assuming that the galaxies belong to the cluster; they are obviously underestimated for background objects (for which it is not $\mathrm{H} \alpha$ emission that we are measuring but $\mathrm{H} \beta$ or [OIII]). Note that, due to the filter cut for galaxies with redshifts smaller than about 0.053 , star formation rates for objects with redshifts towards the lower limit of the cluster range are only lower limits.

$\mathrm{H} \alpha$ luminosities and star formation rates are given in Table A.1 of the Appendix. We can see that star formation rates are very small, smaller than $0.04 M_{\odot} / \mathrm{yr}$ except for one 
object having $0.24 M_{\odot} /$ yr. These values are comparable to or lower than those found e.g. by James et al. (2004) in a large sample of nearby galaxies and by Cortese et al. (2006) in the starbursting group falling into Abell 1367.

We have looked for variations of the $\mathrm{H} \alpha$ equivalent width along the direction of the filament but did not find any obvious trend.

An important caveat is that corresponding to completeness. As discussed in Sect. 2, the narrow band filter and a strong telluric absorption line make detections difficult for galaxies at redshifts lower than about 0.0515 . Therefore our $\mathrm{H} \alpha$ imaging is probably missing galaxies towards the lower range of the cluster redshift. Note also that the SDSS spectroscopic survey is probably not complete either.

\section{Discussion}

\subsection{Very large scale structure}

The infall directions are well known for Abell 85 (Durret et al. 1998 b, 2005). A previous merger is likely to have occured 3-4 Gyr ago from the Northwest, as derived from the presence of an "arc" of hotter X-ray gas regions, infall from the Southeast is presently been observed along the filament, and there is a third possible infall direction from the Northeast. The filament coming from the Southeast is obviously quite massive since it is detected in X-rays; from its X-ray temperature, it was suggested that it is probably made of groups falling on to the cluster (Durret et al. 2003). This agrees with our map of LFs showing very populated subfields towards the Southeast direction (see Figs. 7 and 8) and with our analysis based on the $\mathrm{H} \alpha$ image and on the $\mathrm{SG}$ analysis.

The distribution of groups and clusters with redshifts within \pm 0.005 (i.e. $\pm 1500 \mathrm{~km} \mathrm{~s}^{-1}$ ) of that of Abell 85 (extracted from the NED database) at very large scale is displayed in Fig. 15. Close to the cluster, a concentration of structures along a roughly North-Northwest to South-Southeast direction is observed. Further away, a concentration of structures towards the East-Southeast is seen, as well as two clusters even further away towards the Northeast and Southeast. Such a large concentration of clusters at redshifts very close to that of Abell 85 agrees with the general idea that this cluster has undergone and is still undergoing several merging events. It also indicates that there may be even more interactions going on than previously believed, and that although one of the preferential regions for merging activity is indicated by the filament, it may not be the only one.

\subsection{Are galaxies in the filament of Abell 85 more prone to bursts of star formation?}

We first note that our images extend to 2 virial radii, hence include the virialized part of the cluster, including the X-ray filament, plus a mainly infalling region beyond. The sampled regions are therefore populated with galaxies that are just beginning to undergo interactions with dense regions (the cluster itself or the dense parts of the filament as seen in X-rays). The faintend of the overall LF of these fields could be somewhat steeper in the $u^{*}$ band than in the other bands, but the error bar on the slope is large.

Our $\mathrm{H} \alpha$ detections may be preferentially concentrated in the filament and in the impact region, but the statistics are too low to draw a firm conclusion. Note besides that we do not detect any $\mathrm{H} \alpha$ filamentary structures (such as those found by

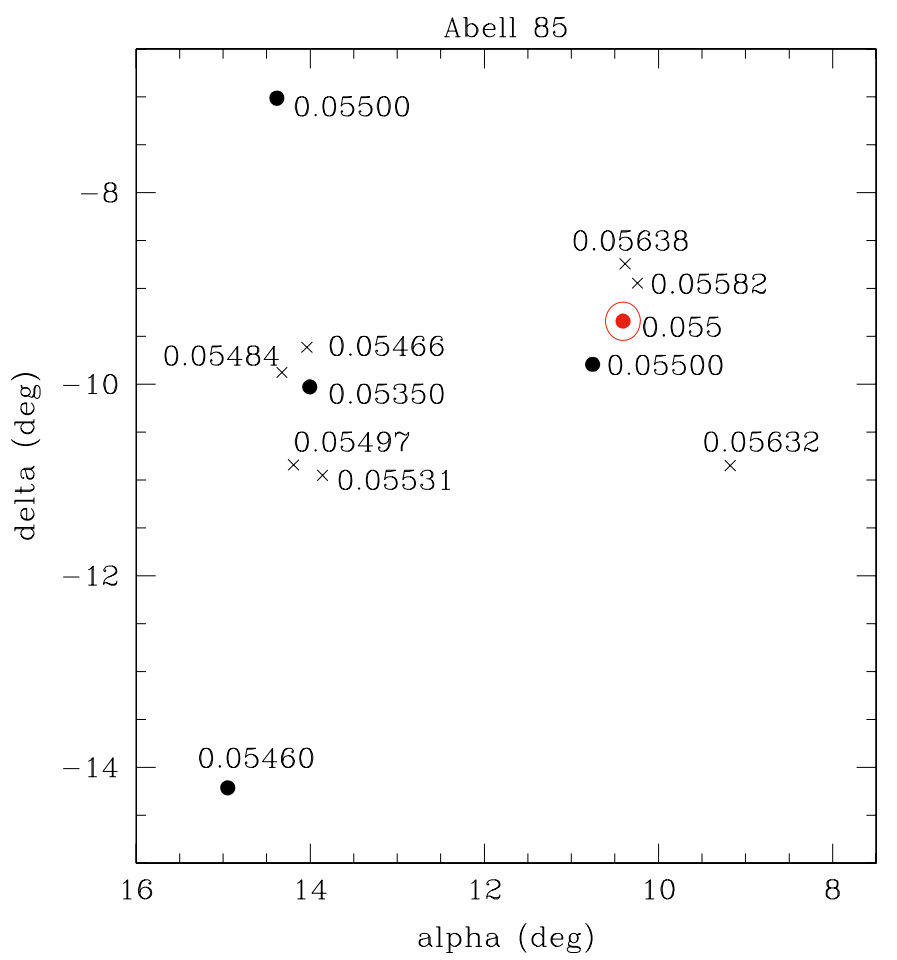

Fig. 15. Large scale structure surrounding Abell 85 taken from NED. The red circle shows the position of the cluster center, black circles are Abell clusters and crosses are other clusters found in the SDSS. Clusters and groups were selected to have a redshift within \pm 0.005 of that of the cluster, in a region of $8.5 \times 8.5 \mathrm{deg}^{2}\left(32 \times 32 \mathrm{Mpc}^{2}\right)$. Abell 85 is not exactly at the center of the figure because there are no groups or clusters towards the West and North of the cluster.

Cortese et al. 2006) which could be in the wake of galaxies having crossed the cluster.

The filament of Abell 85, detected in X-rays and consistent with the present optical data is composed of galaxies and gas. Since the gas temperature was found to be $2 \pm 1 \mathrm{keV}$, it cannot be identified with the cooler filaments predicted by numerical simulations of large scale structures (e.g. Davé et al. 2001). This agrees with our previous interpretation that the Abell 85 filament is made of groups.

\section{Conclusions}

We have analyzed an $\mathrm{H} \alpha$ image and broad band images covering the South of Abell 85 and its filament, which was previously discovered in X-rays (Durret et al. 1998b). The Galaxy Luminosity Functions in the South area of the cluster Abell 85 (including the impact region, where the groups constituting the filament hit the main cluster) and in the filament show the existence of a rich population of galaxies. The overall galaxy distribution in A85 is flattened with a principal axis that is parallel to the axis separating the filament seen in X-rays with the cluster center. All our results are consistent with the previous interpretation of the filament being made of groups falling onto the main cluster.

Acknowledgements. The authors are very grateful to the CFHT and Terapix teams for their efficiency in the Megacam data reduction, and to E. Bertin for his help in reducing the WFI data. We thank M. Montessuit for her contribution to the photometric calibration of the WFI data, I. Chilingarian for his twilight spectrum, and A. Boselli and R. Demarco for discussions. We also thank Matthew Colless for permission to use 6dFGS-DR3, in advance of publication. We acknowledge financial support from CNRS through the PNG and INSU, and from OPTICON. 


\section{References}

Adami, C., Marcelin, M., Amram, P., \& Russeil, D. 1999, A\&A, 349, 812 Adami, C., Biviano, A., Durret, F., \& Mazure, A. 2005, A\&A, 443, 17

Adami, C., Durret, F., Mazure, A., et al. 2007, A\&A, 462, 411

Bekki, K. 1999, ApJ, 510, L15

Bertin, E. 2006, SExtractor User's Manual,

http: //terapix.iap.fr/IMG/pdf/sextractor.pdf

Bertin, E., \& Arnouts, S. 1996, A\&AS, 117, 393

Blanton, M. R., Hogg, D. W., Bahcall, N. A., et al. 2003, ApJ, 592, 819

Boselli, A., Iglesias-Páramo, J., Vílchez, J. M., \& Gavazzi, G. 2002, A\&A, 386, 134

Boué, G., Adami, C., Durret, F., Mamon, G. A., \& Cayatte, V. 2008, A\&A, 479, 335

Bravo-Alfaro, H., Caretta, C., Lobo, C., Durret, F., \& Scott, T. 2008, A\&A, submitted

Cortese, L., Gavazzi, G., Boselli, A., \& Iglesias-Paramo, J. 2004, A\&A, 416, 119

Cortese, L., Gavazzi, G., Boselli, A., et al. 2006, A\&A, 453, 847

Crawford, C. S., Sanders, J. S., \& Fabian, A. C. 2005, MNRAS, 361, 17

Davé, R., Cen, R., Ostriker, J. P., et al. 2001, ApJ, 552, 473

Dressler, A. 1980, ApJ, 236, 351

Dressler, A., Oemler, A. J., Couch, W. J., et al. 1997, ApJ, 490, 577

Durret, F., Felenbok, P., Lobo, C., \& Slezak, E. 1998a, A\&AS, 129, 281

Durret, F., Forman, W., Gerbal, D., Jones, C., \& Vikhlinin, A. 1998b, A\&A, 335, 41

Durret, F., Lima Neto, G. B., Forman, W., \& Churazov, E. 2003, A\&A, 403, L29
Durret, F., Lima Neto, G. B., \& Forman, W. 2005, A\&A, 432, 809 Fukugita, M., Shimasaku, K., \& Ichikawa, T. 1995, PASP, 107, 945 Gavazzi, G., Boselli, A., Pedotti, P., Gallazzi, A., \& Carrasco, L. 2002, A\&A, 386,114

Gavazzi, G., Boselli, A., Cortese, L., et al. 2006, A\&A, 446, 839 Iglesias-Páramo, J., Boselli, A., Cortese, L., Vílchez, J. M., \& Gavazzi, G. 2002, A\&A, 384, 383

Ilbert, O., Tresse, L., Zucca, E., et al. 2005, A\&A, 439, 863

James, P. A., Shane, N. S., Beckman, J. E., et al. 2004, A\&A, 414, 23

Kennicutt, Jr., R. C. 1998, ARA\&A, 36, 189

Landolt, A. U. 1992, AJ, 104, 340

Moss, C., \& Whittle, M. 1993, ApJ, 407, L17

Moss, C., \& Whittle, M. 2000, MNRAS, 317, 667

Moss, C., \& Whittle, M. 2005, MNRAS, 357, 1337

Moss, C., Irwin, M. J., \& Whittle, M. 1988, MNRAS, 232, 381

Moss, C., Whittle, M., \& Pesce, J. E. 1998, MNRAS, 300, 205

Murayama, T., Taniguchi, Y., Scoville, N. Z., et al. 2007, ApJS, 172, 523

Pimbblet, K. A. 2005, MNRAS, 358, 256

Robin, A. C., Reylé, C., Derrière, S., \& Picaud, S. 2003, A\&A, 409, 523

Serna, A., \& Gerbal, D. 1996, A\&A, 309, 65

Springel, V., White, S. D. M., Jenkins, A., et al. 2005, Nature, 435, 629

Sun, M., Donahue, M., \& Voit, G. M. 2007, ApJ, 671, 190

Takahashi, M. I., Shioya, Y., Taniguchi, Y., et al. 2007, ApJS, 172, 456

Umeda, K., Yagi, M., Yamada, S. F., et al. 2004, ApJ, 601, 805

Yagi, M., Komiyama, Y., Yoshida, M., et al. 2007, ApJ, 660, 1209

Zucca, E., Ilbert, O., Bardelli, S., et al. 2006, A\&A, 455, 879 


\section{Appendix A:}

A full catalogue of the 101 galaxies detected in $\mathrm{H} \alpha$ is given Tables A.1-A.4 of the present Appendix, together with the galaxy ACO85J004127.86-092329.54 not detected in our $\mathrm{H} \alpha$ image due to the filter cut but with $\mathrm{H} \alpha$ emission in its SDSS spectrum (in Table A.1).

Tables A.1-A.3 respectively correspond to galaxies with spectroscopic redshifts, galaxies with photometric redshifts, and galaxies with no redshift information. They include the following columns: (1) number, (2) full IAU name, (3) spectroscopic redshift taken from NED, (4) $\mathrm{H} \alpha$ flux in erg cm$~^{-2} \mathrm{~s}^{-1}$ and error measured in the aperture in which the detection is made in the $R_{\mathrm{C}}$ band, (5) $\mathrm{H} \alpha$ equivalent width and error in $\AA$, (6) $\mathrm{H} \alpha$ luminosity in in erg s ${ }^{-1}$, (7) star formation rate in $M_{\odot} / \mathrm{yr}$, (8) factor $n$ by which the $R_{\mathrm{C}}$ image was multiplied before being subtracted from the $\mathrm{H} \alpha$ image.

Tables 4 and 5 give: (1) number, (2) full IAU name, (3)-(7) $R_{\mathrm{C}}, r^{\prime}, u^{*}, g^{\prime}$ and $i^{\prime}$ band magnitudes (SExtractor MAG_AUTO).

Images are displayed for each galaxy in the $i^{\prime}, u^{*}, R_{\mathrm{C}}$ and "pure" $\mathrm{H} \alpha$ bands in Figs. 1-101. Since the Megacam image does not fully cover the WFI image, some galaxies have no Megacam $\left(u^{*}\right.$ and $\left.i^{\prime}\right)$ data.

The spectra of the 6 SDSS galaxies with $E W(\mathrm{H} \alpha)>3 \AA$ are displayed in Figs. 102-107. 
G. Boué et al.: Filament of Abell 85, Online Material p 2

Table A.1. Catalogue of the 25 galaxies detected in $\mathrm{H} \alpha$ and with spectroscopic redshifts (the first 23 belong to the cluster), also including the SDSS galaxy ACO85J004127.86-092329.54.

\begin{tabular}{|c|c|c|c|c|c|c|c|}
\hline$\overline{\text { Num }}$ & $\overline{\text { Name }}$ & $z_{\text {spec }}$ & 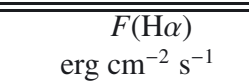 & $\begin{array}{c}E W(\mathrm{H} \alpha) \\
\AA\end{array}$ & $\begin{array}{l}L(\mathrm{H} \alpha) \\
\mathrm{erg} \mathrm{s}^{-1}\end{array}$ & $\begin{array}{r}S F R \\
M_{\odot} \mathrm{yr}^{-1}\end{array}$ & $n$ \\
\hline 24 & ACO85J004119.01-092323.5 & 0.0498 & $1.19 \mathrm{e}-16 \pm 7.2 \mathrm{e}-18$ & $13.3 \pm 0.8$ & $8.32 \mathrm{e}+38$ & 0.00658 & 0.118765 \\
\hline 26 & ACO85J004119.83-092327.0 & 0.0490 & $1.16 \mathrm{e}-16 \pm 7.8 \mathrm{e}-18$ & $4.2 \pm 0.3$ & $8.15 e+38$ & 0.00644 & 0.118765 \\
\hline 35 & ACO85J004129.77-093313.2 & 0.0624 & $4.13 e-16 \pm 9.3 e-18$ & $129.3 \pm 3.3$ & $2.90 \mathrm{e}+39$ & 0.02288 & 0.115607 \\
\hline 40 & ACO85J004138.01-092938.0 & 0.0497 & $6.91 \mathrm{e}-17 \pm 3.8 \mathrm{e}-18$ & $13.9 \pm 0.8$ & $4.85 e+38$ & 0.00383 & 0.116822 \\
\hline 45 & ACO85J004145.45-094033.1 & 0.0569 & $2.33 e-16 \pm 1.0 e-17$ & $17.2 \pm 0.7$ & $1.64 \mathrm{e}+39$ & 0.01294 & 0.11534 \\
\hline 47 & ACO85J004149.38-092818.3 & 0.0723 & $1.07 \mathrm{e}-16 \pm 5.7 \mathrm{e}-18$ & $20.4 \pm 1.1$ & $7.49 e+38$ & 0.00592 & 0.117371 \\
\hline 48 & ACO85J004150.17-092547.6 & 0.0579 & $7.60 \mathrm{e}-17 \pm 1.3 \mathrm{e}-17$ & $1.7 \pm 0.3$ & $5.34 \mathrm{e}+38$ & 0.00422 & 0.118765 \\
\hline 52 & ACO85J004153.27-092930.4 & 0.0525 & $4.35 e-15 \pm 3.8 e-17$ & $47.9 \pm 0.5$ & $3.05 e+40$ & 0.24093 & 116822 \\
\hline 53 & ACO85J004153.51-092943.8 & 0.0510 & $e-17 \pm 3.1 e-17$ & $3.1 \pm 1.1$ & $6.13 e+38$ & 0.00485 & 16686 \\
\hline 58 & 004159.84-094230.9 & & 18 & $15.8 \pm 1.6$ & $6.79 e+38$ & 0.00537 & 16009 \\
\hline 65 & $.02-09$ & 0.0 & $e-18$ & $5.7 \pm 0.5$ & $4.71 e+38$ & 0.00372 & 0.114943 \\
\hline 66 & $\mathrm{AC}$ & & 7 & $49.1 \pm 1.1$ & $3.76 e+39$ & 972 & 4943 \\
\hline 69 & $36-093$ & 0.0 & $7 \pm 7$ & $15.0 \pm 1.3$ & $6.55 e+38$ & 517 & 6009 \\
\hline 72 & $31-092$ & 0.0 & $6 \pm 8$ & $54.6 \pm 0.8$ & $4.39 \mathrm{e}+39$ & 0.03471 & 6959 \\
\hline 78 & $.47-093$ & 0.0515 & $6 \pm 1.5 \mathrm{e}-17$ & $39.3 \pm 0.9$ & $4.93 e+39$ & 0.03893 & 15207 \\
\hline 79 & $3.55-093910.2$ & 0.0515 & $16 \pm 1.4 \mathrm{e}-17$ & $59.4 \pm 1.6$ & $3.75 e+39$ & 0.02964 & 0.115207 \\
\hline 80 & $.89-092527.5$ & 0.0512 & $16 \pm 5.1 \mathrm{e}-18$ & $17.0 \pm 0.5$ & $19 e+39$ & 0.00939 & 0.118064 \\
\hline 86 & $.54-09$ & 0.0 & $7 \pm 3.7 \mathrm{e}-18$ & $6.6 \pm 0.7$ & $2.50 \mathrm{e}+38$ & 0.00198 & 0.115075 \\
\hline 90 & $38-094938.3$ & 0.0501 & $17 \pm 6.5 \mathrm{e}-18$ & $9.8 \pm 0.8$ & $5.58 \mathrm{e}+38$ & 0.00441 & 0.116959 \\
\hline 92 & $84-092144.2$ & 59 & $17 \pm 7.2 \mathrm{e}-18$ & $11.9 \pm 1.1$ & $5.38 \mathrm{e}+38$ & 0.00425 & 0.117509 \\
\hline 94 & $36.76-094403.8$ & 0.0643 & $17 \pm 6.0 \mathrm{e}-18$ & $16.0 \pm 1.0$ & $7.01 e+38$ & 0.00554 & 0.115741 \\
\hline 95 & ACO85J004237.07-094520.5 & 0.0569 & $5.74 \mathrm{e}-17 \pm 6.2 \mathrm{e}-18$ & $7.6 \pm 0.8$ & $4.03 e+38$ & 0.00318 & 0.115875 \\
\hline \multirow[t]{2}{*}{99} & ACO85J004243.90-094420.8 & 0.0508 & $1.20 \mathrm{e}-16 \pm 9.4 \mathrm{e}-18$ & $10.8 \pm 0.9$ & $8.44 e+38$ & 0.00667 & 0.114943 \\
\hline & ACO85J004127.86-092329.54 & 0.0494 & & $4.5 \pm 0.3$ & & & \\
\hline 17 & ACO85J004109.80-093503.0 & 0.2331 & $5.23 e-17 \pm 5.8 \mathrm{e}-18$ & $7.8 \pm 0.9$ & $3.67 e+38$ & 0.00290 & 0.114811 \\
\hline 67 & ACO85J004207.71-093059.3 & 0.4355 & $2.86 \mathrm{e}-16 \pm 1.4 \mathrm{e}-17$ & $37.7 \pm 1.9$ & $2.00 \mathrm{e}+39$ & 0.01583 & 0.116009 \\
\hline
\end{tabular}


G. Boué et al.: Filament of Abell 85, Online Material p 3

Table A.2. Catalogue of the 58 galaxies detected in $\mathrm{H} \alpha$ and with photometric redshifts.

\begin{tabular}{|c|c|c|c|c|c|c|c|c|c|}
\hline Num & Name & $z_{\text {phot }}$ & $z_{\text {inf }}$ & $z_{\text {sup }}$ & 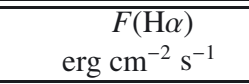 & $\begin{array}{c}E W(\mathrm{H} \alpha) \\
\AA\end{array}$ & $\begin{array}{l}L(\mathrm{H} \alpha) \\
\mathrm{erg} \mathrm{s}^{-1} \\
\end{array}$ & $\begin{array}{r}S F R \\
M_{\odot} \mathrm{yr}^{-1} \\
\end{array}$ & $n$ \\
\hline 1 & ACO85J004037.99-095038.8 & 0.5571 & 0.5300 & .5800 & $5.63 e-17 \pm 6.4 e-18$ & $75.1 \pm 9.0$ & $3.95 e+38$ & 0.00312 & 0.0986193 \\
\hline 2 & ACO85J004039.27-092933.8 & 6215 & 6000 & 5500 & $7.98 \mathrm{e}-17 \pm 5.8 \mathrm{e}-18$ & $60.1 \pm 4.6$ & $5.60 e+38$ & 0.00442 & 08814 \\
\hline 3 & ACO85J004039.90-093812.5 & 0.4628 & 4400 & 4800 & $7.19 \mathrm{e}-17 \pm 6.8 \mathrm{e}-18$ & $23.5 \pm 2.3$ & $5.05 e+38$ & 00399 & 08225 \\
\hline 4 & ACO85J004041.11-093054.7 & 0.6063 & 5800 & 6300 & $6.90 \mathrm{e}-17 \pm 7.1 \mathrm{e}-18$ & $40.0 \pm 4.3$ & $4.84 e+38$ & 00383 & 109649 \\
\hline 6 & ACO85J004044.33-093334.4 & 0.6150 & 0.5900 & 6500 & $1.04 \mathrm{e}-16 \pm 7.5 \mathrm{e}-18$ & $55.7 \pm 4.3$ & $7.27 \mathrm{e}+38$ & 00574 & 10619 \\
\hline 7 & ACO85J0040 & & 4700 & 5300 & $2 e-18$ & $27.0 \pm 2.9$ & $4.11 e+38$ & 00325 & \\
\hline 8 & $\mathrm{ACC}$ & & 0 & 00 & 8 & $.4 \pm 11.9$ & $6 e+38$ & 00313 & \\
\hline 10 & $\mathrm{AC}$ & & & & 8 & $121.3 \pm 13.5$ & $5.23 e+38$ & 413 & \\
\hline 11 & $\mathrm{ACO}$ & & 00 & 00 & $17 \pm$ & \pm 4.3 & $5.26 e+38$ & 00416 & 08578 \\
\hline 12 & $\mathrm{ACC}$ & & 000 & 3600 & $17 \pm$ & \pm 7.2 & $83 e+38$ & 0223 & 14548 \\
\hline 13 & $\mathrm{ACC}$ & & 200 & 7700 & $17 \pm$ & $3 \pm 3.4$ & $3.65 e+38$ & 288 & \\
\hline 14 & $67-092$ & & 4400 & 5700 & 5.7 & \pm 2.5 & $4.01 e+38$ & 00317 & 279 \\
\hline 15 & 004106.35-094321.1 & 31 & .4900 & 5400 & $7.39 \mathrm{e}-17 \pm$ & + \pm 3.8 & $5.19 e+38$ & 00410 & 14025 \\
\hline 16 & J004108.66-093932.2 & 0.4685 & 0.4500 & 4900 & $4.27 e-17 \pm$ & \pm 3.2 & $3.00 \mathrm{e}+38$ & .00237 & 0.114416 \\
\hline 18 & $004110.45-093247.7$ & 0.5440 & 0.5000 & 6000 & $8.15 e-17 \pm 5.7 e-18$ & \pm 5.4 & $5.72 e+38$ & .00452 & 0.11534 \\
\hline 19 & 96-094015.3 & 0.4717 & .4500 & 4900 & $6.64 \mathrm{e}-17 \pm$ & \pm 2.9 & $4.66 e+38$ & .00368 & \\
\hline 21 & $\mathrm{AC}$ & & & & & 12.1 & $3.20 \mathrm{e}+38$ & 0253 & \\
\hline 22 & & & & 3000 & 18 & \pm 0.7 & & 400 & \\
\hline 23 & & & & & & $=4.9$ & & 0573 & \\
\hline 25 & $\mathrm{AC}$ & & & & & \pm 1.8 & & 0441 & \\
\hline 27 & $\mathrm{ACC}$ & & & & & $8 \pm 14.0$ & & & \\
\hline 28 & $\mathrm{AC}$ & & & & 8 & 10.8 & 1.59 & 256 & \\
\hline 29 & $\mathrm{AC}$ & & 0 . & 0 & $7 \pm$ & \pm 7.3 & 4.44 & 351 & \\
\hline 30 & $\mathrm{AC}$ & & 0 & 0 & 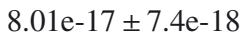 & \pm 3.1 & 38 & 444 & \\
\hline 31 & $\mathrm{AC}$ & & 00 & 0 & 3 & \pm 3.1 & 2.7 & 219 & \\
\hline 33 & $\mathrm{AC}$ & & 00 & 0 & $7=$ & \pm 6.1 & 38 & 304 & 33 \\
\hline 34 & $95-092$ & 0.9271 & .9100 & .9400 & $17 \pm$ & \pm 5.0 & $3.34 \mathrm{e}+38$ & 00264 & 371 \\
\hline 38 & $\mathrm{ACC}$ & 33 & 800 & 0 & $7 \pm$ & \pm 4.2 & $4.76 e+38$ & 376 & 7647 \\
\hline 42 & $\mathrm{AC}$ & 4 & 6000 & 6400 & $6 \pm$ & \pm 1.8 & $2.06 e+39$ & 1628 & 5207 \\
\hline 44 & $\mathrm{AC}$ & & 00 & 0 & 8 & $=2.4$ & 38 & 85 & 1534 \\
\hline 49 & $\mathrm{AC}$ & & 900 & 0 & 2. & 1.3 & 39 & & 25 \\
\hline 50 & $\mathrm{AC}$ & & 700 & & 6 & 5.3 & 4. & 67 & 33 \\
\hline 51 & & & & & & & & & \\
\hline 54 & $\mathrm{AC}$ & & & & 18 & 5.5 & 38 & 38 & 322 \\
\hline 55 & $\mathrm{AC}$ & & & & 18 & & & 85 & \\
\hline 56 & & & & & & & & 53 & \\
\hline 57 & $\mathrm{AC}$ & & & & 8 & & & & \\
\hline 59 & $\mathrm{AC}$ & & & & & $=3.6$ & 38 & 78 & \\
\hline 62 & $\mathrm{AC}$ & & & & 8 & $5 \pm 2.1$ & $5.11 \mathrm{e}$ & 403 & \\
\hline 63 & $\mathrm{ACC}$ & & 00 & 00 & $17 \pm$ & $29.2 \pm 2.6$ & $5.72 \mathrm{e}+38$ & 0.00452 & 279 \\
\hline 64 & $\mathrm{ACC}$ & 59 & 4600 & 00 & $16 \pm$ & $22.5 \pm 0.2$ & $3.84 \mathrm{e}+39$ & 0.03030 & 0.11 \\
\hline 68 & $\mathrm{ACC}$ & 8 & 0 & 0 & $17 \pm$ & $27.8 \pm 2.5$ & $5.92 \mathrm{e}+38$ & 0467 & 655 \\
\hline 70 & $\mathrm{ACC}$ & & 0 & 0 & $6+$ & \pm 4.4 & $1.02 \mathrm{e}+39$ & 304 & 75 \\
\hline 73 & $\mathrm{AC}$ & 6 & 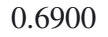 & 0 & $7-$ & \pm 5.7 & $4.77 e+38$ & 377 & 375 \\
\hline 76 & $\mathrm{AC}$ & 4148 & 0.3900 & 0 & 9 & \pm 3.1 & $6.82 \mathrm{e}+38$ & 39 & 34 \\
\hline 77 & $\mathrm{AC}$ & 8 & 0 & .7400 & 8 & \pm 4.9 & $6.07 e+38$ & 480 & 775 \\
\hline 81 & $\mathrm{AC}$ & & & & & 2.7 & +38 & 337 & 075 \\
\hline 82 & $\mathrm{ACO}$ & 0.7646 & 0.7300 & 0.8100 & 18 & $55.9 \pm 5.6$ & $5.27 e+38$ & 0.00416 & 6822 \\
\hline 83 & $\mathrm{ACO}$ & 0.4294 & 0.4000 & 0.4800 & -18 & 2.6 & $6.39 e+38$ & 0.00505 & 17233 \\
\hline 85 & $\mathrm{AC}$ & & & & & 4.5 & & 478 & 15075 \\
\hline 87 & $\mathrm{ACO}$ & & & & & $2 \pm 2.4$ & $4.70 e+38$ & 0.00371 & 075 \\
\hline 88 & $\mathrm{ACC}$ & & & & 6.7 & $27.4 \pm 1.4$ & $4.72 \mathrm{e}+38$ & 0.00373 & 115741 \\
\hline 89 & $\mathrm{ACC}$ & & & & 1.0 & $51.0 \pm 3.6$ & $7.33 e+38$ & 0.00579 & 0.116822 \\
\hline 91 & $\mathrm{ACO}$ & & & & $3.71 \mathrm{e}-17 \pm$ & $21.7 \pm 3.1$ & $2.60 e+38$ & 0.00206 & 0.11655 \\
\hline 93 & & & & & & 2.4 & $5.00 \mathrm{e}+38$ & 0.00395 & \\
\hline 96 & & & & & & & & .00448 & \\
\hline 98 & & & & & & & & & \\
\hline 101 & 5J004247.85-092522.6 & & & 00 & 6.50 & 14.8 & $4.56 e+38$ & 0.00361 & 0.114679 \\
\hline
\end{tabular}


Table A.3. Catalogue of the 18 galaxies detected in $\mathrm{H} \alpha$ and with no photometric redshifts.

\begin{tabular}{rlrrrrr}
\hline \hline Num & Name & $\begin{array}{r}F(\mathrm{H} \alpha) \\
\mathrm{erg} \mathrm{cm}^{-2} \mathrm{~s}^{-1}\end{array}$ & $\begin{array}{r}E W(\mathrm{H} \alpha) \\
\AA\end{array}$ & $\begin{array}{r}L(\mathrm{H} \alpha) \\
\mathrm{erg} \mathrm{s}^{-1}\end{array}$ & $\begin{array}{r}S F R \\
M_{\odot} \mathrm{yr}^{-1}\end{array}$ & $n$ \\
\hline 5 & ACO85J004043.91-092101.7 & $1.26 \mathrm{e}-16 \pm 4.7 \mathrm{e}-18$ & $34.3 \pm 1.3$ & $8.82 \mathrm{e}+38$ & 0.00697 & 0.107296 \\
9 & ACO85J004050.05-094402.5 & $6.24 \mathrm{e}-17 \pm 6.5 \mathrm{e}-18$ & $45.7 \pm 4.9$ & $4.38 \mathrm{e}+38$ & 0.00346 & 0.110132 \\
20 & ACO85J004115.20-093856.8 & $3.97 \mathrm{e}-17 \pm 4.3 \mathrm{e}-18$ & $20.6 \pm 2.3$ & $2.79 \mathrm{e}+38$ & 0.00220 & 0.114811 \\
32 & ACO85J004126.27-092101.5 & $1.02 \mathrm{e}-16 \pm 8.5 \mathrm{e}-18$ & $42.2 \pm 3.7$ & $7.17 \mathrm{e}+38$ & 0.00566 & 0.11976 \\
36 & ACO85J004131.77-093832.1 & $3.09 \mathrm{e}-16 \pm 9.6 \mathrm{e}-18$ & $55.1 \pm 1.8$ & $2.17 \mathrm{e}+39$ & 0.01714 & 0.115075 \\
37 & ACO85J004131.80-092303.7 & $6.66 \mathrm{e}-17 \pm 7.0 \mathrm{e}-18$ & $56.2 \pm 6.3$ & $4.67 \mathrm{e}+38$ & 0.00369 & 0.11976 \\
39 & ACO85J004136.55-091939.5 & $5.66 \mathrm{e}-17 \pm 8.9 \mathrm{e}-18$ & $14.5 \pm 2.3$ & $3.98 \mathrm{e}+38$ & 0.00314 & 0.120627 \\
41 & ACO85J004139.37-092316.3 & $6.85 \mathrm{e}-17 \pm 7.7 \mathrm{e}-18$ & $63.0 \pm 7.5$ & $4.81 \mathrm{e}+38$ & 0.00380 & 0.120048 \\
43 & ACO85J004141.04-092444.9 & $7.36 \mathrm{e}-17 \pm 7.7 \mathrm{e}-18$ & $57.0 \pm 6.3$ & $5.17 \mathrm{e}+38$ & 0.00408 & 0.119332 \\
46 & ACO85J004148.91-092618.7 & $7.45 \mathrm{e}-17 \pm 7.6 \mathrm{e}-18$ & $64.1 \pm 7.0$ & $5.23 \mathrm{e}+38$ & 0.00413 & 0.118483 \\
60 & ACO85J004204.90-094108.6 & $9.07 \mathrm{e}-17 \pm 7.1 \mathrm{e}-18$ & $24.4 \pm 2.0$ & $6.36 \mathrm{e}+38$ & 0.00503 & 0.115607 \\
61 & ACO85J004205.15-093715.5 & $2.39 \mathrm{e}-16 \pm 6.0 \mathrm{e}-18$ & $155.4 \pm 4.5$ & $1.68 \mathrm{e}+39$ & 0.01326 & 0.114943 \\
71 & ACO85J004209.19-094056.6 & $9.47 \mathrm{e}-17 \pm 8.0 \mathrm{e}-18$ & $57.1 \pm 5.1$ & $6.64 \mathrm{e}+38$ & 0.00525 & 0.115607 \\
74 & ACO85J004214.92-092735.4 & $5.99 \mathrm{e}-17 \pm 7.7 \mathrm{e}-18$ & $26.5 \pm 3.5$ & $4.20 \mathrm{e}+38$ & 0.00332 & 0.117371 \\
75 & ACO85J004215.64-094209.2 & $4.77 \mathrm{e}-17 \pm 6.7 \mathrm{e}-18$ & $34.7 \pm 5.0$ & $3.35 \mathrm{e}+38$ & 0.00265 & 0.115875 \\
84 & ACO85J004224.74-093741.3 & $3.37 \mathrm{e}-17 \pm 3.1 \mathrm{e}-18$ & $37.9 \pm 3.6$ & $2.36 \mathrm{e}+38$ & 0.00187 & 0.115075 \\
97 & ACO85J004242.24-092108.7 & $6.00 \mathrm{e}-17 \pm 6.7 \mathrm{e}-18$ & $105.8 \pm 13.0$ & $4.21 \mathrm{e}+38$ & 0.00333 & 0.11534 \\
100 & ACO85J004245.68-092327.8 & $4.90 \mathrm{e}-17 \pm 7.7 \mathrm{e}-18$ & $72.7 \pm 12.2$ & $3.44 \mathrm{e}+38$ & 0.00272 & 0.115075 \\
\hline
\end{tabular}

Table A.3 gives the list of 6 galaxies not detected in our $\mathrm{H} \alpha$ image due to the filter cut but with $\mathrm{H} \alpha$ emission in their SDSS spectrum. It includes the following columns: (1) number, (2) full IAU name, (3) SDSS spectroscopic redshift, (4) SDSS $r$ magnitude, (5) $\mathrm{H} \alpha$ equivalent width in $\AA$, (6) error on the $\mathrm{H} \alpha$ equivalent width in $\AA$. 
Table A.4. Broad band magnitudes of the 101 objects detected in $\mathrm{H} \alpha$.

\begin{tabular}{|c|c|c|c|c|c|c|}
\hline$\overline{\text { Num }}$ & Name & $R_{\mathrm{C}}$ & $r^{\prime}$ & $u^{*}$ & $g^{\prime}$ & $i^{\prime}$ \\
\hline 1 & ACO85J004037.99-095038.8 & 21.02 & 21.48 & 25.24 & 23.04 & 20.48 \\
\hline 2 & ACO85J004039.27-092933.8 & 20.65 & 21.08 & 24.80 & 22.36 & 19.48 \\
\hline 3 & ACO85J004039.90-093812.5 & 19.34 & 19.68 & 23.72 & 21.28 & 18.96 \\
\hline 4 & ACO85J004041.11-093054.7 & 20.04 & 20.58 & 24.41 & 22.14 & 19.48 \\
\hline 5 & ACO85J004043.91-092101.7 & 18.95 & - & - & - & 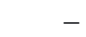 \\
\hline 6 & ACO85J004044.33-093334.4 & 20.08 & 20.60 & 24.41 & 22.23 & 9.57 \\
\hline 7 & ACO85J004045.38-093140.9 & 19.81 & 20.23 & 23.54 & 21.74 & 19.41 \\
\hline 8 & ACO85J004045.80-093929.1 & 21.17 & 21.82 & 24.51 & 23.27 & 20.37 \\
\hline 9 & $.05-09$ & 20.19 & 20.70 & 24.38 & 22.21 & 19.70 \\
\hline 10 & $.86-094845.6$ & 21.39 & 21.95 & 24.83 & 23.41 & 20.58 \\
\hline 11 & ACO85J004055.06-094954.9 & 20.13 & 20.53 & 24.26 & 22.08 & 19.72 \\
\hline 12 & ACO85J004103.65-093 & 21.30 & 22.04 & 25.34 & 23.28 & 20.22 \\
\hline 13 & ACO85J004104.27-093627.8 & 20.46 & 21.08 & 24.45 & 22.32 & 19.40 \\
\hline 14 & ACO85J004104.67-092608.6 & 19.66 & 19.92 & 22.88 & 21.23 & 19.22 \\
\hline 15 & ACO85J004106.35-094321.1 & 19.76 & 20.14 & 23.47 & 21.69 & 19.40 \\
\hline 16 & ACO85J004108.66-093932.2 & 20.37 & 20.88 & 24.18 & 22.32 & 20.03 \\
\hline 17 & ACO85J004109.80-093503.0 & 17.73 & 17.95 & 20.93 & 19.16 & 17.45 \\
\hline 18 & ACO85J004110.45-093247.7 & 20.68 & 20.75 & 23.38 & 22.11 & 19.81 \\
\hline 19 & ACO85J004113.96-094015.3 & 19.56 & 19.95 & 23.64 & 21.48 & 19.23 \\
\hline 20 & ACO85J004115.20-093856.8 & 19.74 & - & - & - & - \\
\hline 21 & ACO85J004115.27-093053.9 & 21.57 & 21.88 & 25.68 & 23.54 & 20.79 \\
\hline 22 & ACO85J004115.38-094134.6 & 18.02 & 18.37 & 21.09 & 19.43 & 17.86 \\
\hline 23 & $\mathrm{ACO}$ & 19.99 & 20.34 & 23.58 & 21.88 & 19.42 \\
\hline 24 & $\mathrm{CO}$ & 17.33 & - & - & - & - \\
\hline 25 & .0 & 19.08 & 19.17 & 23.03 & 20.77 & 18.48 \\
\hline 26 & $\mathrm{ACO}$ & 16.55 & - & - & - & - \\
\hline 27 & $\mathrm{ACO}$ & 21.26 & 21.52 & 24.10 & 22.87 & 20.29 \\
\hline 28 & $17-0$ & 21.26 & 21.70 & 22.28 & 21.98 & 21.54 \\
\hline 29 & $.20-093208.6$ & 20.68 & 20.98 & 26.28 & 22.60 & 19.88 \\
\hline 30 & 4.32-092600.2 & 19.76 & 19.94 & 23.42 & 21.48 & 19.17 \\
\hline 31 & ACO85J004124.48-093405.3 & 20.21 & 20.30 & 21.76 & 1.06 & 19.99 \\
\hline 32 & ACO85J004126.27-092101.5 & 19.67 & - & - & - & - \\
\hline 33 & 7.13-092 & 20.39 & 20.70 & 4.30 & 2.19 & 19.58 \\
\hline 34 & $.95-092837.1$ & 20.58 & 20.90 & 5.05 & 2.45 & 19.74 \\
\hline 35 & $.77-0$ & 19.21 & 19.33 & 19.94 & 19.38 & 19.10 \\
\hline 36 & 1 & 88 & - & - & - & -5 \\
\hline 37 & $\mathrm{ACC}$ & 26 & - & - & - & \\
\hline 38 & .7 & 86 & 20.11 & 23.14 & 21.56 & 19.06 \\
\hline 39 & & 95 & - & - & - & \\
\hline 40 & .0 & 50 & 8.58 & 20.10 & 19.03 & 18.28 \\
\hline 41 & $\mathrm{CC}$ & 20.87 & - & - & - & 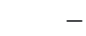 \\
\hline 42 & $92-0$ & 19.28 & 20.00 & 23.35 & 21.18 & 18.29 \\
\hline 43 & $1.04-092444.9$ & 20.56 & 20.94 & 24.27 & 22.45 & 19.96 \\
\hline 44 & $1.67-093409.5$ & 19.21 & 19.59 & 23.37 & 21.24 & 18.80 \\
\hline 45 & 5.45-094033.1 & 16.79 & 16.98 & 18.63 & 17.49 & 16.69 \\
\hline 46 & $.91-092$ & 20.85 & 21.37 & 25.03 & 2.95 & 20.29 \\
\hline 47 & $.38-092$ & 17.90 & 18.26 & 19.99 & 8.81 & 17.95 \\
\hline 48 & $17-09$ & 14.51 & 15.26 & 17.87 & 16.09 & 14.83 \\
\hline 49 & & & 19.63 & 22.44 & 20.69 & 18.34 \\
\hline 50 & $\mathrm{AC}$ & 20.49 & 21.00 & 25.14 & 22.57 & 19.82 \\
\hline 51 & ACO85J004150.94-092938.1 & 19.51 & 19.78 & 23.50 & 21.39 & 19.09 \\
\hline 52 & & & & 17.12 & 5.34 & 15.83 \\
\hline 53 & & & 15.27 & 17.53 & 15.97 & 14.88 \\
\hline 54 & .2 & 20.77 & 21.11 & 24.11 & 22.58 & 20.25 \\
\hline 55 & $\mathrm{CO} 8$ & 19.56 & 20.05 & 23.09 & 21.13 & 18.94 \\
\hline 56 & $8.81-092815.3$ & 20.17 & 20.56 & 23.82 & 21.98 & 19.60 \\
\hline 57 & $9.36-093010.9$ & 20.13 & 20.56 & 24.35 & 22.14 & 19.77 \\
\hline 5 & $.84-094$ & 17.36 & 17.58 & 18.83 & 17.92 & 17.41 \\
\hline 5 & 200002 & 20.19 & 20.68 & 24.73 & 22.33 & 19.89 \\
\hline 60 & ACO85J004204.90-094108.6 & 19.38 & 19.66 & 23.40 & 21.26 & 18.98 \\
\hline
\end{tabular}


Table A.4. continued.

\begin{tabular}{|c|c|c|c|c|c|c|}
\hline Num & Name & $\overline{\overline{R_{\mathrm{C}}}}$ & $\overline{\overline{r^{\prime}}}$ & $\overline{u^{*}}$ & $\overline{\overline{g^{\prime}}}$ & $\overline{\overline{i^{\prime}}}$ \\
\hline 61 & ACO85J004205.15-093715.5 & 20.20 & 20.59 & 22.91 & 21.68 & 20.03 \\
\hline 62 & ACO85J004205.67-094627.1 & 19.31 & 19.63 & 22.13 & 20.96 & 18.95 \\
\hline 63 & ACO85J004205.79-093026.0 & 19.74 & 20.19 & 24.13 & 21.84 & 19.37 \\
\hline 64 & ACO85J004205.86-094310.1 & 17.49 & 17.92 & 21.40 & 19.16 & 16.69 \\
\hline 65 & ACO85J004206.02-093606.4 & 16.20 & 16.38 & 18.62 & 17.10 & 15.99 \\
\hline 66 & ACO85J004207.26-093626.0 & 18.15 & 18.36 & 19.13 & 18.51 & 18.20 \\
\hline 67 & ACO85J004207.71-093059.3 & 18.31 & 18.84 & 22.92 & 20.52 & 18.09 \\
\hline 68 & ACO85J004208.27-092942.7 & 19.57 & 20.04 & 23.84 & 21.65 & 19.26 \\
\hline 69 & ACO85J004208.36-093104.6 & 17.87 & 18.20 & 19.81 & 18.68 & 17.89 \\
\hline 70 & ACO85J004208.67-093506.5 & 19.77 & 20.26 & 23.24 & 21.74 & 19.36 \\
\hline 71 & ACO85J004209.19-094056.6 & 20.32 & 20.77 & 23.04 & 21.93 & 19.91 \\
\hline 72 & ACO85J004209.81-092852.2 & 17.65 & 17.94 & 18.97 & 18.17 & 17.73 \\
\hline 73 & ACO85J004210.63-093129.7 & 20.35 & 20.80 & 24.54 & 22.33 & 19.66 \\
\hline 74 & ACO85J004214.92-092735.4 & 19.74 & 20.12 & 23.84 & 21.67 & 19.37 \\
\hline 75 & ACO85J004215.64-094209.2 & 20.32 & 20.82 & 24.26 & 22.27 & 19.77 \\
\hline 76 & ACO85J004216.93-093325.6 & 19.91 & 20.05 & 23.30 & 21.13 & 19.06 \\
\hline 77 & ACO85J004217.94-093620.9 & 20.71 & 21.23 & 24.82 & 22.51 & 19.48 \\
\hline 78 & ACO85J004218.47-093912.1 & 17.50 & - & - & - & - \\
\hline 79 & ACO85J004218.55-093910.2 & 19.55 & - & - & - & - \\
\hline 80 & ACO85J004219.89-092527.5 & 17.20 & 17.37 & 19.03 & 17.87 & 17.08 \\
\hline 81 & ACO85J004220.58-093526.4 & 19.88 & 20.11 & 21.44 & 20.77 & 19.73 \\
\hline 82 & ACO85J004220.87-094517.5 & 20.43 & 20.65 & 24.51 & 22.20 & 19.59 \\
\hline 83 & ACO85J004224.68-092716.2 & 20.00 & 20.29 & 21.34 & 20.84 & 20.09 \\
\hline 84 & ACO85J004224.74-093741.3 & 20.48 & 20.68 & 24.35 & 22.29 & 19.91 \\
\hline 85 & ACO85J004225.48-093538.6 & 20.00 & 20.32 & 24.14 & 21.81 & 19.35 \\
\hline 86 & ACO85J004225.54-093708.9 & 17.47 & 17.54 & 19.91 & 18.29 & 17.15 \\
\hline 87 & ACO85J004226.35-093629.5 & 19.67 & 19.82 & 23.16 & 21.35 & 19.08 \\
\hline 88 & ACO85J004227.26-093116.5 & 19.43 & 20.12 & 23.63 & 21.37 & 18.91 \\
\hline 89 & ACO85J004227.58-095059.3 & 20.19 & 20.38 & 23.01 & 21.52 & 18.92 \\
\hline 90 & ACO85J004228.38-094938.3 & 17.66 & 17.79 & 19.65 & 18.38 & 17.47 \\
\hline 91 & ACO85J004228.83-094523.9 & 20.08 & 20.25 & 24.10 & 21.83 & 19.51 \\
\hline 92 & ACO85J004232.84-092144.2 & 18.37 & - & - & - & - \\
\hline 93 & ACO85J004233.31-094448.0 & 19.58 & 19.68 & 22.77 & 21.18 & 18.99 \\
\hline 94 & ACO85J004236.76-094403.8 & 18.69 & 18.84 & 20.09 & 19.18 & 18.55 \\
\hline 95 & ACO85J004237.07-094520.5 & 17.83 & 18.02 & 19.71 & 18.61 & 17.65 \\
\hline 96 & ACO85J004238.03-093229.9 & 20.36 & 20.75 & 24.49 & 22.39 & 19.69 \\
\hline 97 & ACO85J004242.24-092108.7 & 21.39 & - & - & - & - \\
\hline 98 & ACO85J004242.54-094726.4 & 17.42 & 17.80 & 21.30 & 19.02 & 16.26 \\
\hline 99 & ACO85J004243.90-094420.8 & 16.12 & 16.34 & 18.54 & 17.05 & 15.96 \\
\hline 100 & ACO85J004245.68-092327.8 & 21.07 & - & - & - & $-\mathrm{R}$ \\
\hline 101 & ACO85J004247.85-092522.6 & 21.13 & 22.01 & 25.46 & 23.21 & 19.93 \\
\hline
\end{tabular}

Table A.5. Broad band magnitudes of the 6 galaxies with $\mathrm{H} \alpha$ emission in their SDSS spectra but not detected in our narrow band filter due to its wavelength cut.

\begin{tabular}{rlrrrr}
\hline \hline Number & Name & $z_{\text {SDSS }}$ & $r_{\text {SDSS }}$ & $\begin{array}{r}E W(\mathrm{H} \alpha) \\
(\AA)\end{array}$ & $\begin{array}{r}\Delta E W(\mathrm{H} \alpha) \\
(\AA)\end{array}$ \\
\hline 1 & ACO85J004119.01-092323.50 & 0.0498 & 17.37 & 23.9 & 0.4 \\
2 & ACO85J004127.86-092329.54 & 0.0494 & 17.43 & 4.5 & 0.3 \\
3 & ACO85J004218.46-093912.10 & 0.0515 & 17.69 & 64.4 & 2.4 \\
4 & ACO85J004219.90-092527.55 & 0.0511 & 17.42 & 23.4 & 0.3 \\
5 & ACO85J004228.37-094938.28 & 0.0500 & 17.76 & 20.0 & 0.7 \\
6 & ACO85J004243.90-094420.83 & 0.0507 & 16.28 & 7.7 & 0.2 \\
\hline
\end{tabular}


G. Boué et al.: Filament of Abell 85, Online Material p 7

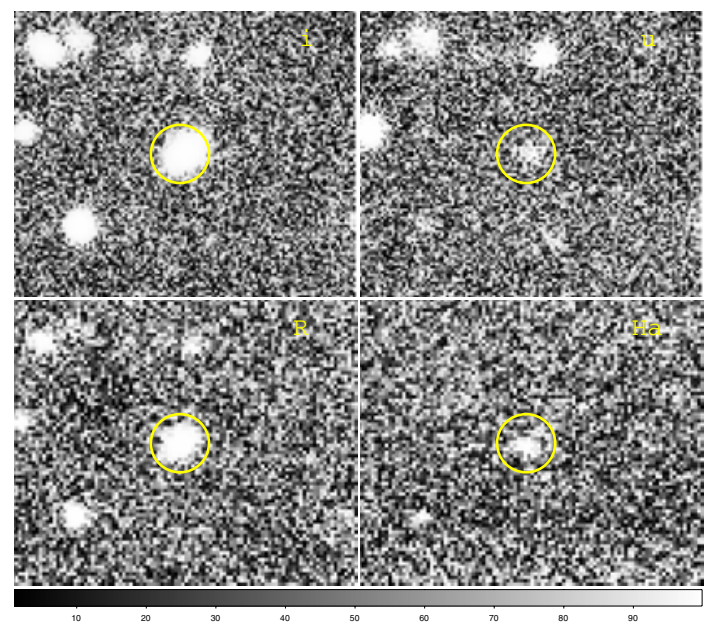

Fig. A.1. Images of galaxy ACO85J004037.99-095038.8, in the $i^{\prime}$ (top left), $u^{*}$ (top right), $R_{\mathrm{C}}$ (bottom left) and "pure" $\mathrm{H} \alpha$ (bottom right) bands. The circles (with radius of $2^{\prime \prime}$ ) indicate the position of the galaxy detected in the $R_{\mathrm{C}}$ band (they do not indicate the region in which the $\mathrm{H} \alpha$ fluxes were measured). Each image is $23.3^{\prime \prime} \times 19.5^{\prime \prime}$.

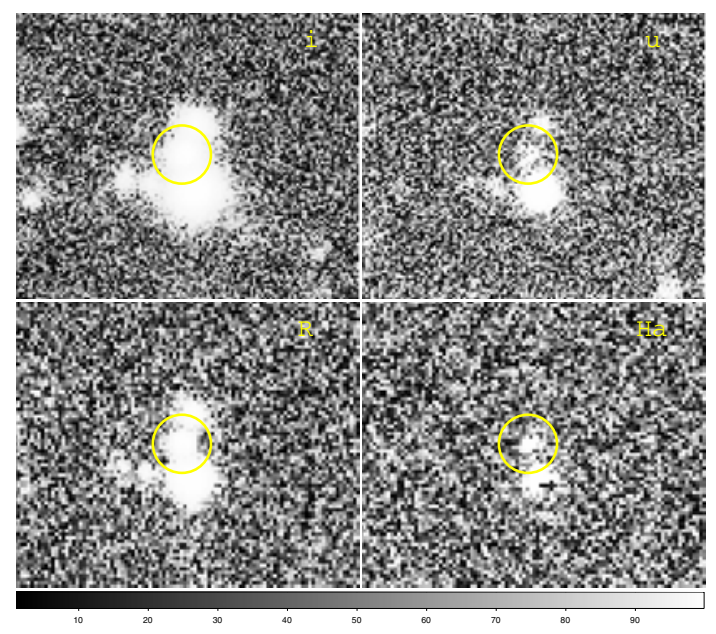

Fig. A.2. Same as Fig. 1 for galaxy ACO85J004039.27-092933.8.

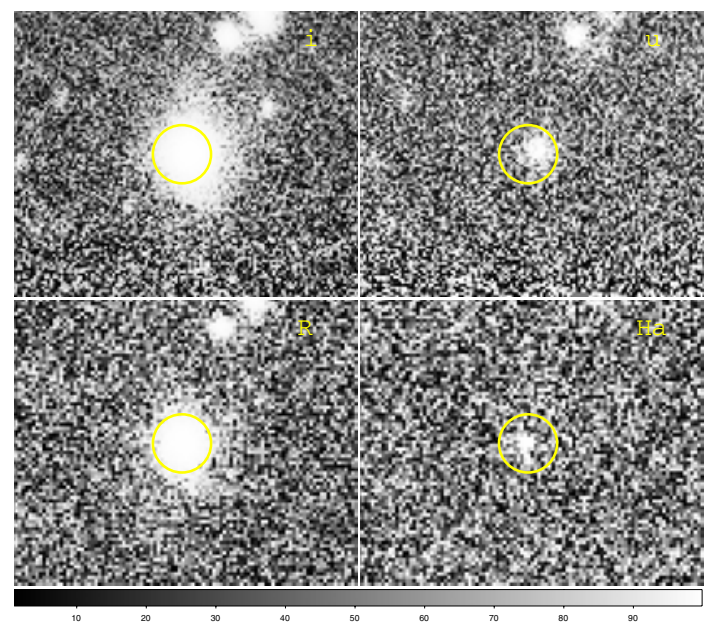

Fig. A.3. Same as Fig. 1 for galaxy ACO85J004039.90-093812.5.

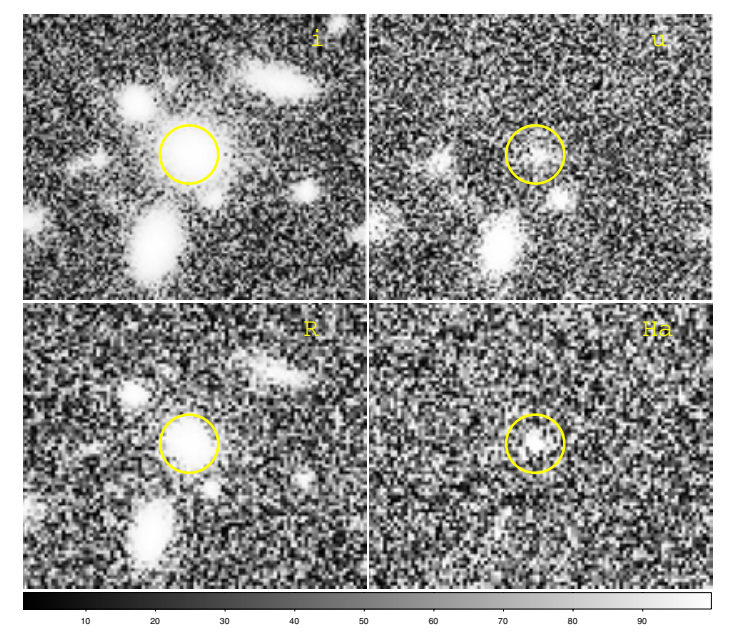

Fig. A.4. Same as Fig. 1 for galaxy ACO85J004041.11-093054.7.

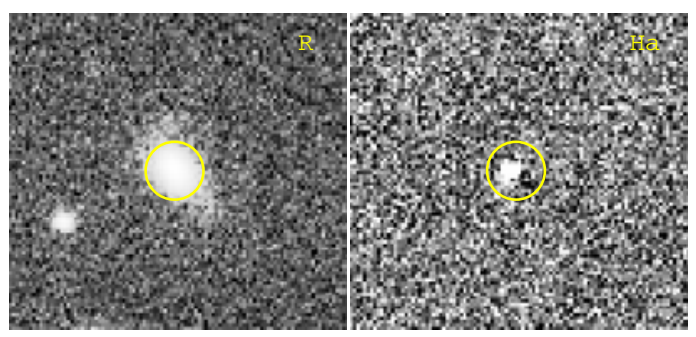

Fig. A.5. Same as Fig. 1 for galaxy ACO85J004043.91-092101.7.

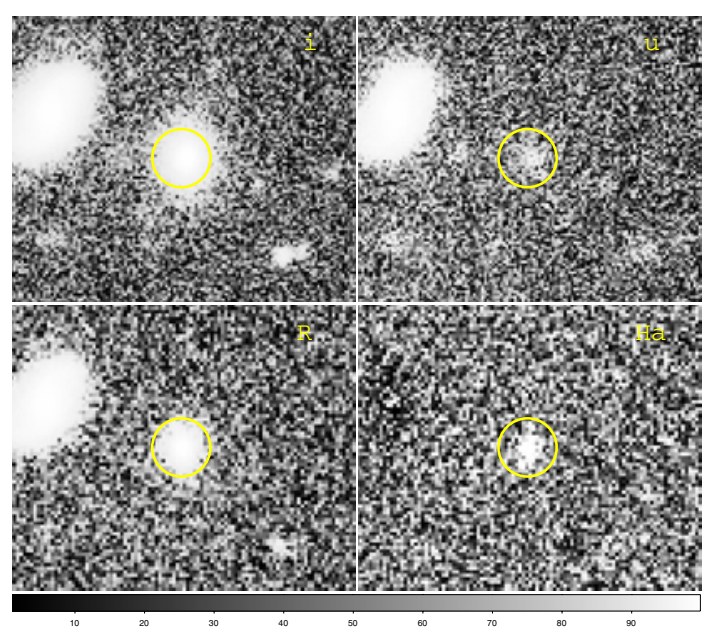

Fig. A.6. Same as Fig. 1 for galaxy ACO85J004044.33-093334.4. 
G. Boué et al.: Filament of Abell 85, Online Material $p 8$

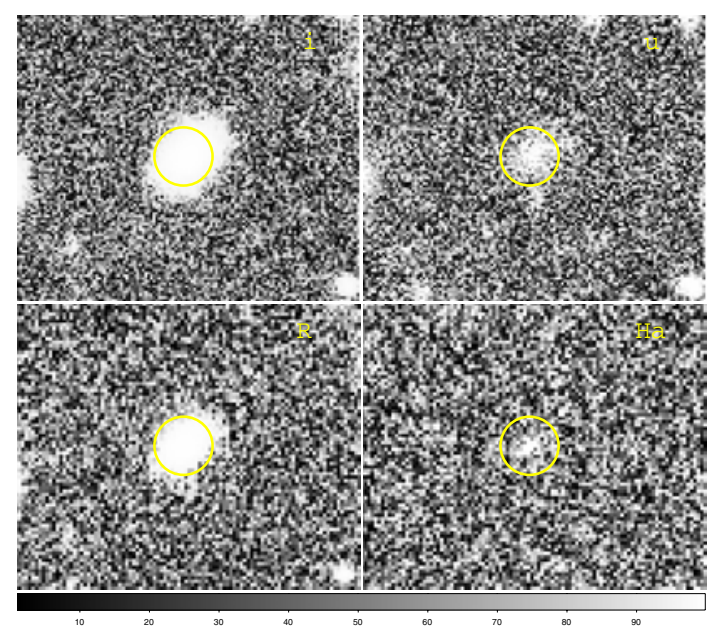

Fig. A.7. Same as Fig. 1 for galaxy ACO85J004045.38-093140.9.

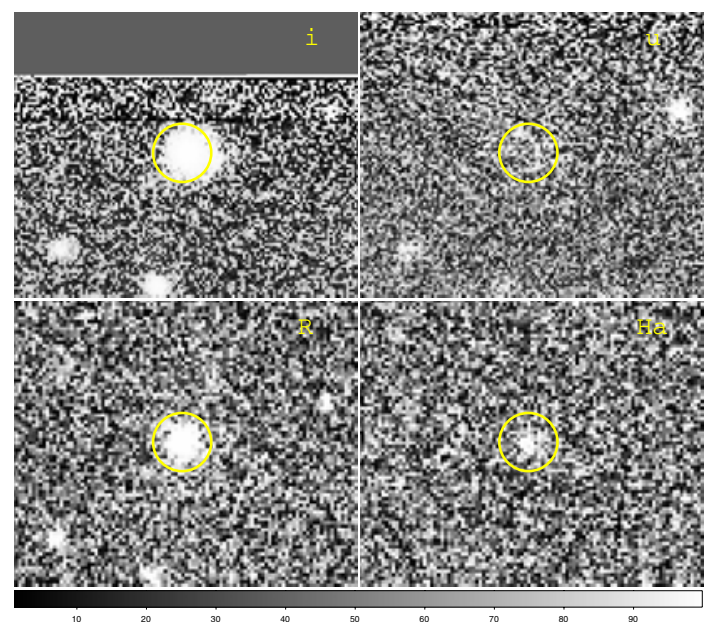

Fig. A.8. Same as Fig. 1 for galaxy ACO85J004045.80-093929.1.

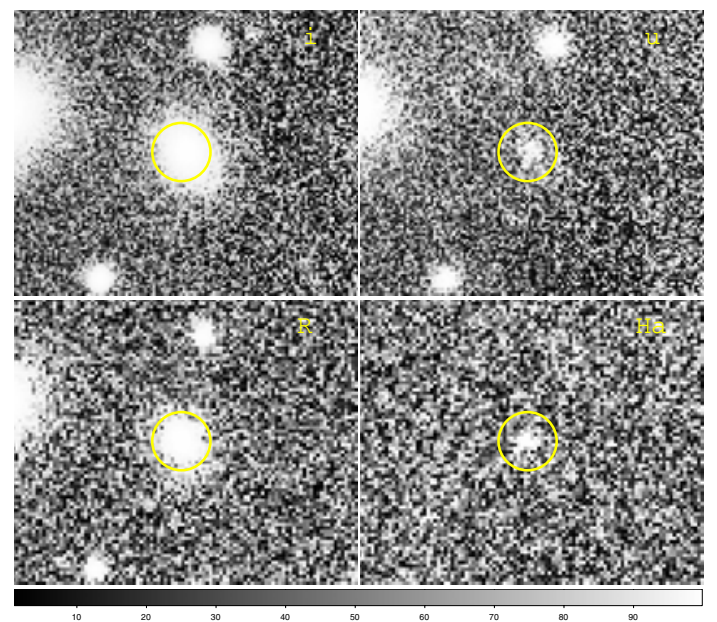

Fig. A.9. Same as Fig. 1 for galaxy ACO85J004050.05-094402.5.

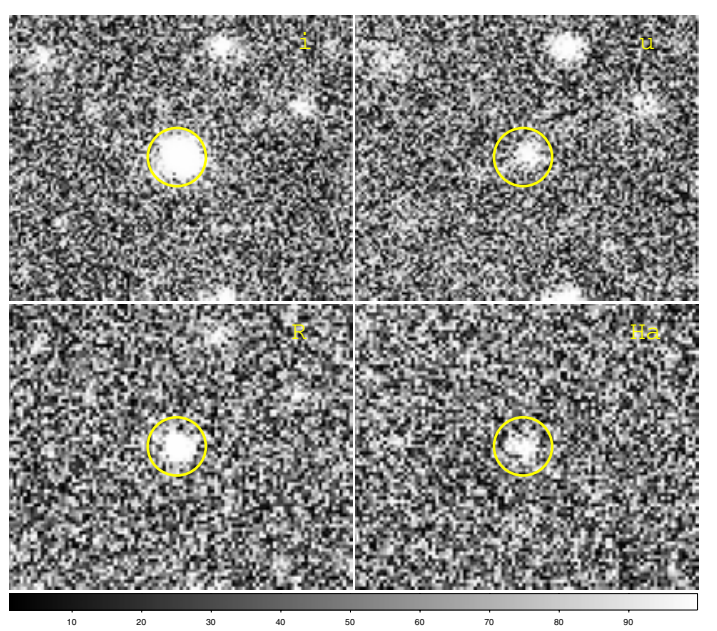

Fig. A.10. Same as Fig. 1 for galaxy ACO85J004053.86-094845.6.

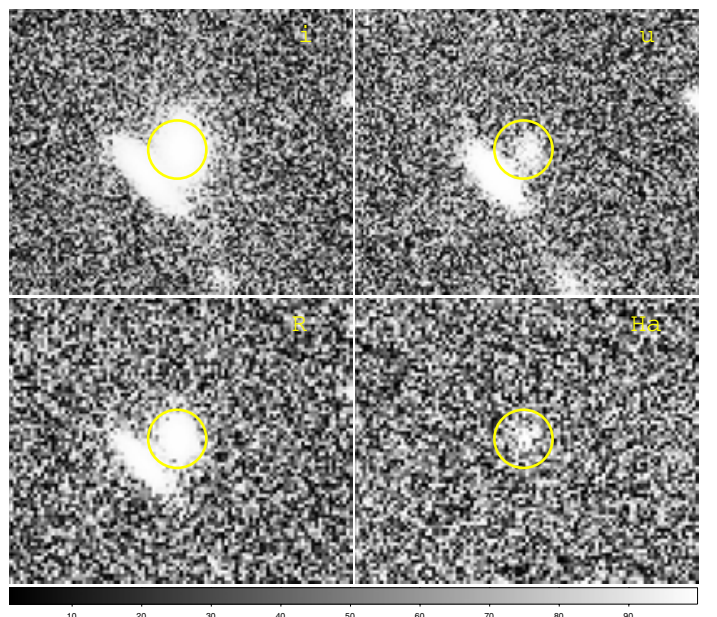

Fig. A.11. Same as Fig. 1 for galaxy ACO85J004055.06-094954.9.

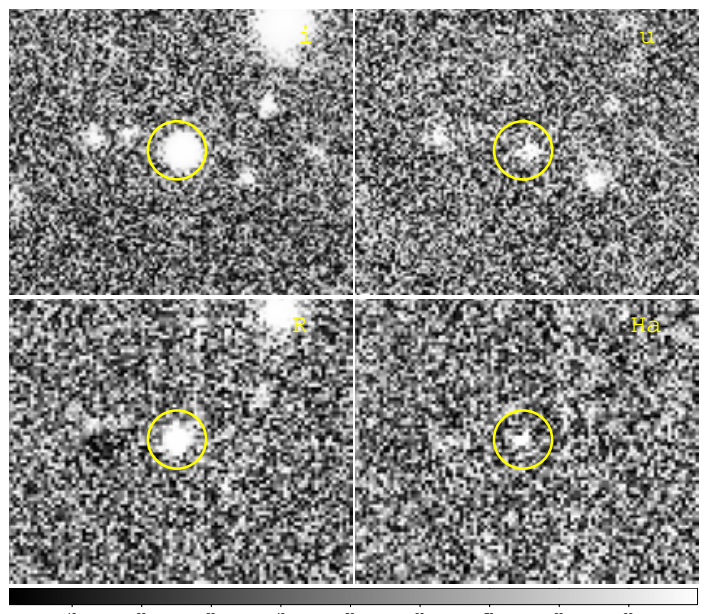

Fig. A.12. Same as Fig. 1 for galaxy ACO85J004103.65-093342.1. 
G. Boué et al.: Filament of Abell 85, Online Material p 9

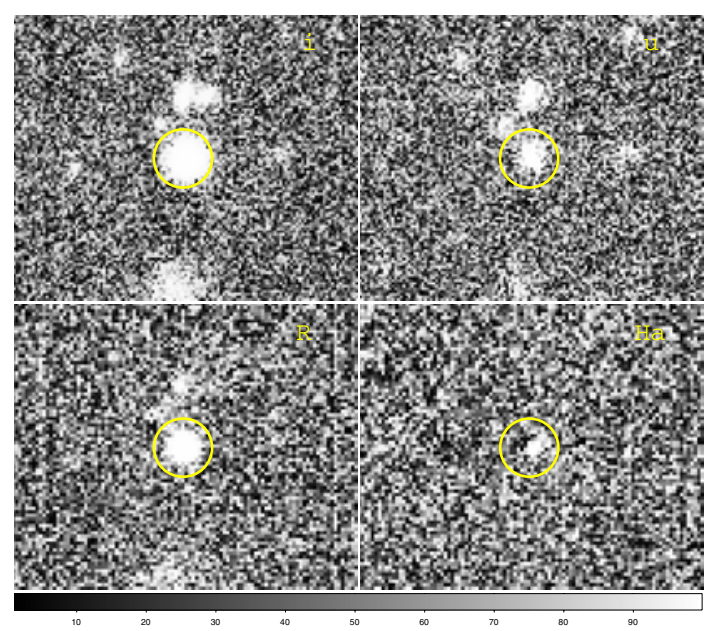

Fig. A.13. Same as Fig. 1 for galaxy ACO85J004104.27-093627.8.

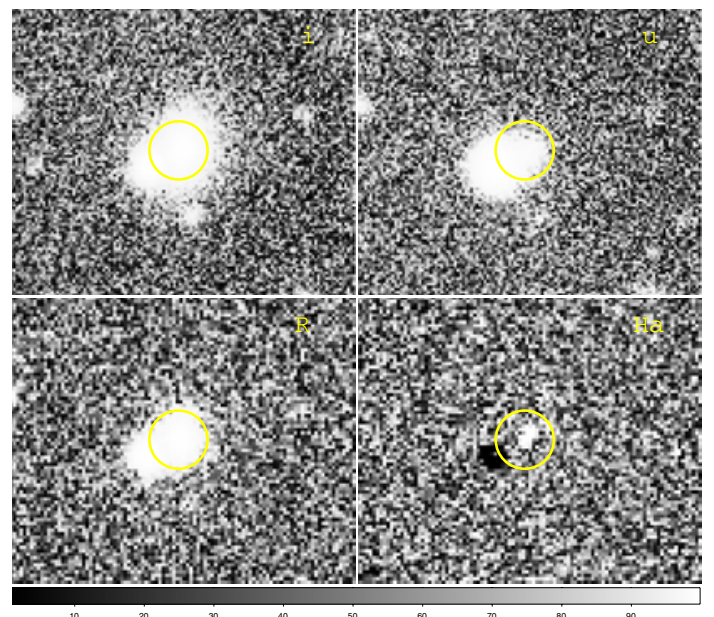

Fig. A.14. Same as Fig. 1 for galaxy ACO85J004104.67-092608.6.

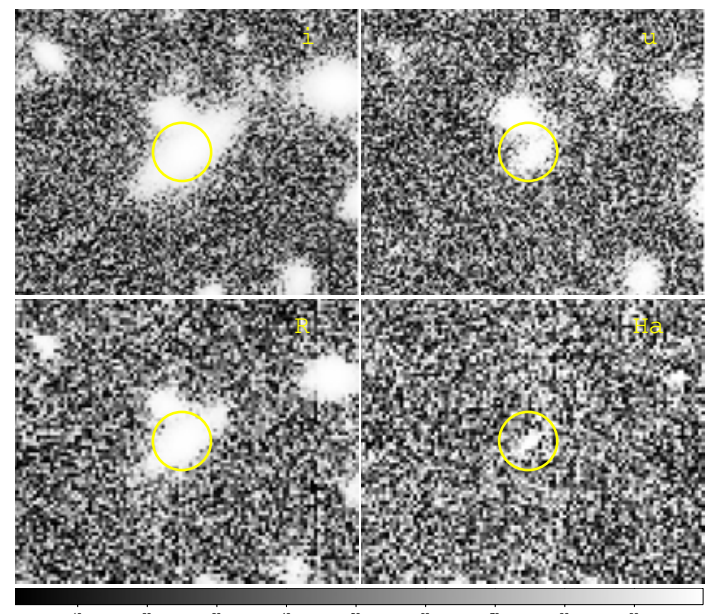

Fig. A.15. Same as Fig. 1 for galaxy ACO85J004106.35-094321.1.

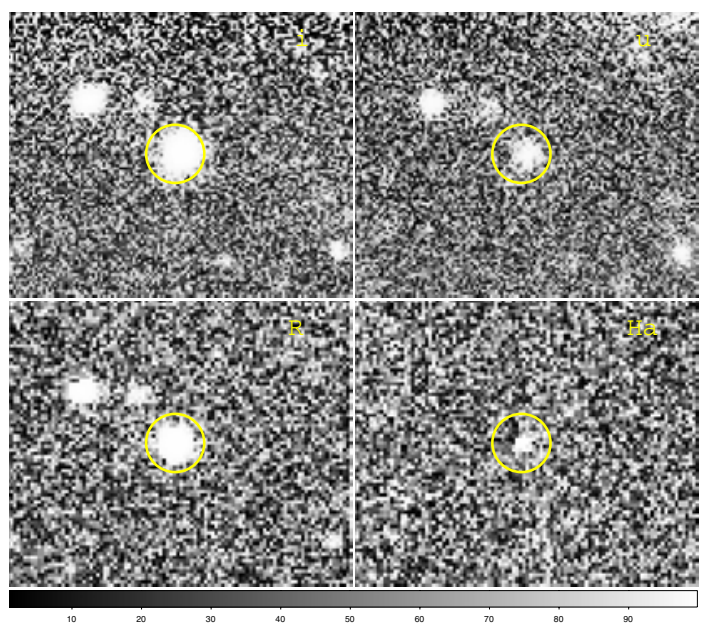

Fig. A.16. Same as Fig. 1 for galaxy ACO85J004108.66-093932.2.
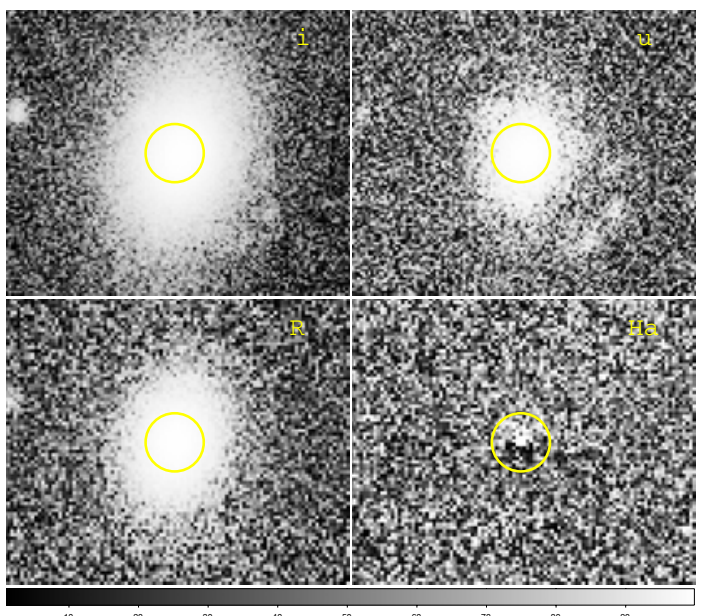

Fig. A.17. Same as Fig. 1 for galaxy ACO85J004109.80-093503.0.

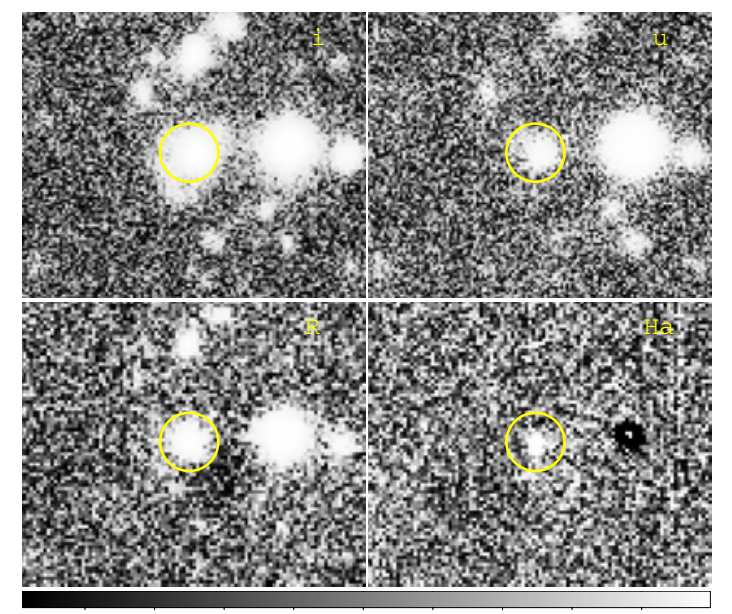

Fig. A.18. Same as Fig. 1 for galaxy ACO85J004110.45-093247.7. 
G. Boué et al.: Filament of Abell 85, Online Material $p 10$

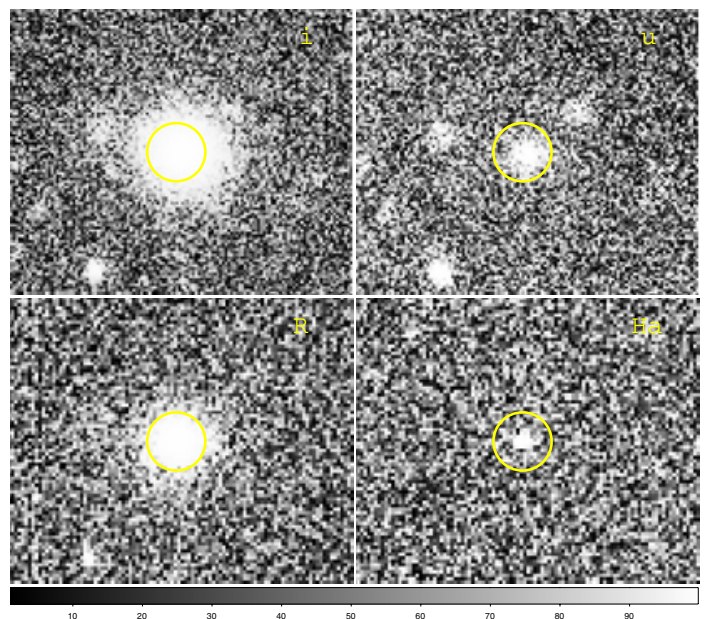

Fig. A.19. Same as Fig. 1 for galaxy ACO85J004113.96-094015.3.
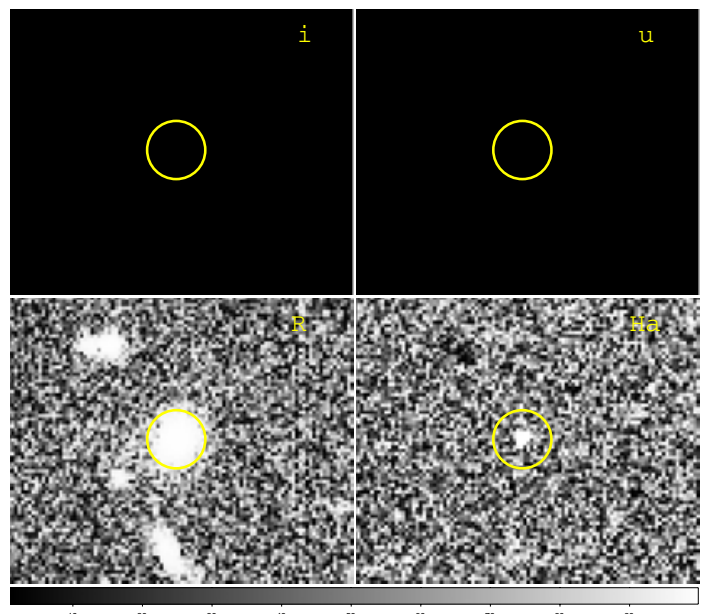

Fig. A.20. Same as Fig. 1 for galaxy ACO85J004115.20-093856.8.

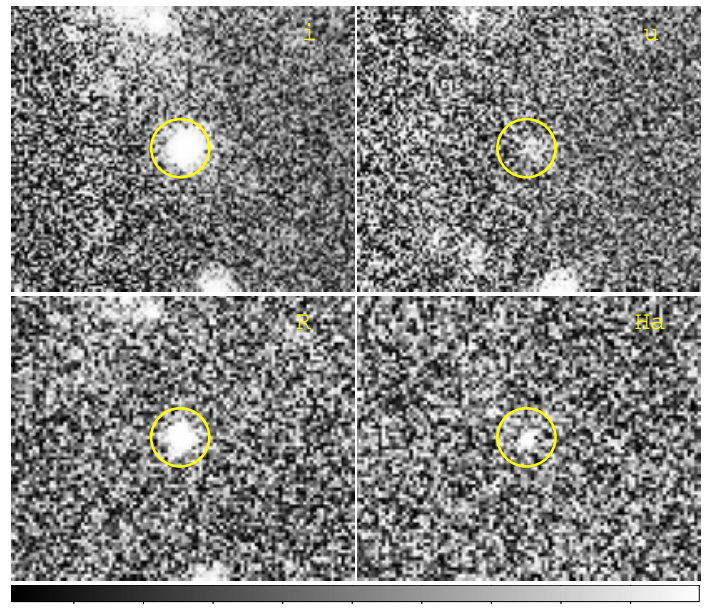

Fig. A.21. Same as Fig. 1 for galaxy ACO85J004115.27-093053.9.

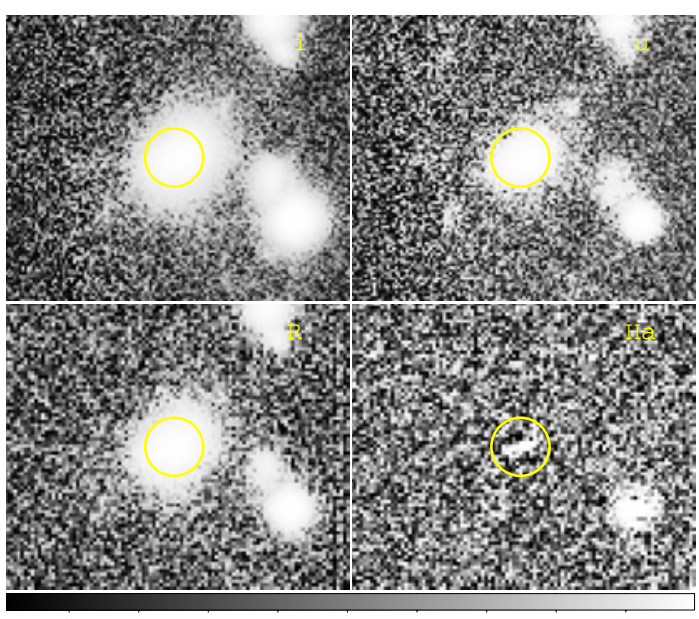

Fig. A.22. Same as Fig. 1 for galaxy ACO85J004115.38-094134.6.

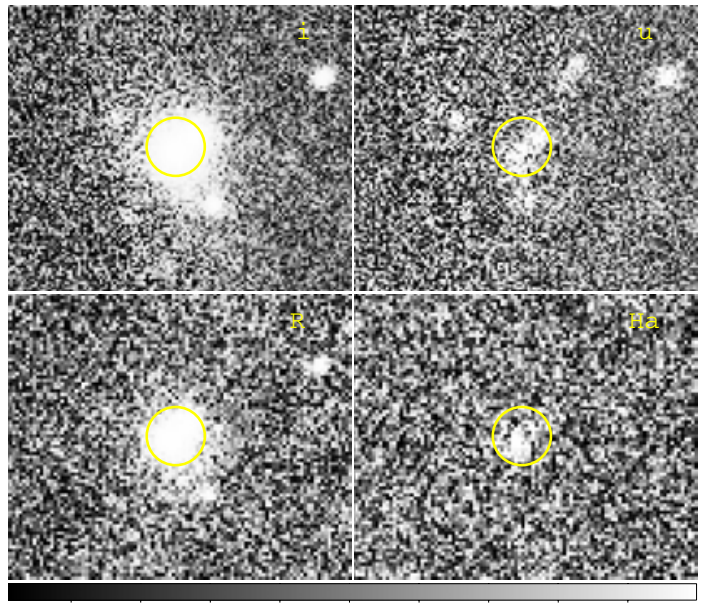

Fig. A.23. Same as Fig. 1 for galaxy ACO85J004115.55-093041.3.

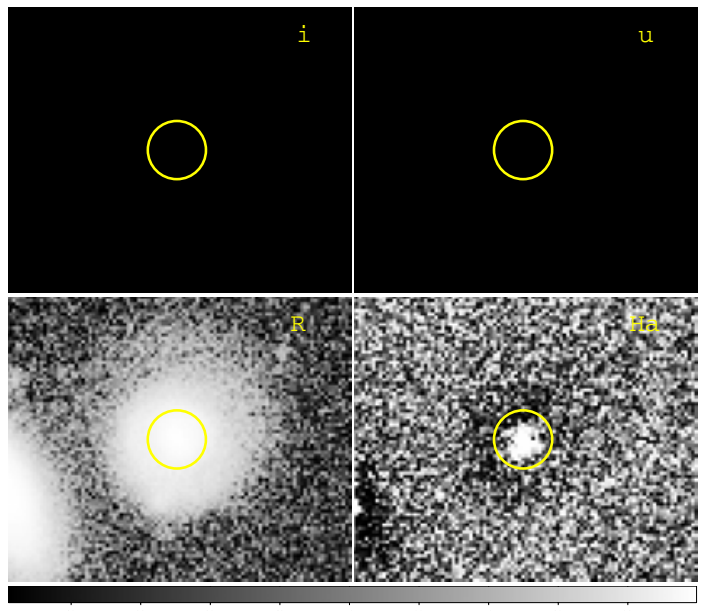

Fig. A.24. Same as Fig. 1 for galaxy ACO85J004119.01-092323.5. 
G. Boué et al.: Filament of Abell 85, Online Material p 11

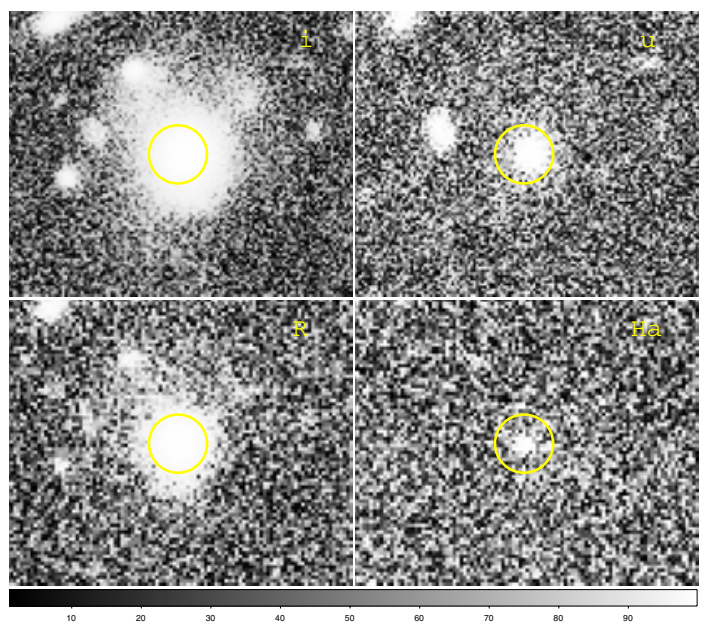

Fig. A.25. Same as Fig. 1 for galaxy ACO85J004119.11-093312.0.
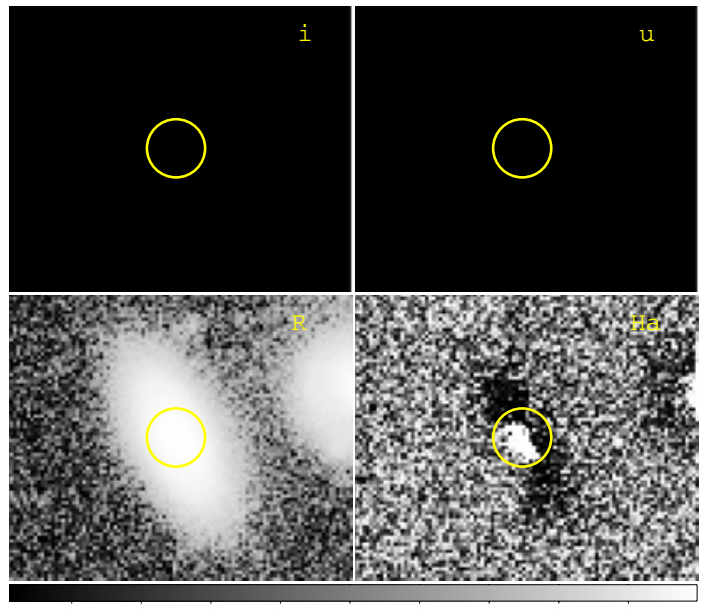

Fig. A.26. Same as Fig. 1 for galaxy ACO85J004119.83-092327.0.

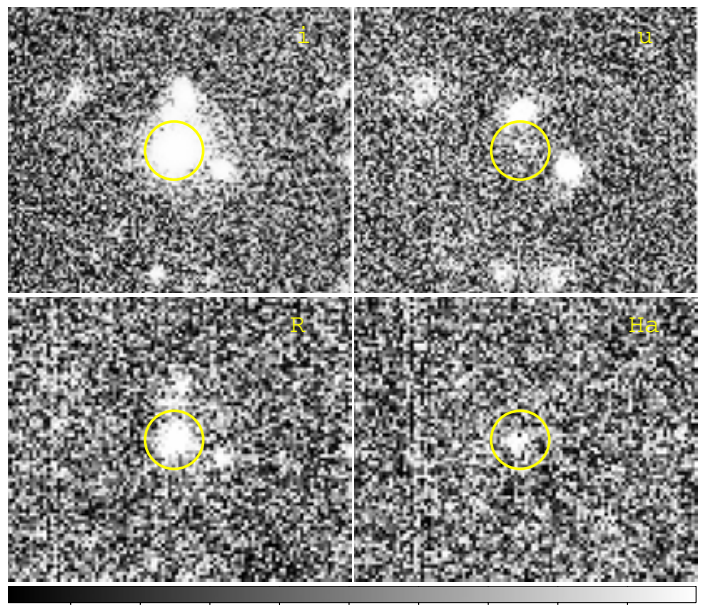

Fig. A.27. Same as Fig. 1 for galaxy ACO85J004122.02-094156.0.

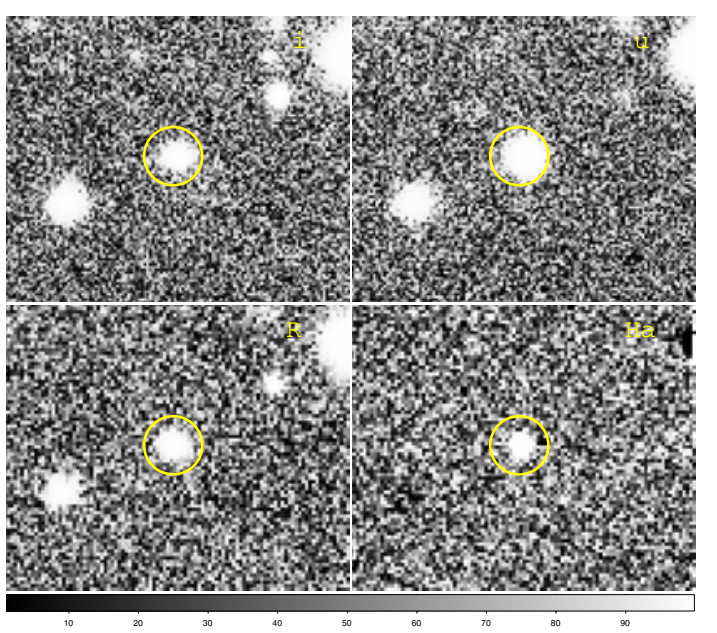

Fig. A.28. Same as Fig. 1 for galaxy ACO85J004122.17-092639.5.

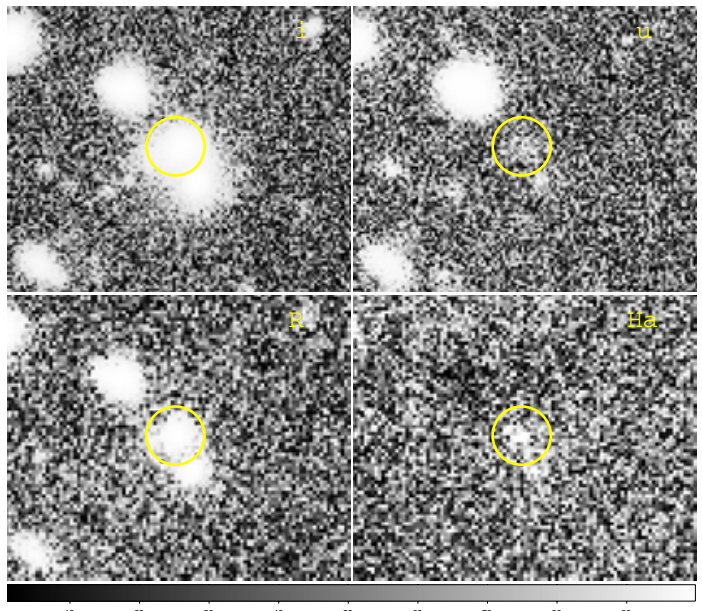

Fig. A.29. Same as Fig. 1 for galaxy ACO85J004123.20-093208.6.

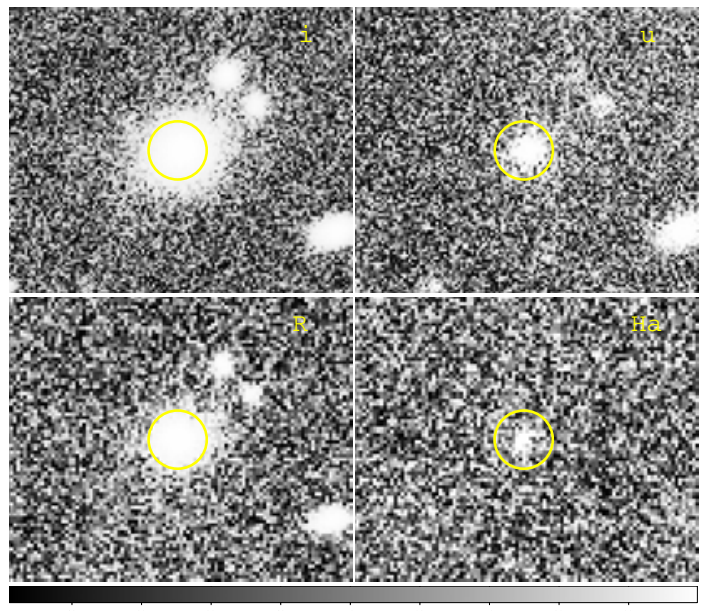

Fig. A.30. Same as Fig. 1 for galaxy ACO85J004124.32-092600.2. 
G. Boué et al.: Filament of Abell 85, Online Material p 12

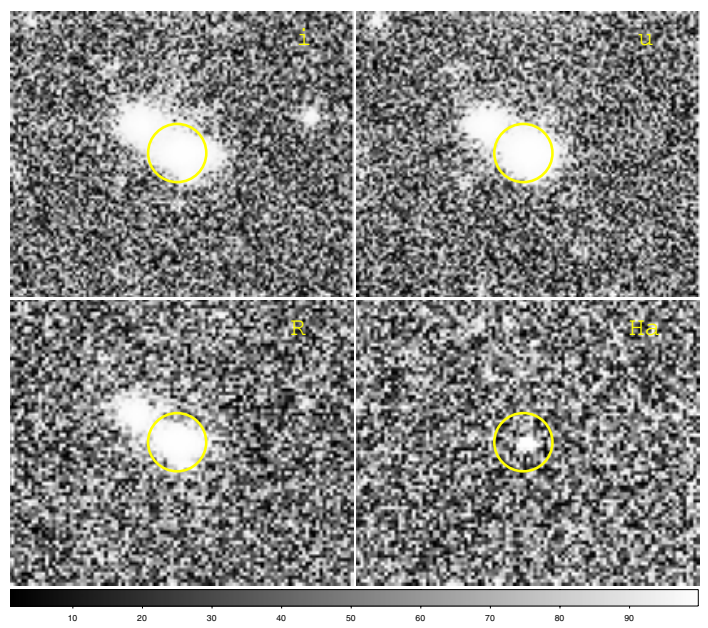

Fig. A.31. Same as Fig. 1 for galaxy ACO85J004124.48-093405.3.

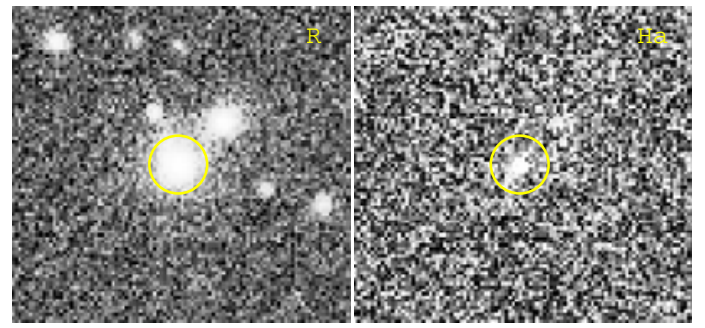

Fig. A.32. Same as Fig. 1 for galaxy ACO85J004126.27-092101.5.

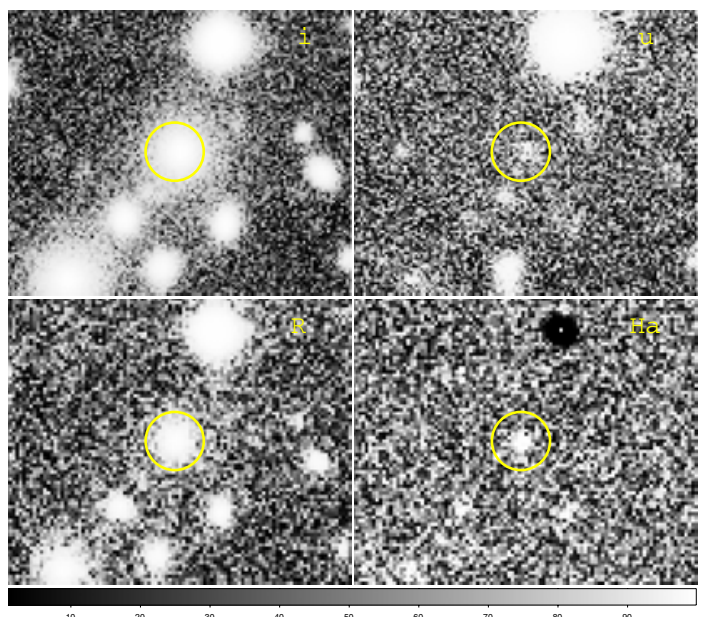

Fig. A.33. Same as Fig. 1 for galaxy ACO85J004127.13-092857.6.

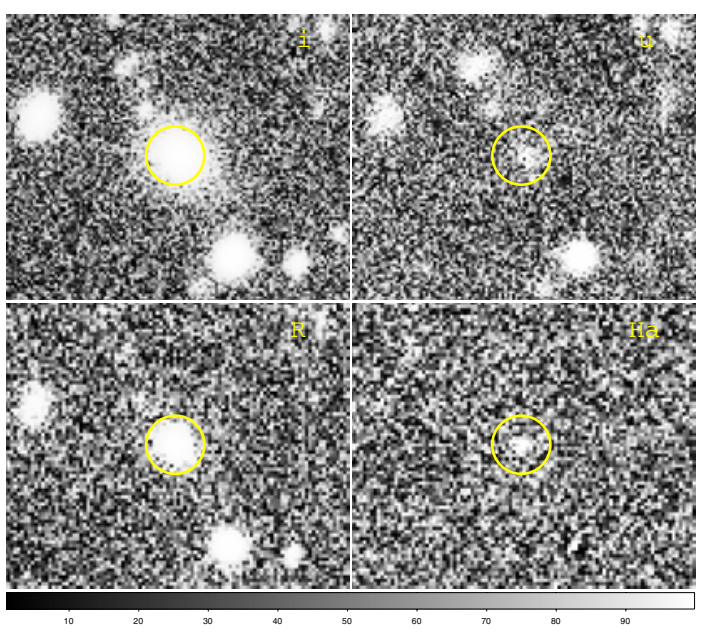

Fig. A.34. Same as Fig. 1 for galaxy ACO85J004128.95-092837.1.

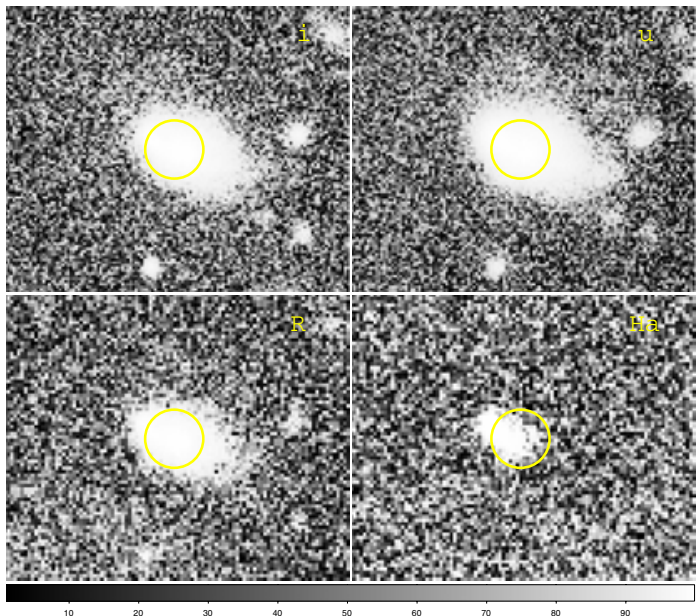

Fig. A.35. Same as Fig. 1 for galaxy ACO85J004129.77-093313.2.

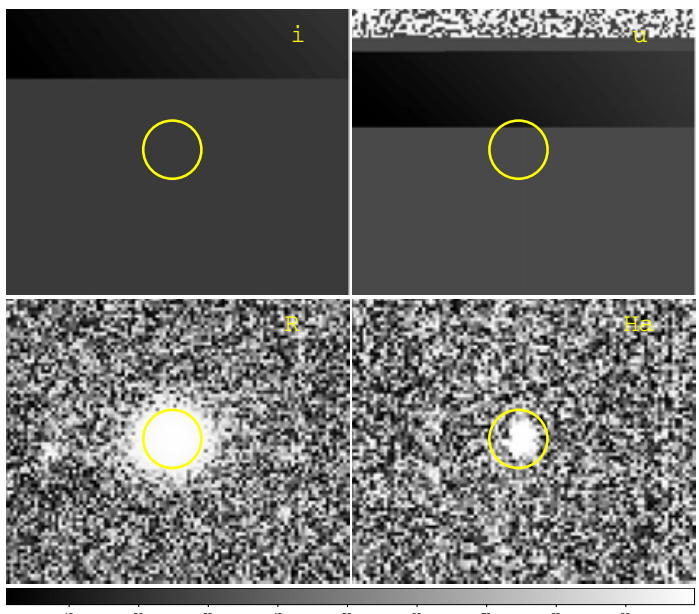

Fig. A.36. Same as Fig. 1 for galaxy ACO85J004131.77-093832.1. 
G. Boué et al.: Filament of Abell 85, Online Material p 13

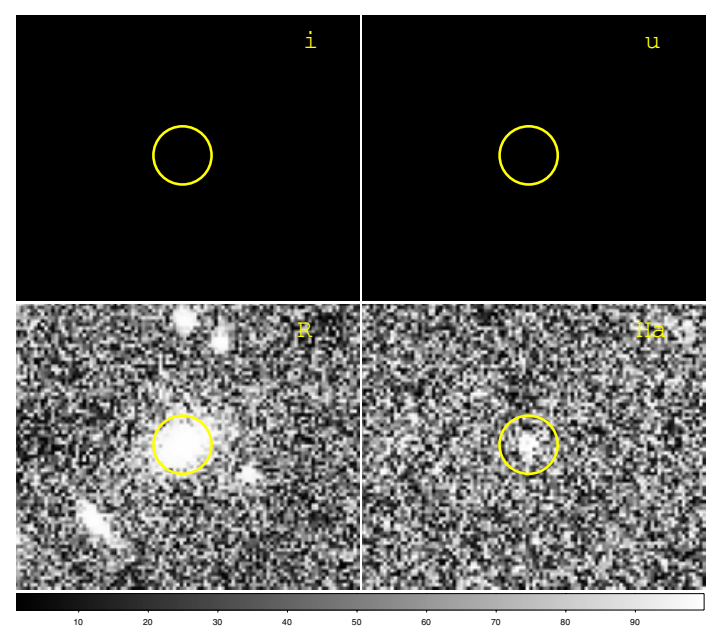

Fig. A.37. Same as Fig. 1 for galaxy ACO85J004131.80-092303.7.

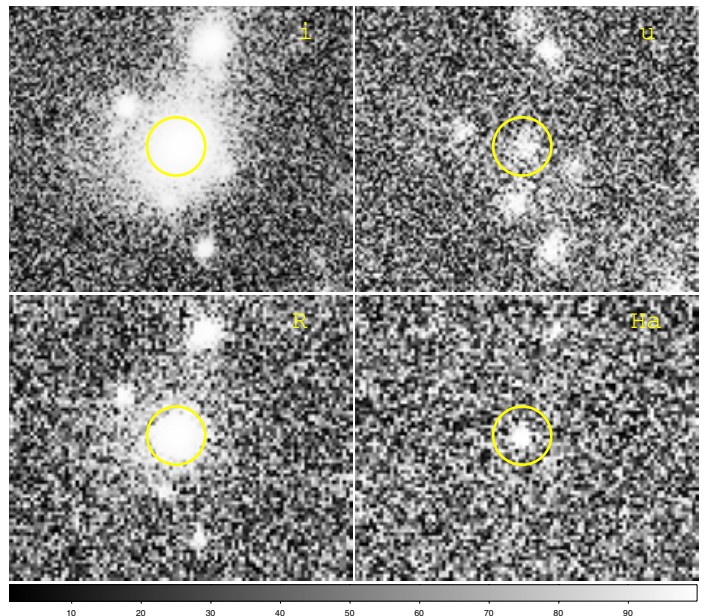

Fig. A.38. Same as Fig. 1 for galaxy ACO85J004132.70-092800.7.

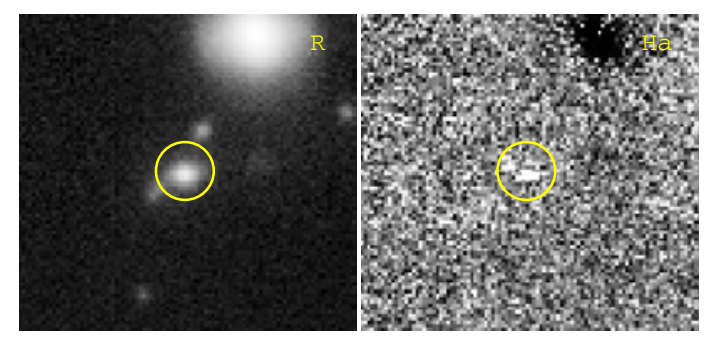

Fig. A.39. Same as Fig. 1 for galaxy ACO85J004136.55-091939.5.

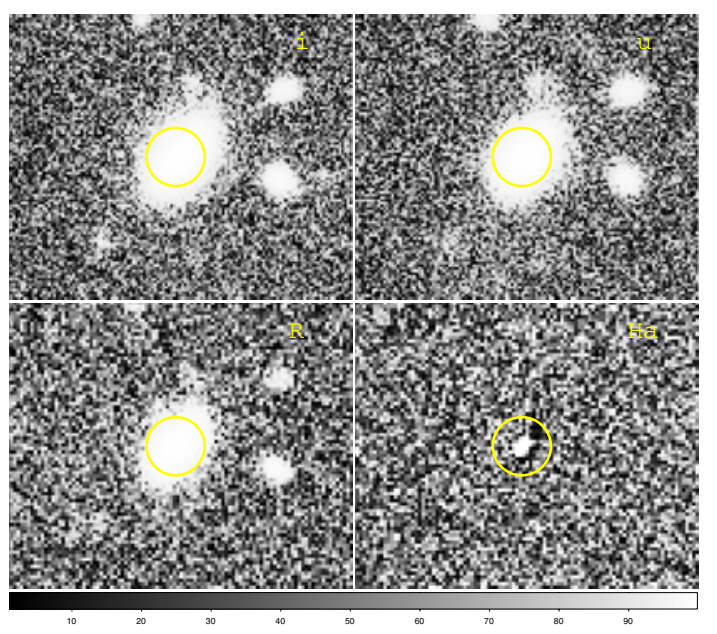

Fig. A.40. Same as Fig. 1 for galaxy ACO85J004138.01-092938.0.
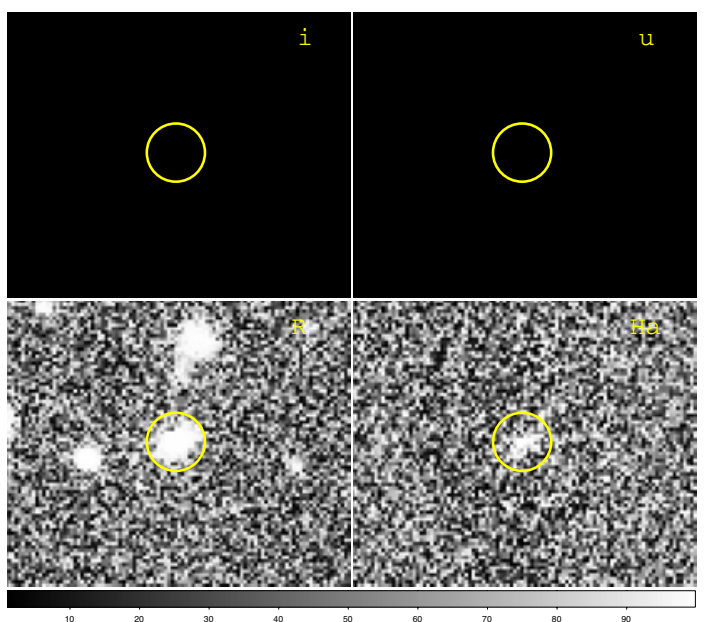

Fig. A.41. Same as Fig. 1 for galaxy ACO85J004139.37-092316.3.

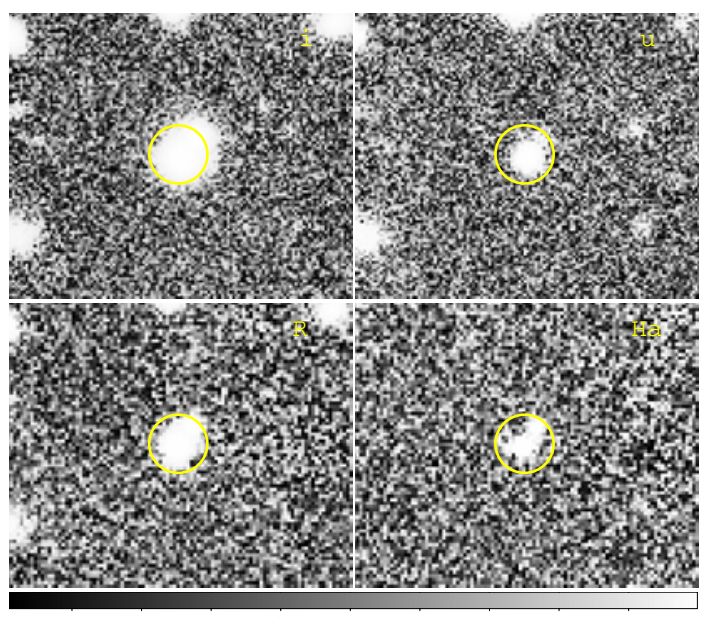

Fig. A.42. Same as Fig. 1 for galaxy ACO85J004140.92-093454.9. 
G. Boué et al.: Filament of Abell 85, Online Material p 14

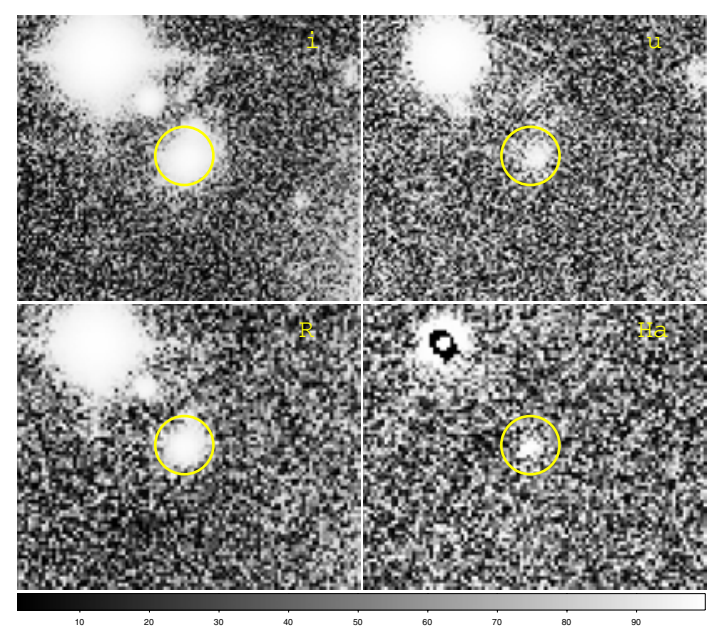

Fig. A.43. Same as Fig. 1 for galaxy ACO85J004141.04-092444.9.

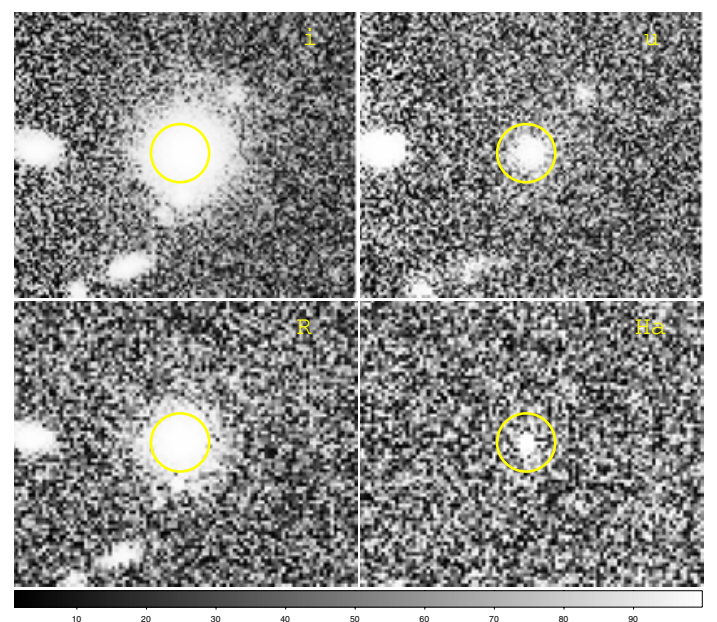

Fig. A.44. Same as Fig. 1 for galaxy ACO85J004141.67-093409.5.

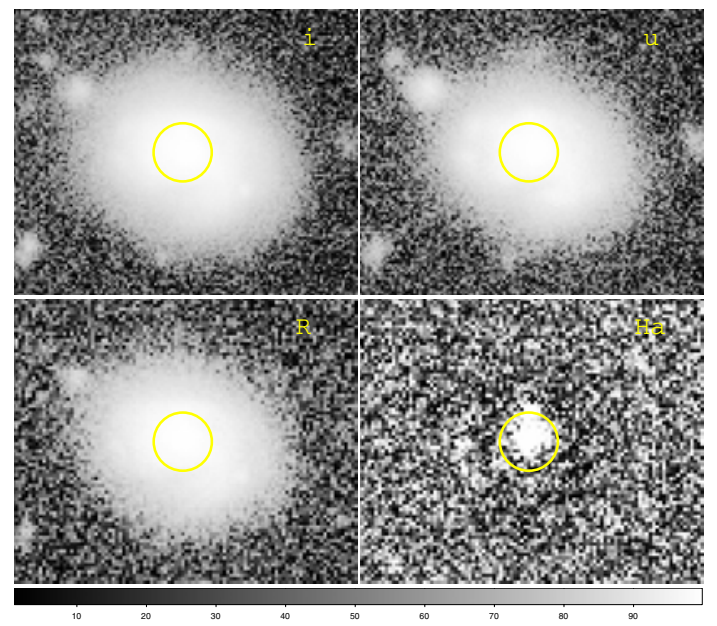

Fig. A.45. Same as Fig. 1 for galaxy ACO85J004145.45-094033.1.

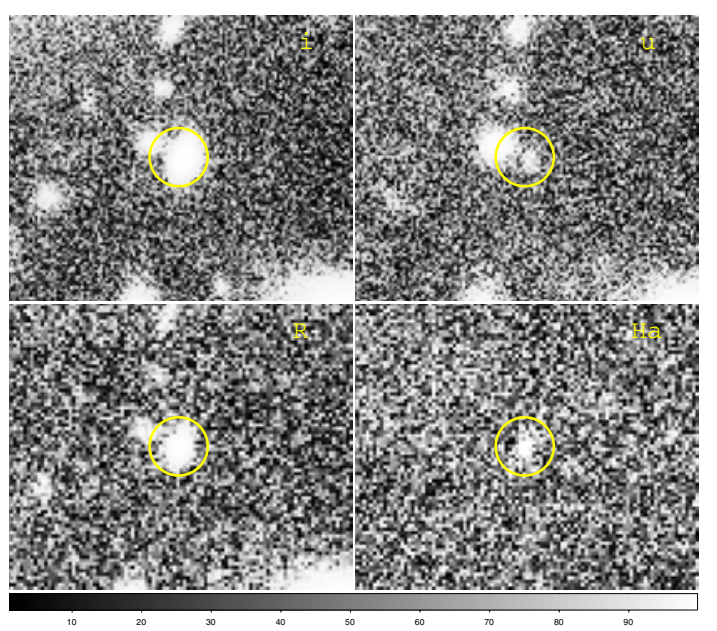

Fig. A.46. Same as Fig. 1 for galaxy ACO85J004148.91-092618.7.
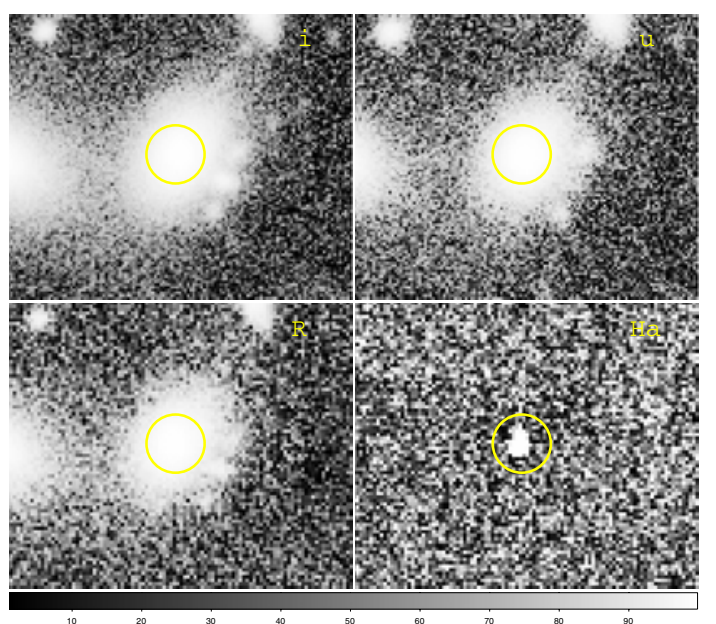

Fig. A.47. Same as Fig. 1 for galaxy ACO85J004149.38-092818.3.

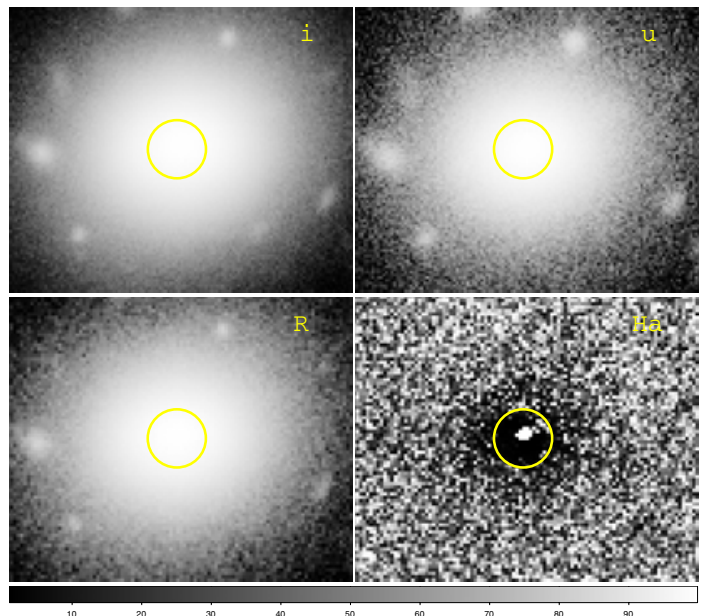

Fig. A.48. Same as Fig. 1 for galaxy ACO85J004150.17-092547.6. 
G. Boué et al.: Filament of Abell 85, Online Material p 15

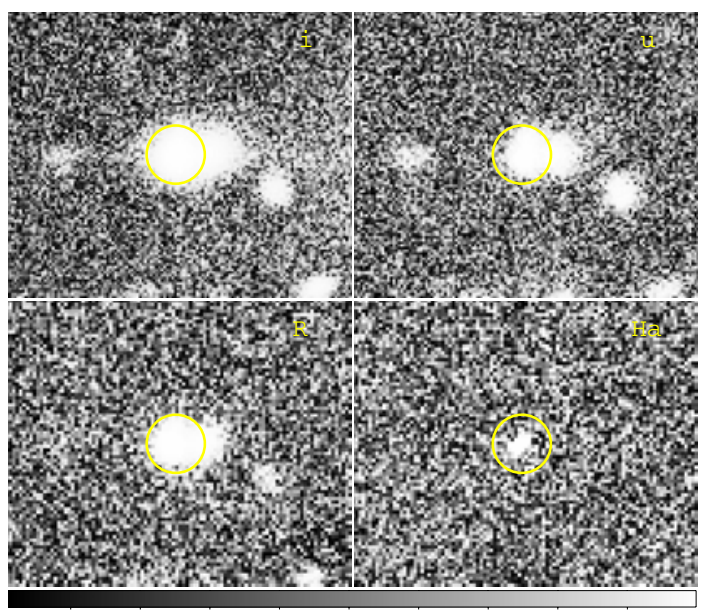

Fig. A.49. Same as Fig. 1 for galaxy ACO85J004150.75-092714.8.

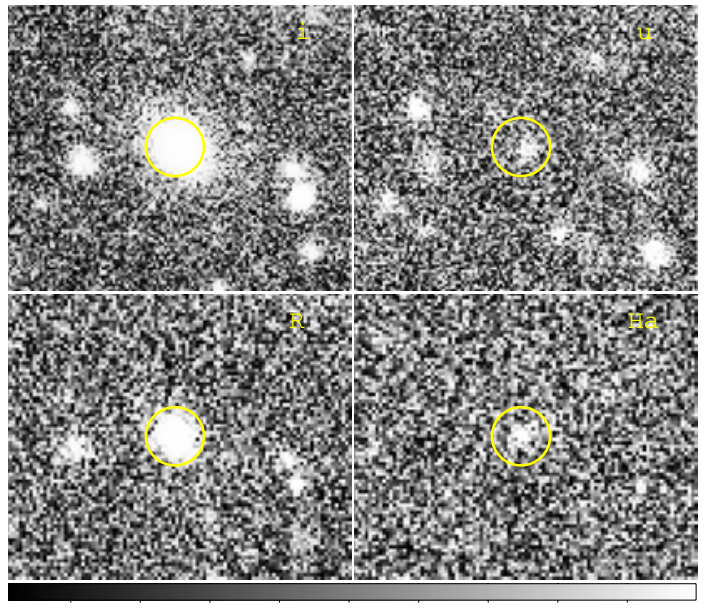

Fig. A.50. Same as Fig. 1 for galaxy ACO85J004150.88-092836.9.

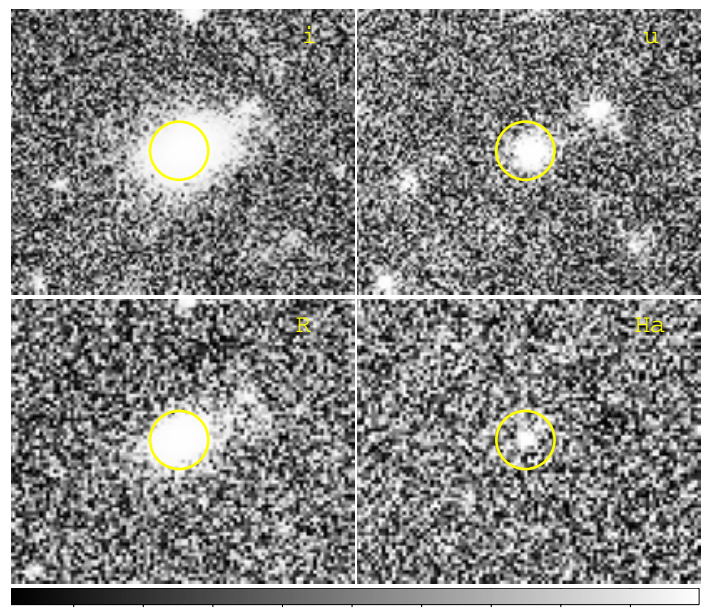

Fig. A.51. Same as Fig. 1 for galaxy ACO85J004150.94-092938.1.

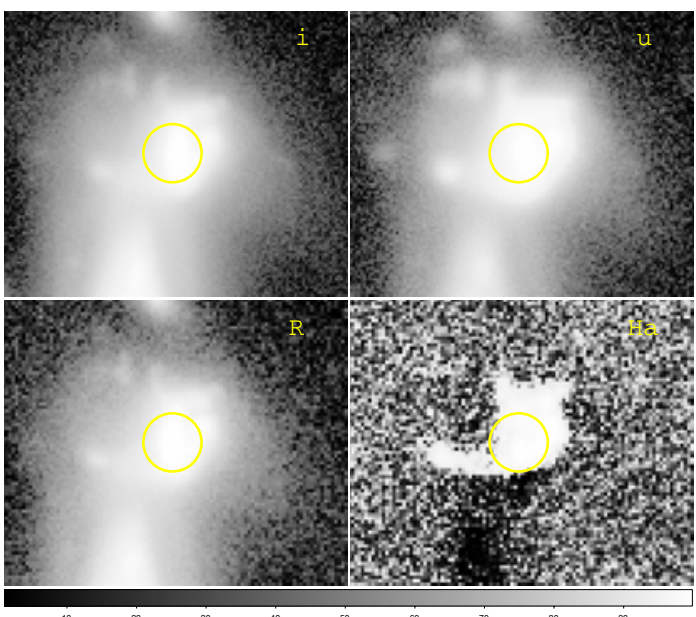

Fig. A.52. Same as Fig. 1 for galaxy ACO85J004153.27-092930.4.
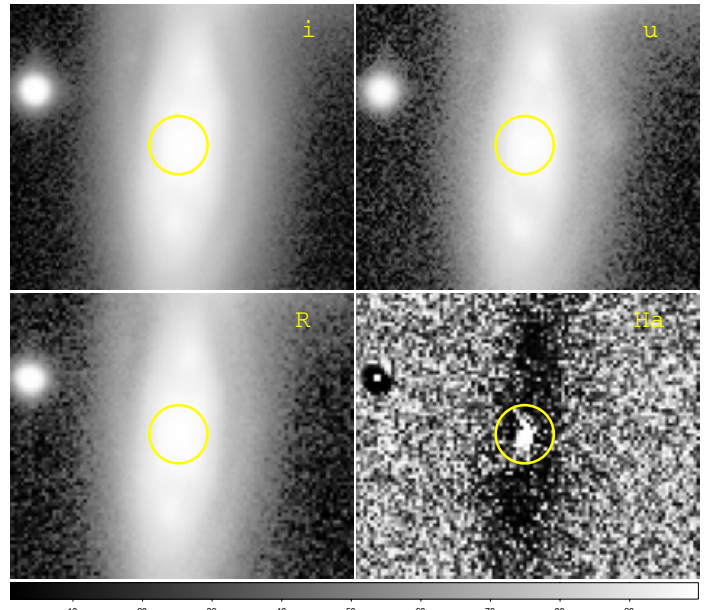

Fig. A.53. Same as Fig. 1 for galaxy ACO85J004153.51-092943.8.

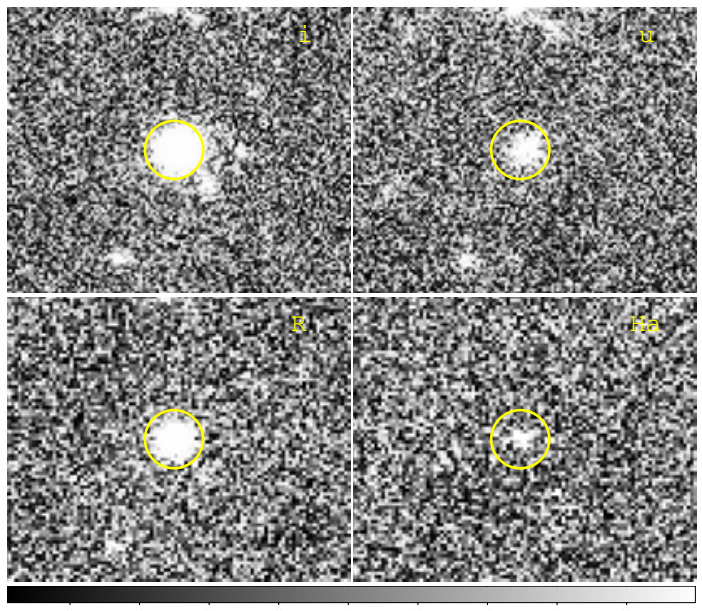

Fig. A.54. Same as Fig. 1 for galaxy ACO85J004154.04-094510.2. 
G. Boué et al.: Filament of Abell 85, Online Material p 16

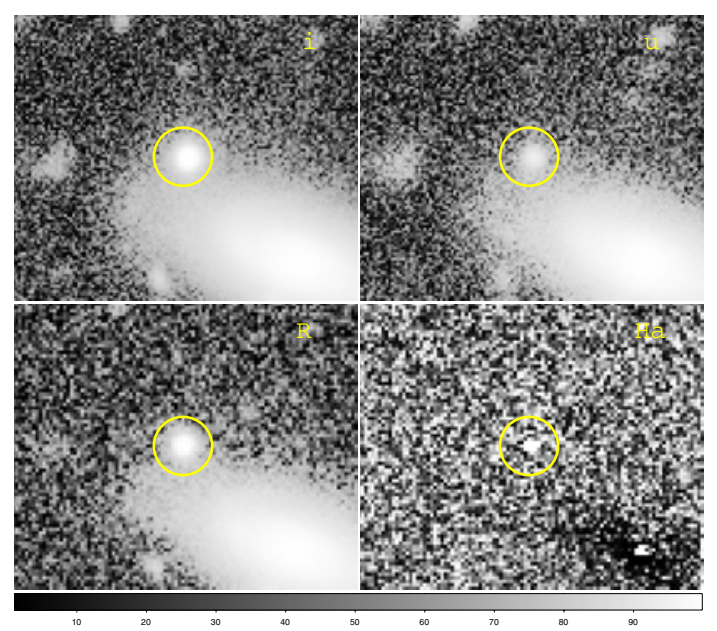

Fig. A.55. Same as Fig. 1 for galaxy ACO85J004157.86-093516.5.

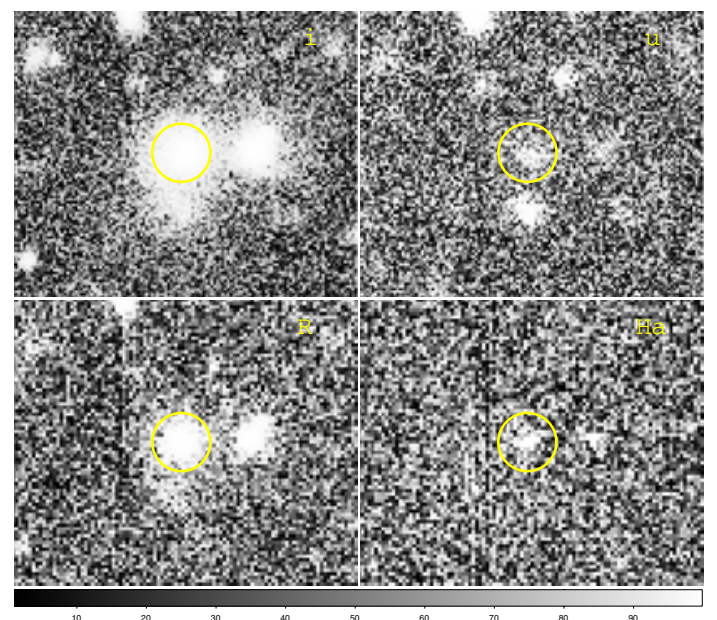

Fig. A.56. Same as Fig. 1 for galaxy ACO85J004158.81-092815.3.

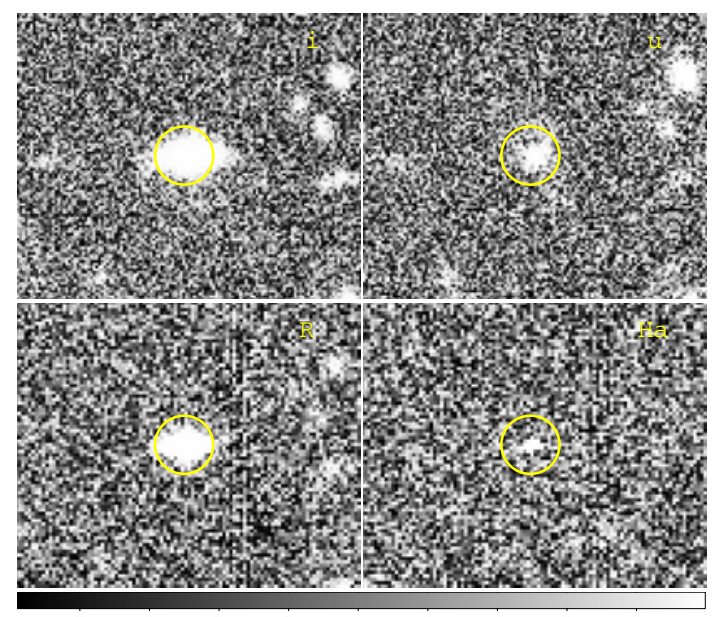

Fig. A.57. Same as Fig. 1 for galaxy ACO85J004159.36-093010.9.

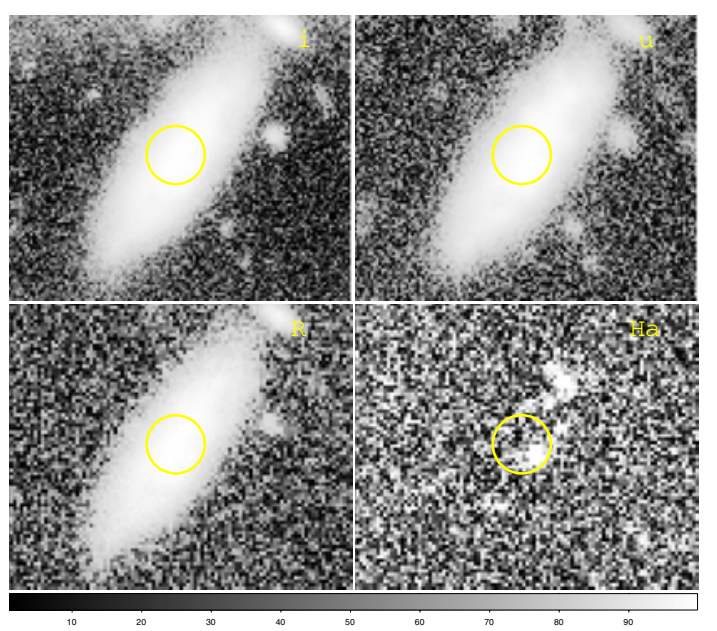

Fig. A.58. Same as Fig. 1 for galaxy ACO85J004159.84-094230.9.

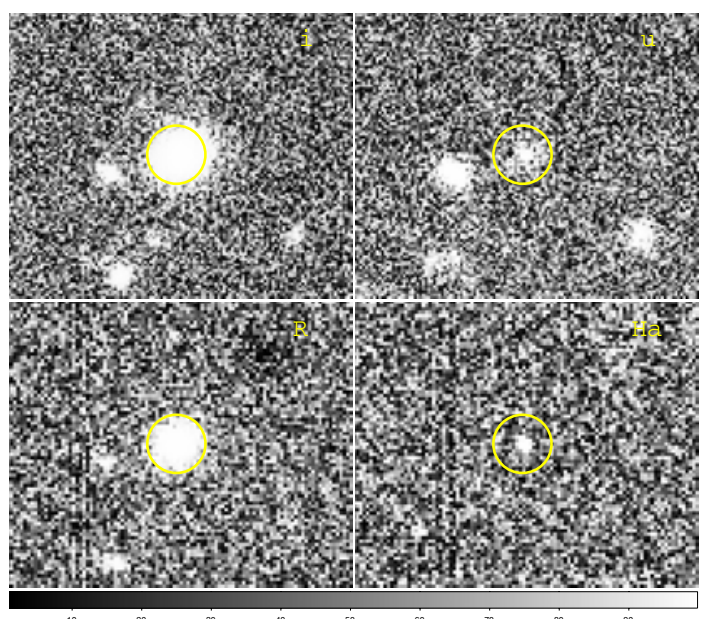

Fig. A.59. Same as Fig. 1 for galaxy ACO85J004202.99-093302.1.

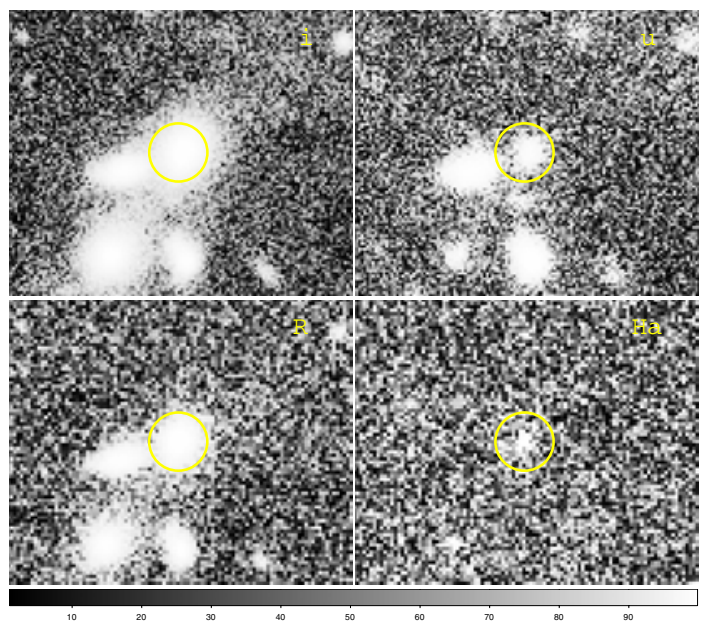

Fig. A.60. Same as Fig. 1 for galaxy ACO85J004204.90-094108.6. 
G. Boué et al.: Filament of Abell 85, Online Material p 17

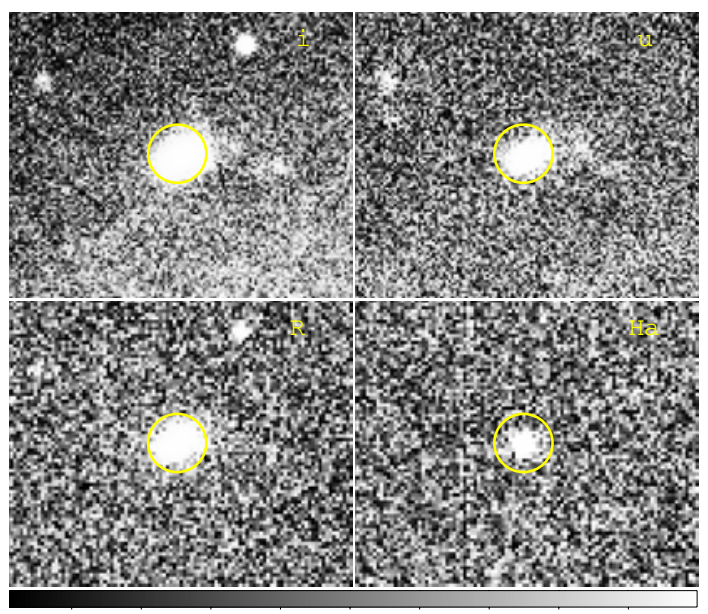

Fig. A.61. Same as Fig. 1 for galaxy ACO85J004205.15-093715.5.
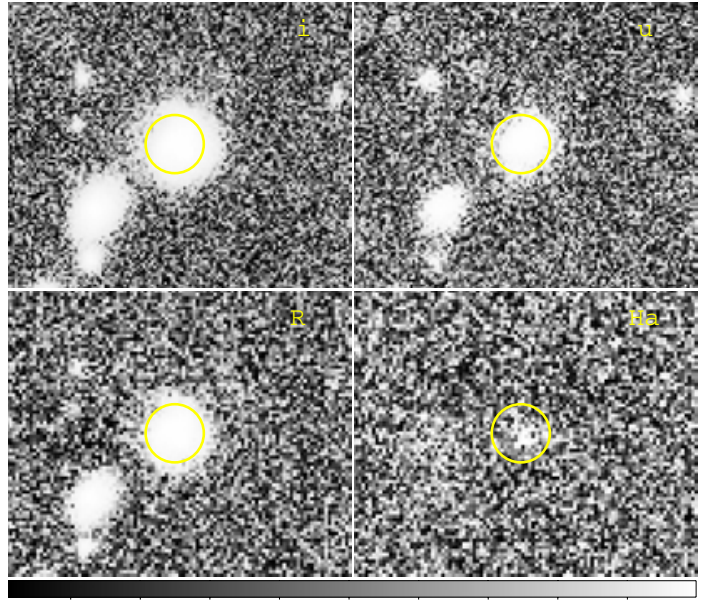

Fig. A.62. Same as Fig. 1 for galaxy ACO85J004205.67-094627.1.

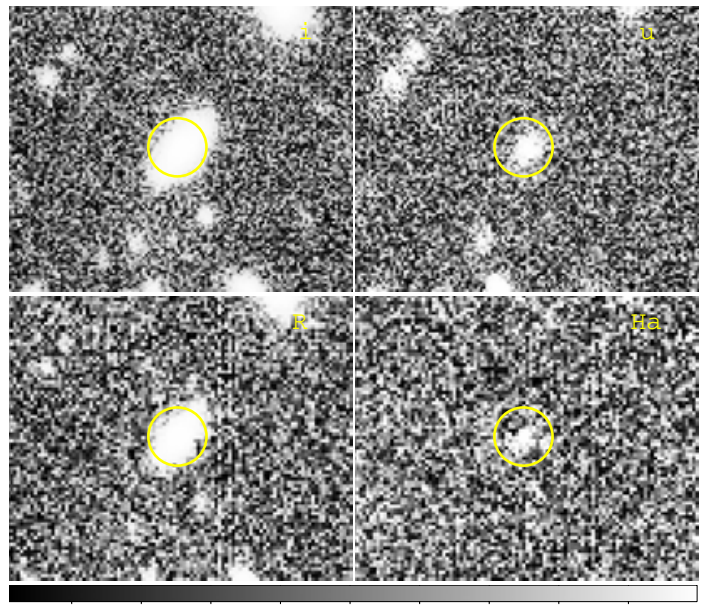

Fig. A.63. Same as Fig. 1 for galaxy ACO85J004205.79-093026.0.

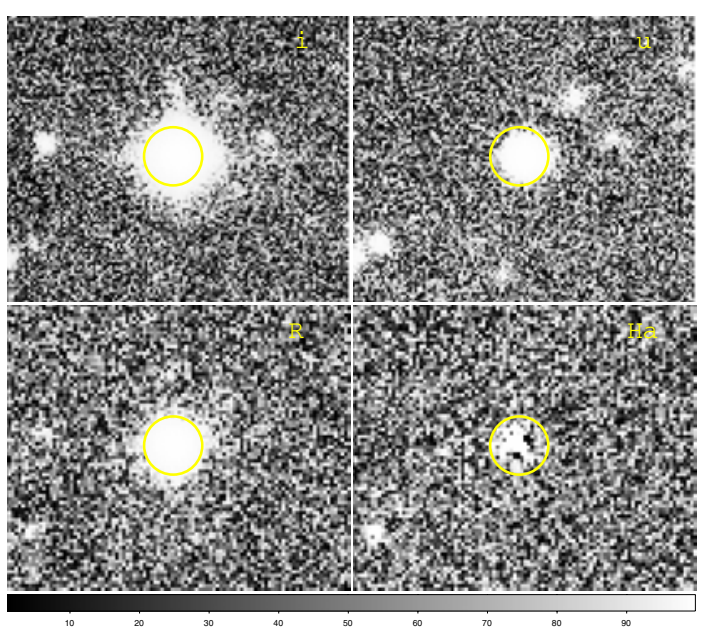

Fig. A.64. Same as Fig. 1 for galaxy ACO85J004205.86-094310.1.
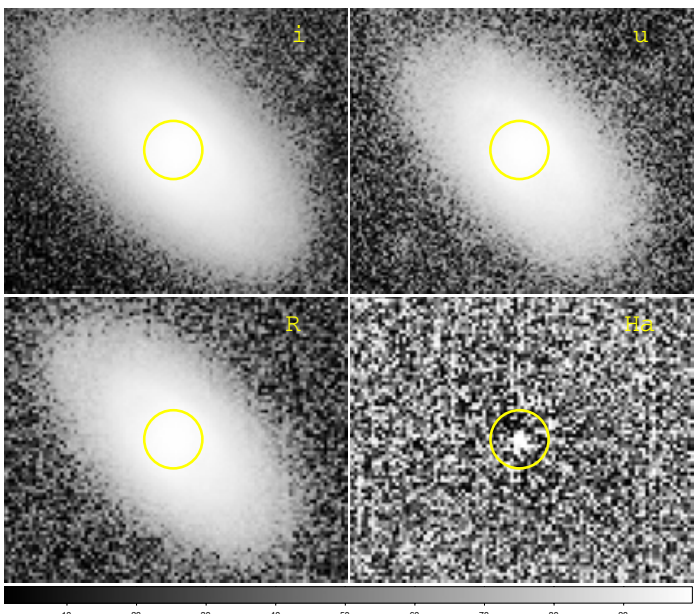

Fig. A.65. Same as Fig. 1 for galaxy ACO85J004206.02-093606.4.

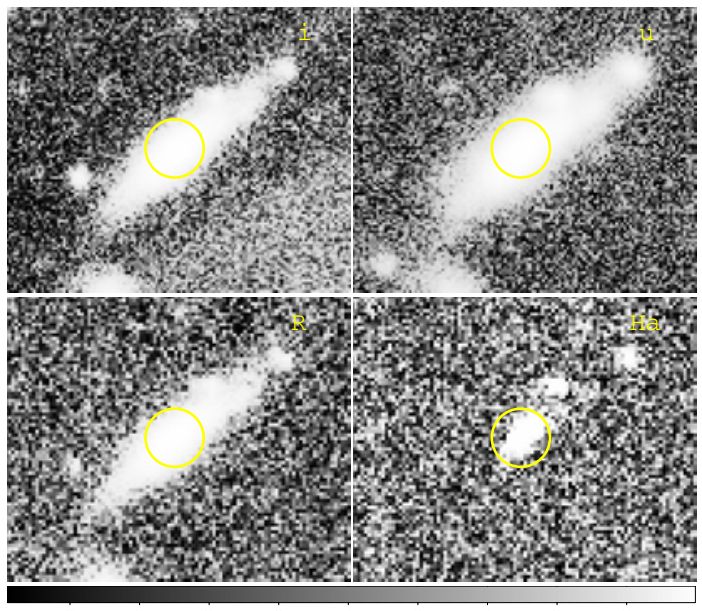

Fig. A.66. Same as Fig. 1 for galaxy ACO85J004207.26-093626.0. 
G. Boué et al.: Filament of Abell 85, Online Material p 18

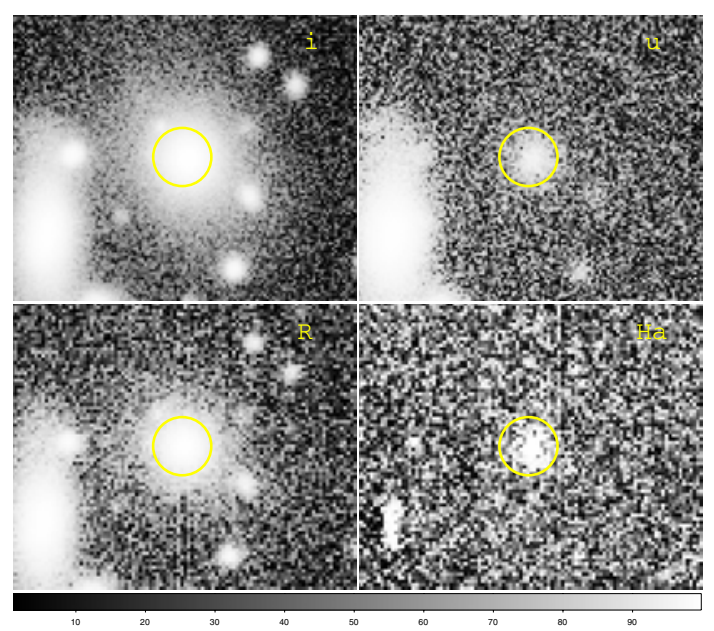

Fig. A.67. Same as Fig. 1 for galaxy ACO85J004207.71-093059.3.

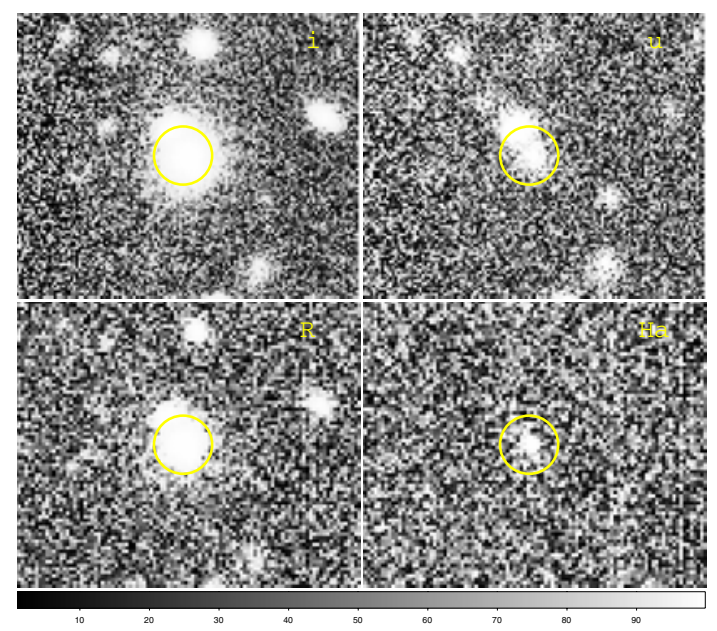

Fig. A.68. Same as Fig. 1 for galaxy ACO85J004208.27-092942.7.

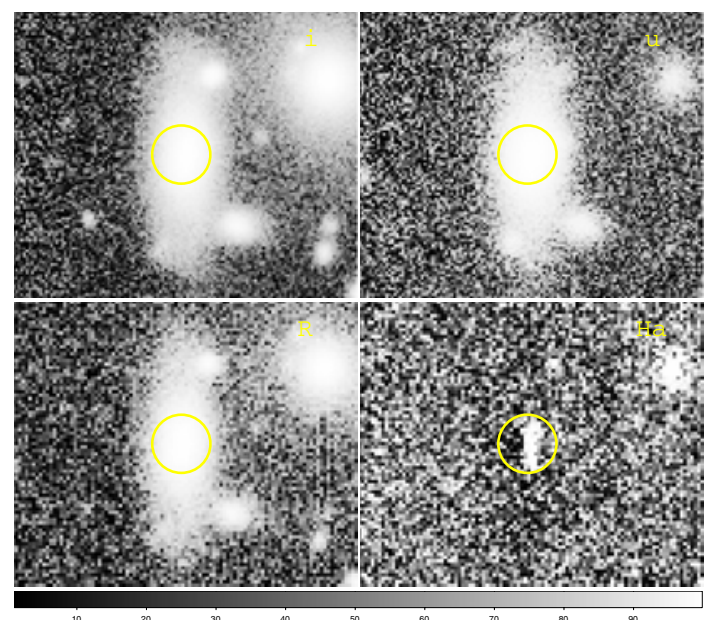

Fig. A.69. Same as Fig. 1 for galaxy ACO85J004208.36-093104.6.

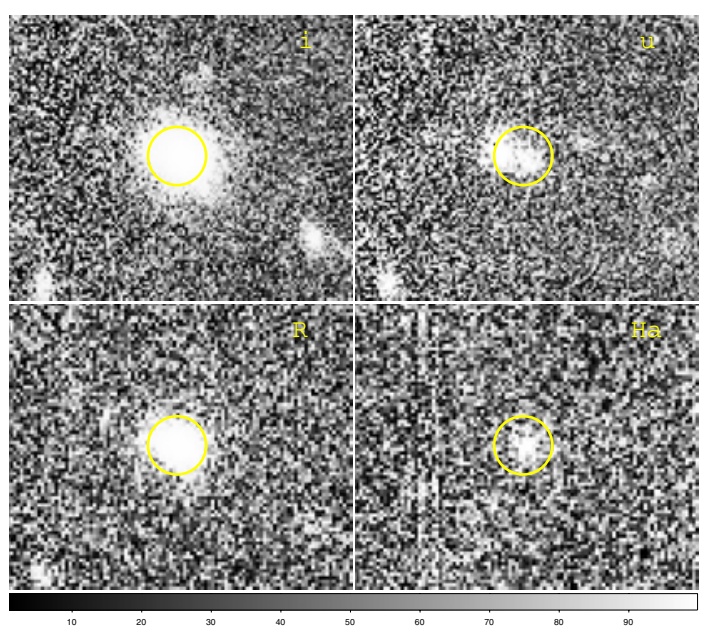

Fig. A.70. Same as Fig. 1 for galaxy ACO85J004208.67-093506.5.

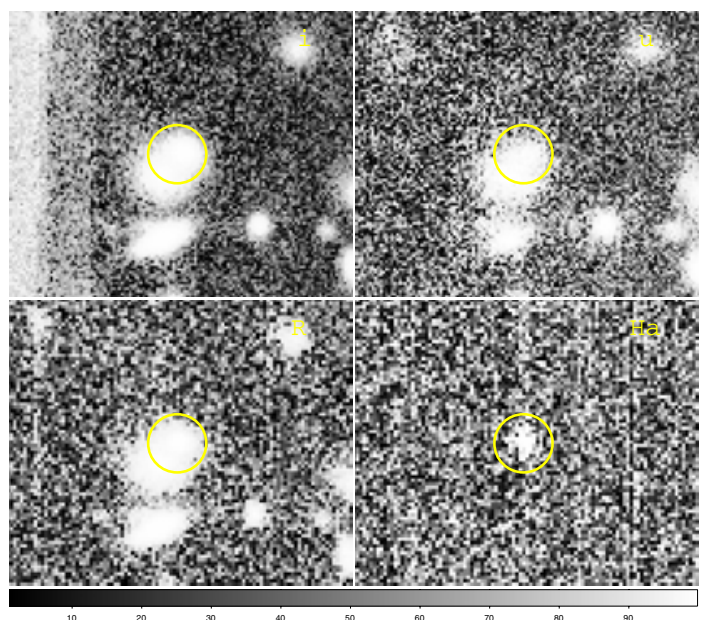

Fig. A.71. Same as Fig. 1 for galaxy ACO85J004209.19-094056.6.

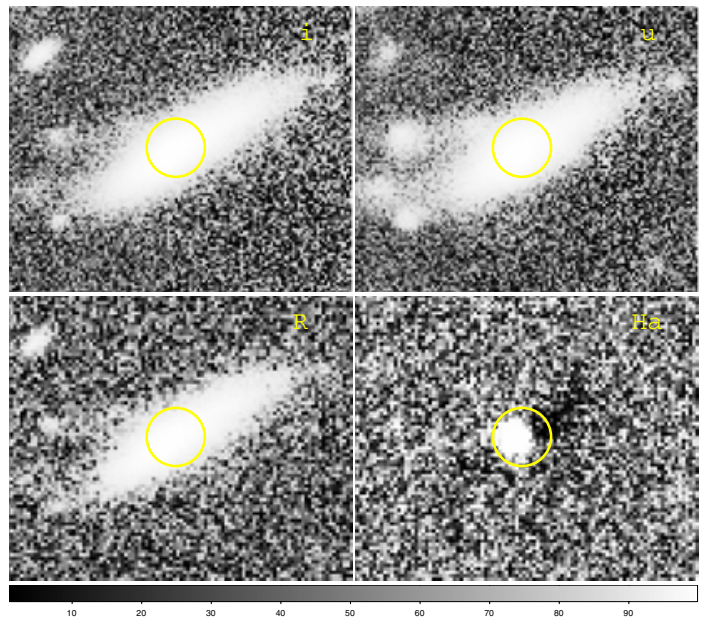

Fig. A.72. Same as Fig. 1 for galaxy ACO85J004209.81-092852.2. 
G. Boué et al.: Filament of Abell 85, Online Material p 19

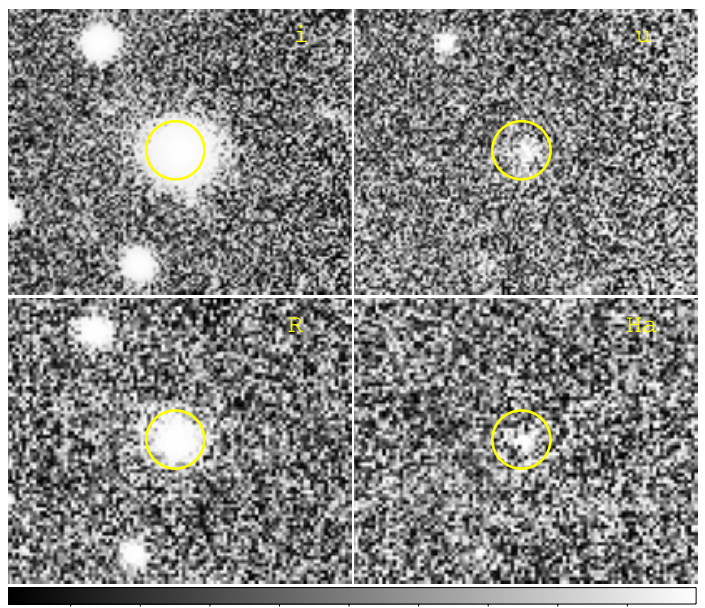

Fig. A.73. Same as Fig. 1 for galaxy ACO85J004210.63-093129.7.

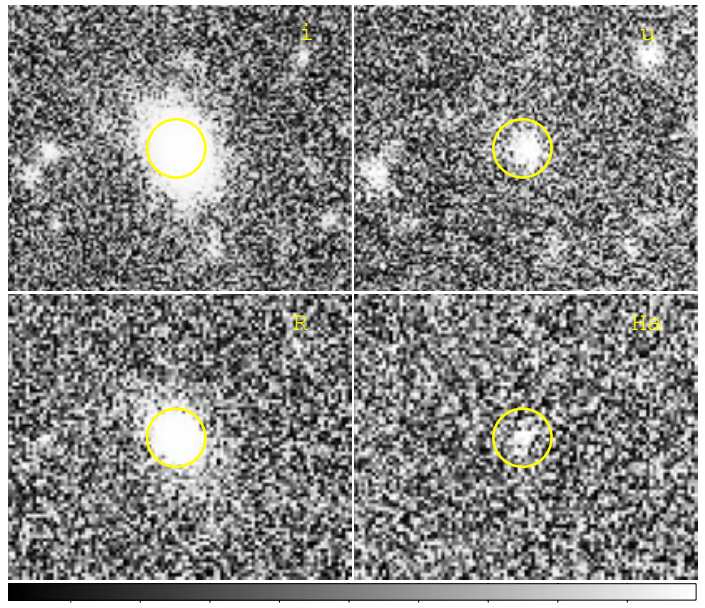

Fig. A.74. Same as Fig. 1 for galaxy ACO85J004214.92-092735.4.

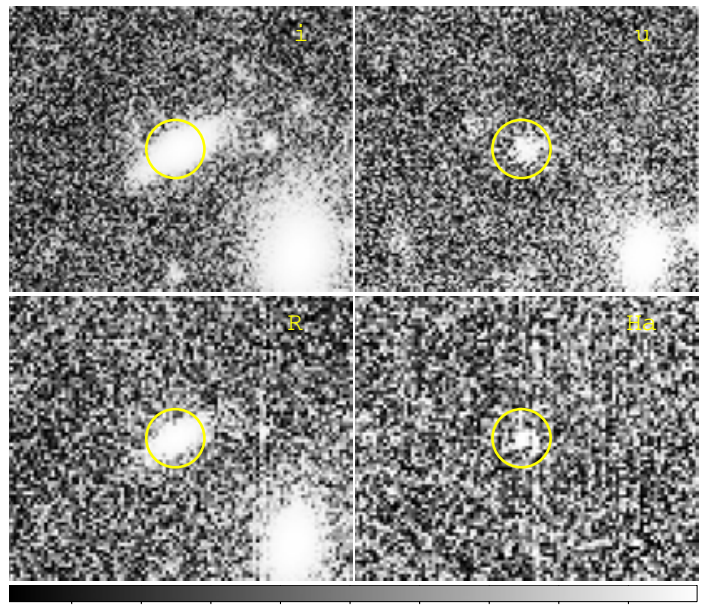

Fig. A.75. Same as Fig. 1 for galaxy ACO85J004215.64-094209.2.

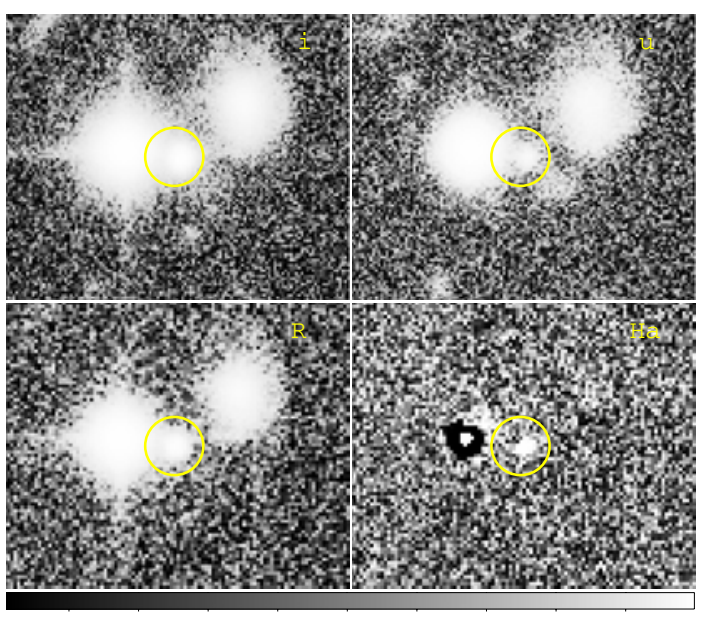

Fig. A.76. Same as Fig. 1 for galaxy ACO85J004216.93-093325.6.

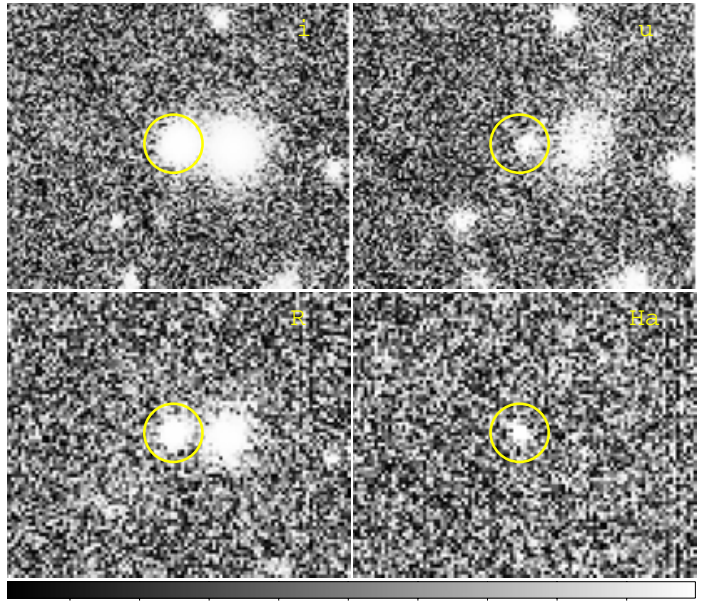

Fig. A.77. Same as Fig. 1 for galaxy ACO85J004217.94-093620.9.

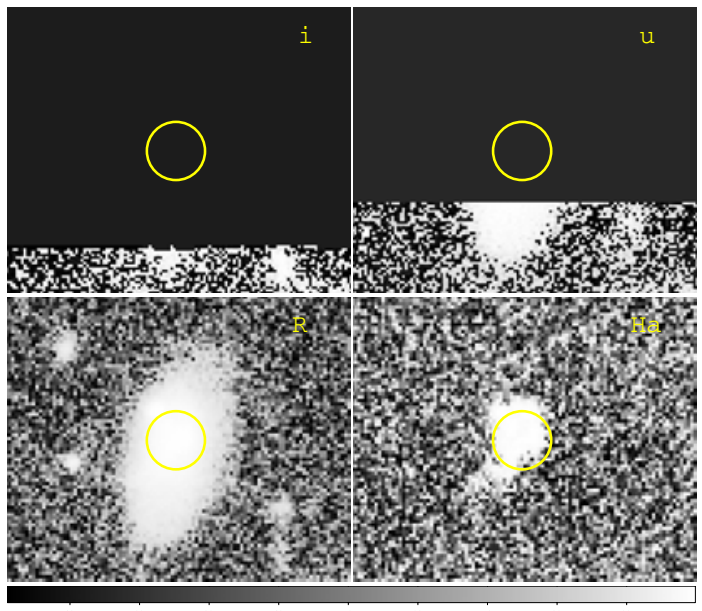

Fig. A.78. Same as Fig. 1 for galaxy ACO85J004218.47-093912.1. 
G. Boué et al.: Filament of Abell 85, Online Material p 20

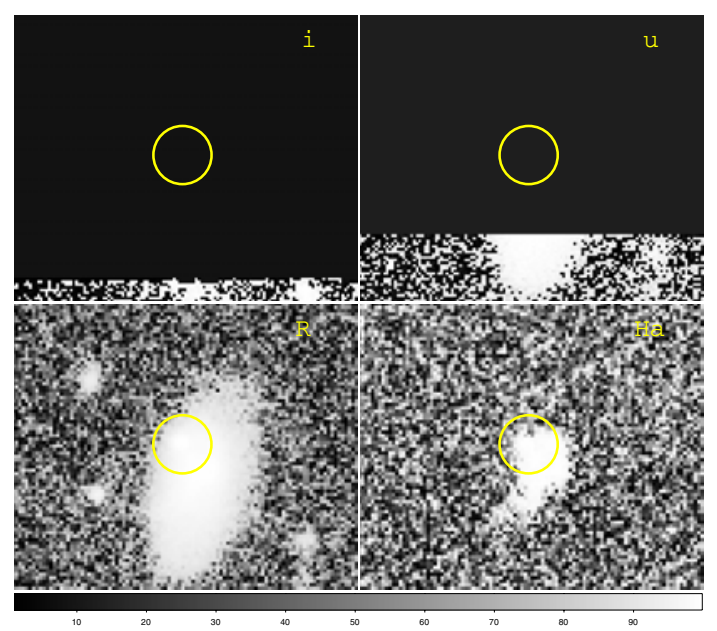

Fig. A.79. Same as Fig. 1 for galaxy ACO85J004218.55-093910.2.
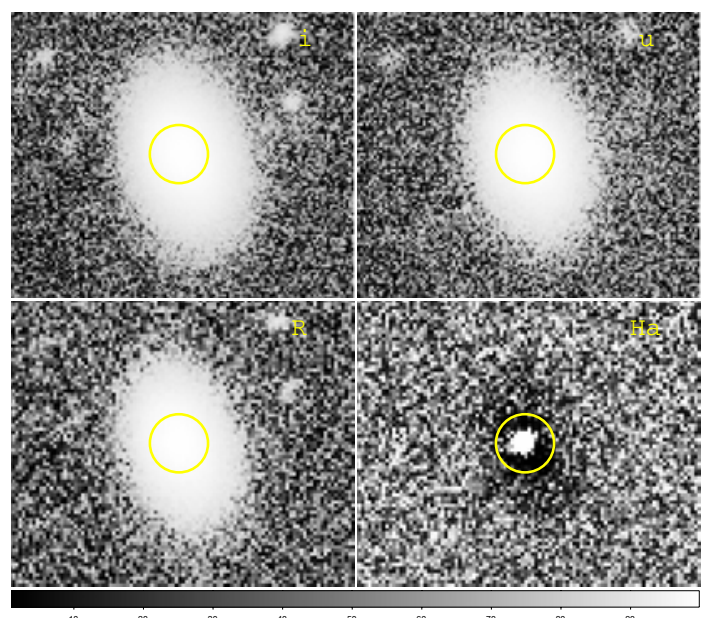

Fig. A.80. Same as Fig. 1 for galaxy ACO85J004219.89-092527.5.

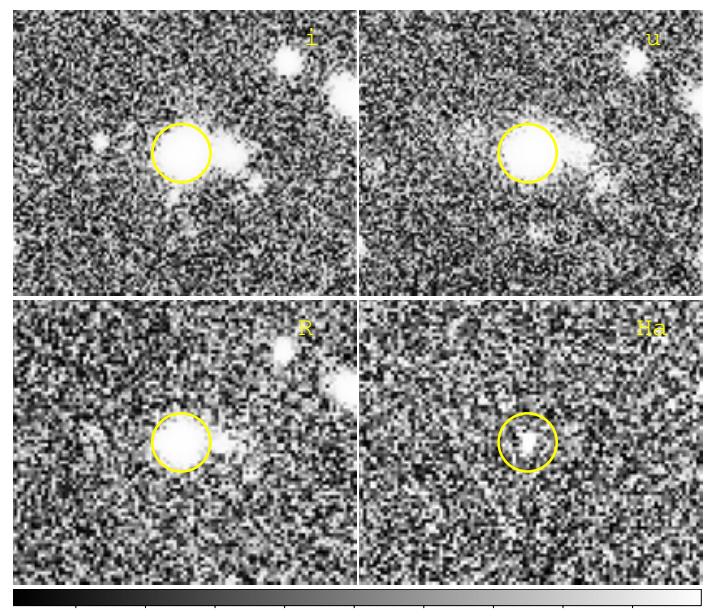

Fig. A.81. Same as Fig. 1 for galaxy ACO85J004220.58-093526.4.

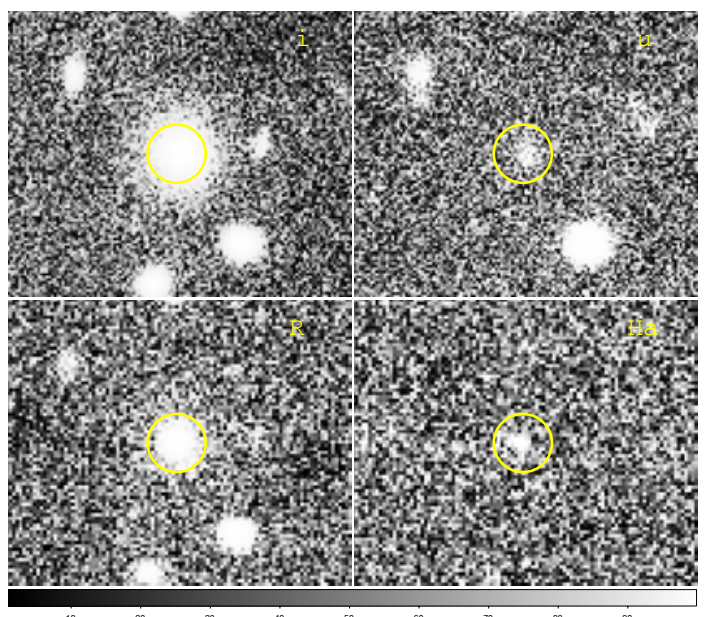

Fig. A.82. Same as Fig. 1 for galaxy ACO85J004220.87-094517.5.

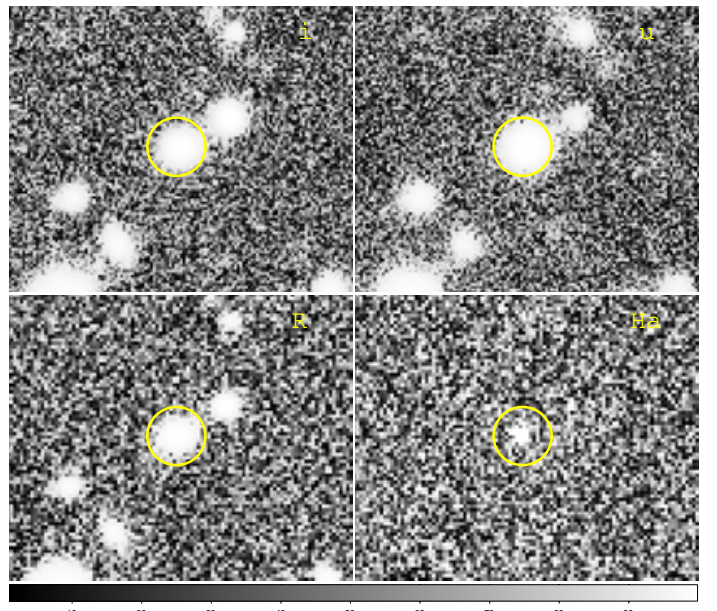

Fig. A.83. Same as Fig. 1 for galaxy ACO85J004224.68-092716.2.

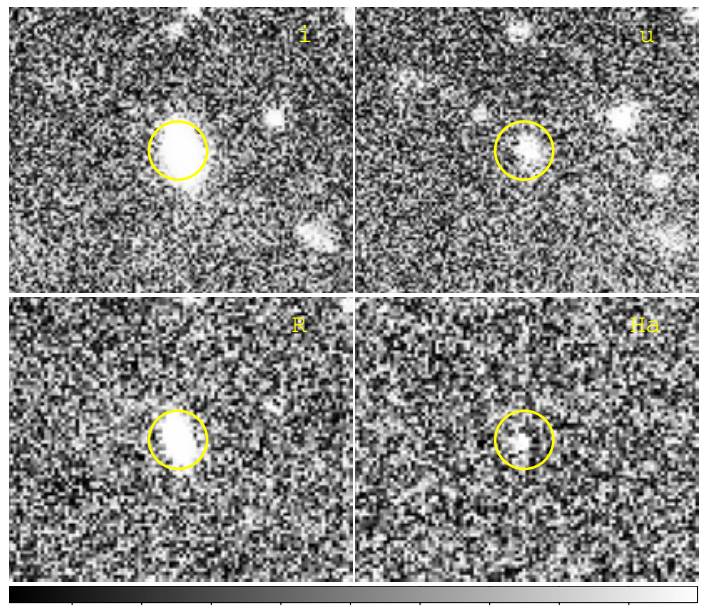

Fig. A.84. Same as Fig. 1 for galaxy ACO85J004224.74-093741.3. 
G. Boué et al.: Filament of Abell 85, Online Material p 21

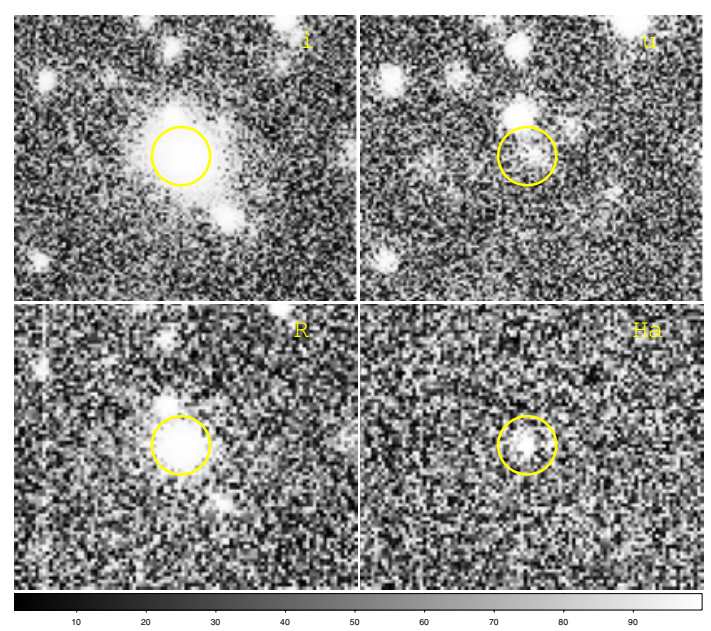

Fig. A.85. Same as Fig. 1 for galaxy ACO85J004225.48-093538.6.
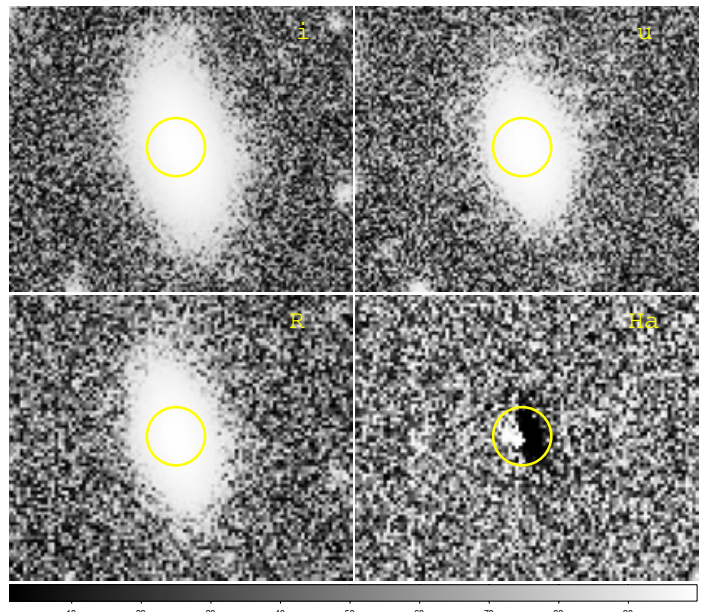

Fig. A.86. Same as Fig. 1 for galaxy ACO85J004225.54-093708.9.

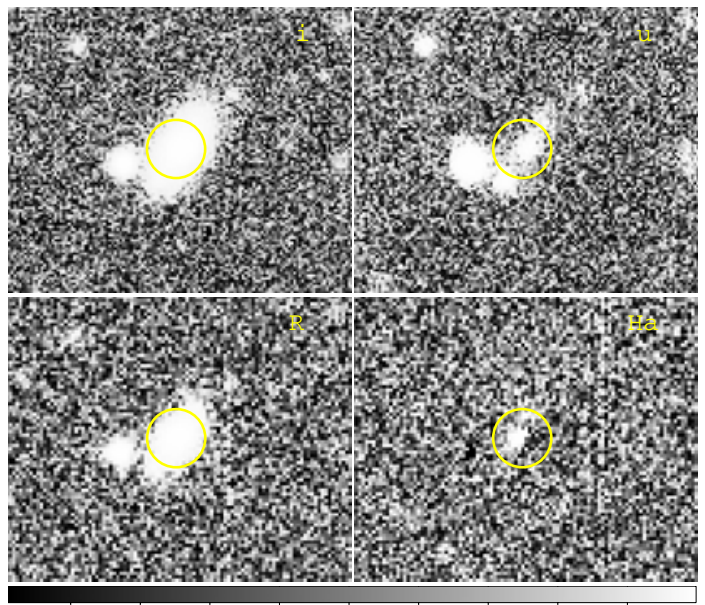

Fig. A.87. Same as Fig. 1 for galaxy ACO85J004226.35-093629.5.

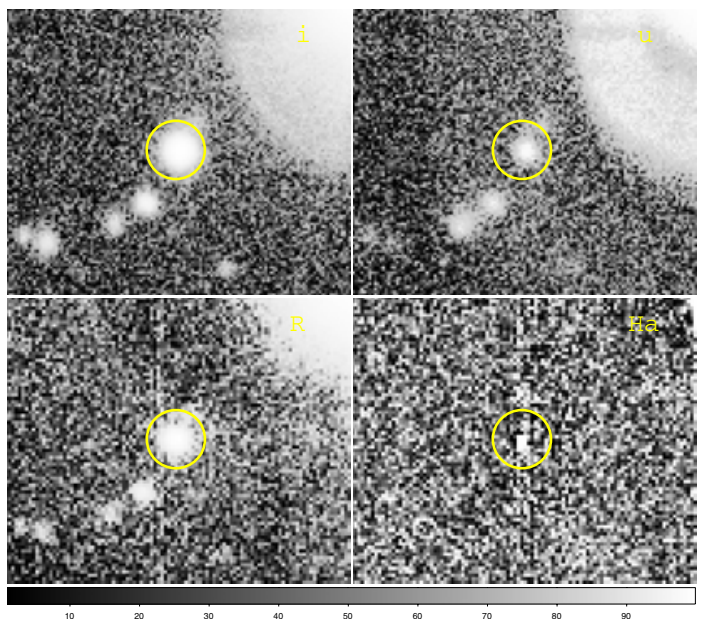

Fig. A.88. Same as Fig. 1 for galaxy ACO85J004227.26-093116.5.

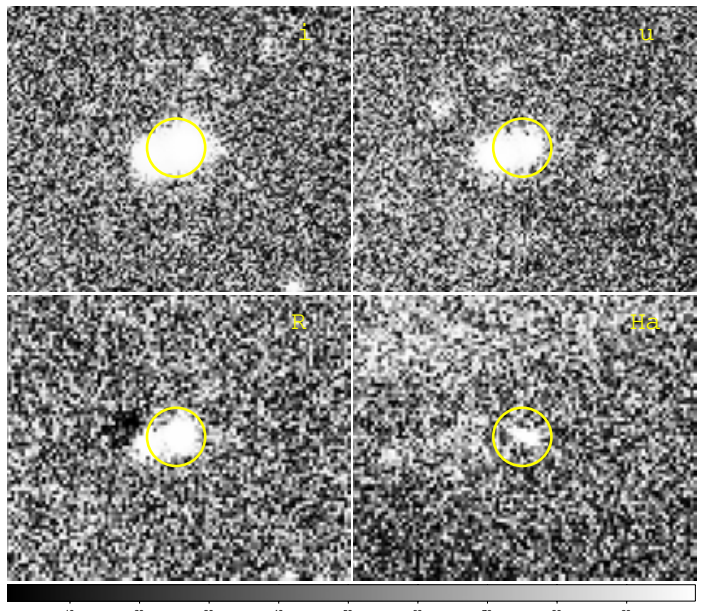

Fig. A.89. Same as Fig. 1 for galaxy ACO85J004227.58-095059.3.

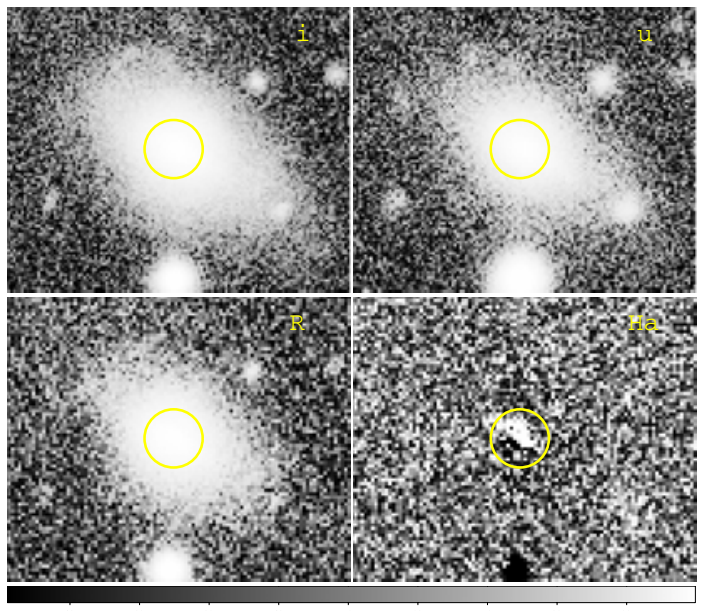

Fig. A.90. Same as Fig. 1 for galaxy ACO85J004228.38-094938.3. 
G. Boué et al.: Filament of Abell 85, Online Material p 22

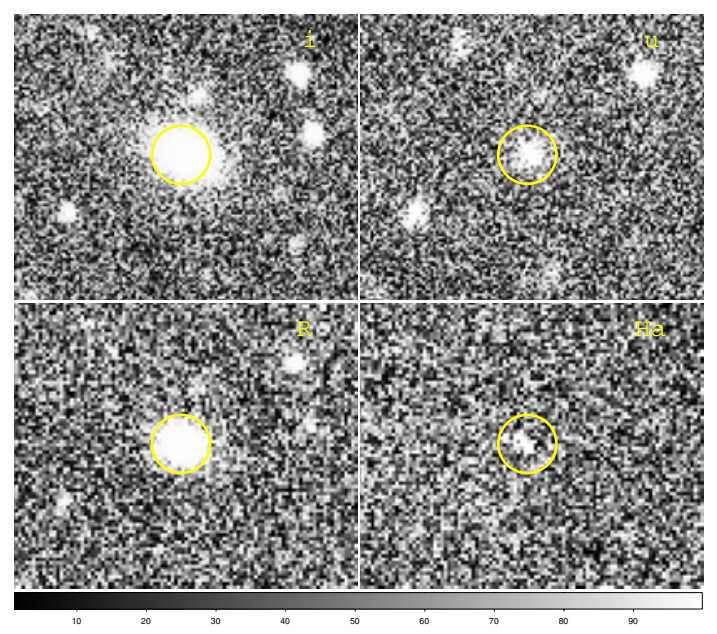

Fig. A.91. Same as Fig. 1 for galaxy ACO85J004228.83-094523.9.

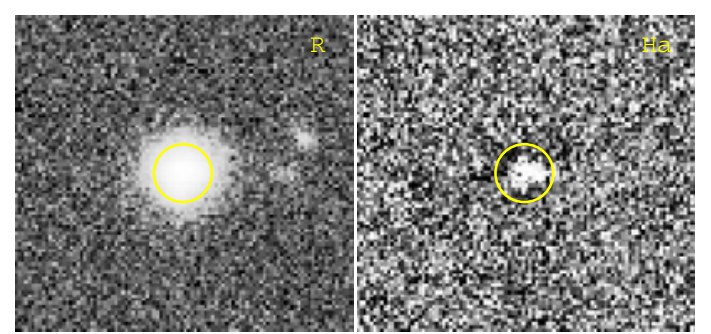

Fig. A.92. Same as Fig. 1 for galaxy ACO85J004232.84-092144.2.

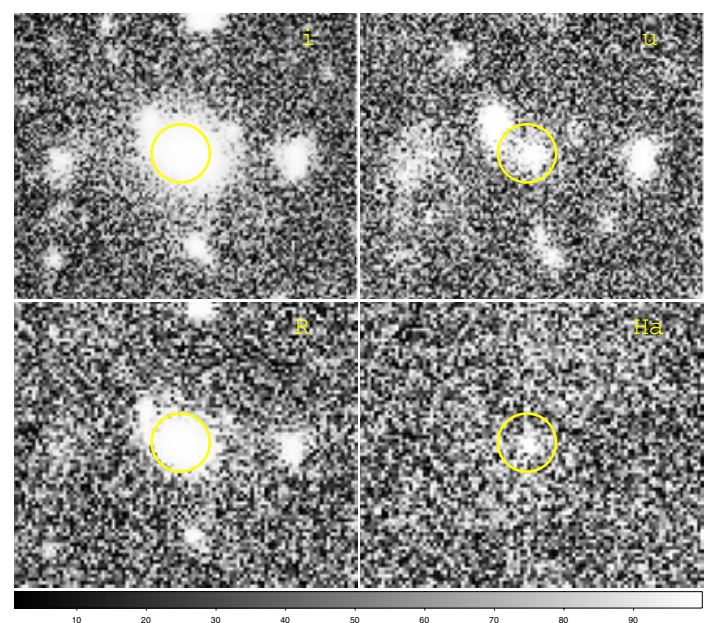

Fig. A.93. Same as Fig. 1 for galaxy ACO85J004233.31-094448.0.

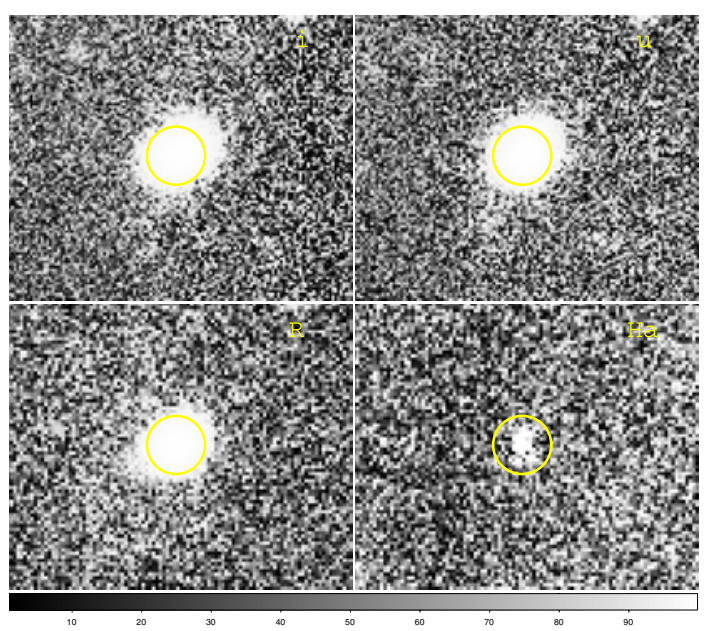

Fig. A.94. Same as Fig. 1 for galaxy ACO85J004236.76-094403.8.
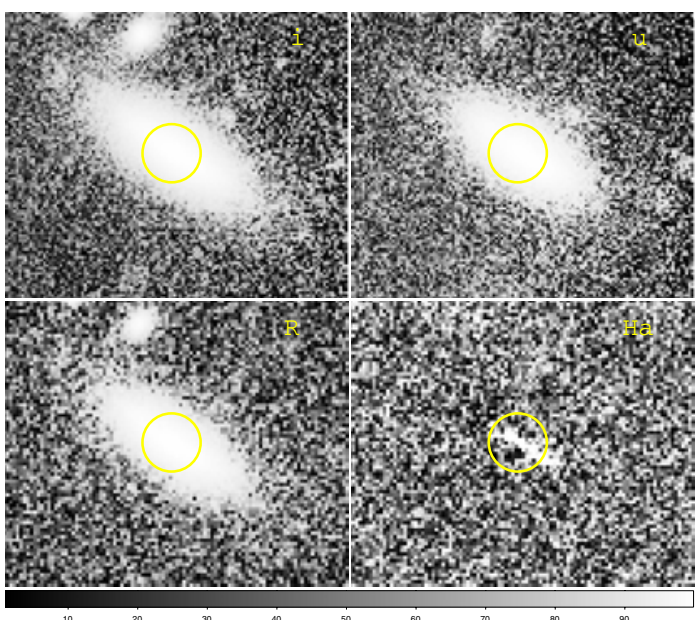

Fig. A.95. Same as Fig. 1 for galaxy ACO85J004237.07-094520.5.

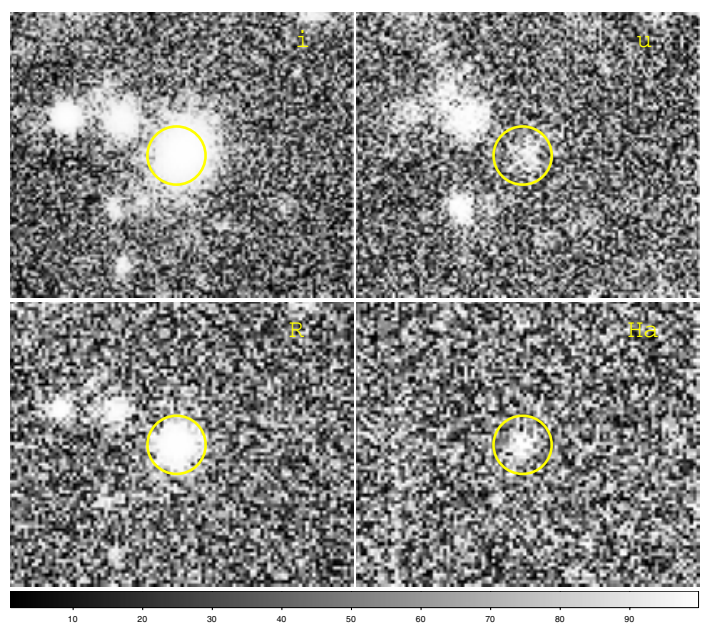

Fig. A.96. Same as Fig. 1 for galaxy ACO85J004238.03-093229.9. 
G. Boué et al.: Filament of Abell 85, Online Material p 23

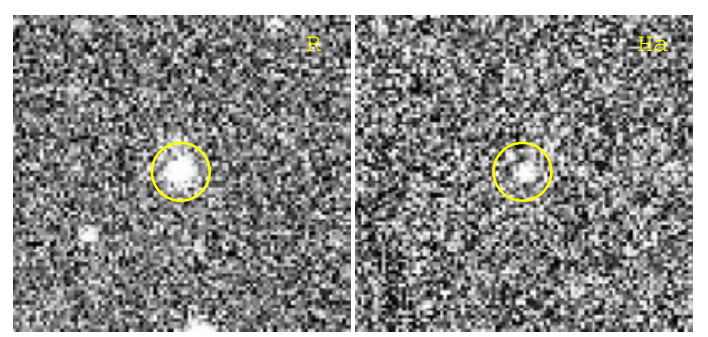

Fig. A.97. Same as Fig. 1 for galaxy ACO85J004242.24-092108.7.

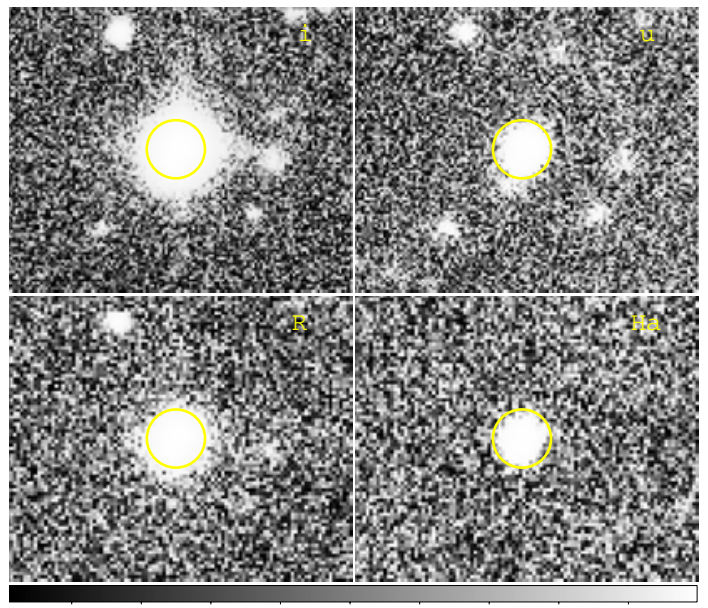

Fig. A.98. Same as Fig. 1 for galaxy ACO85J004242.54-094726.4.

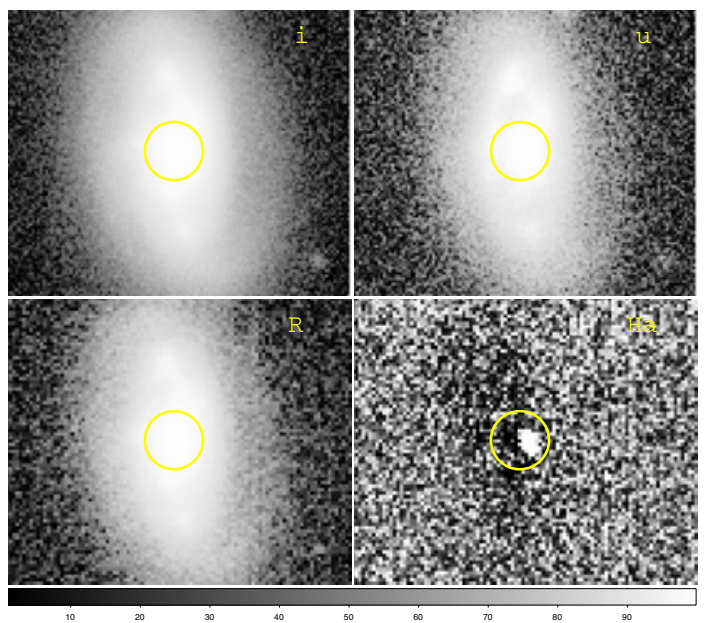

Fig. A.99. Same as Fig. 1 for galaxy ACO85J004243.90-094420.8.

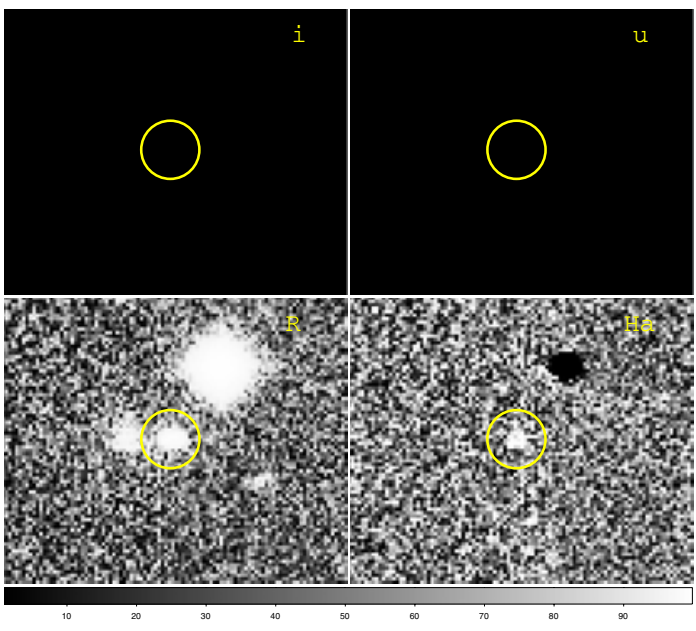

Fig. A.100. Same as Fig. 1 for galaxy ACO85J004245.68-092327.8.

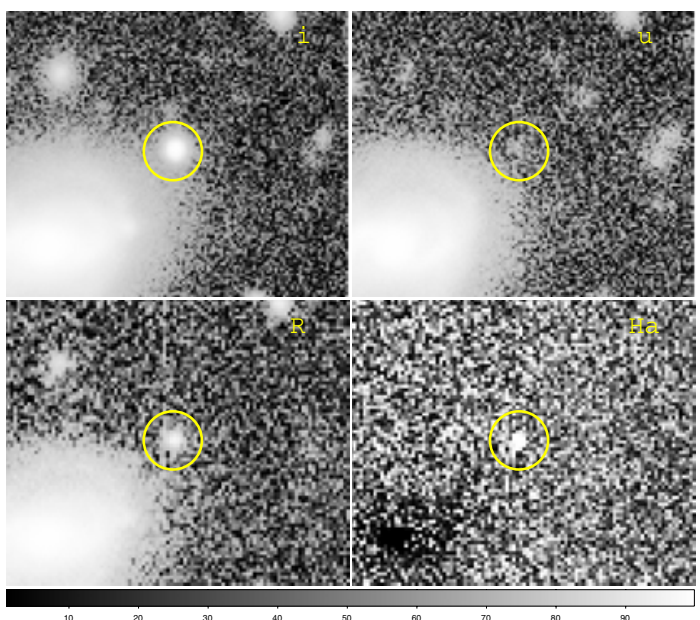

Fig. A.101. Same as Fig. 1 for galaxy ACO85J004247.85-092522.6. 
G. Boué et al.: Filament of Abell 85, Online Material p 24
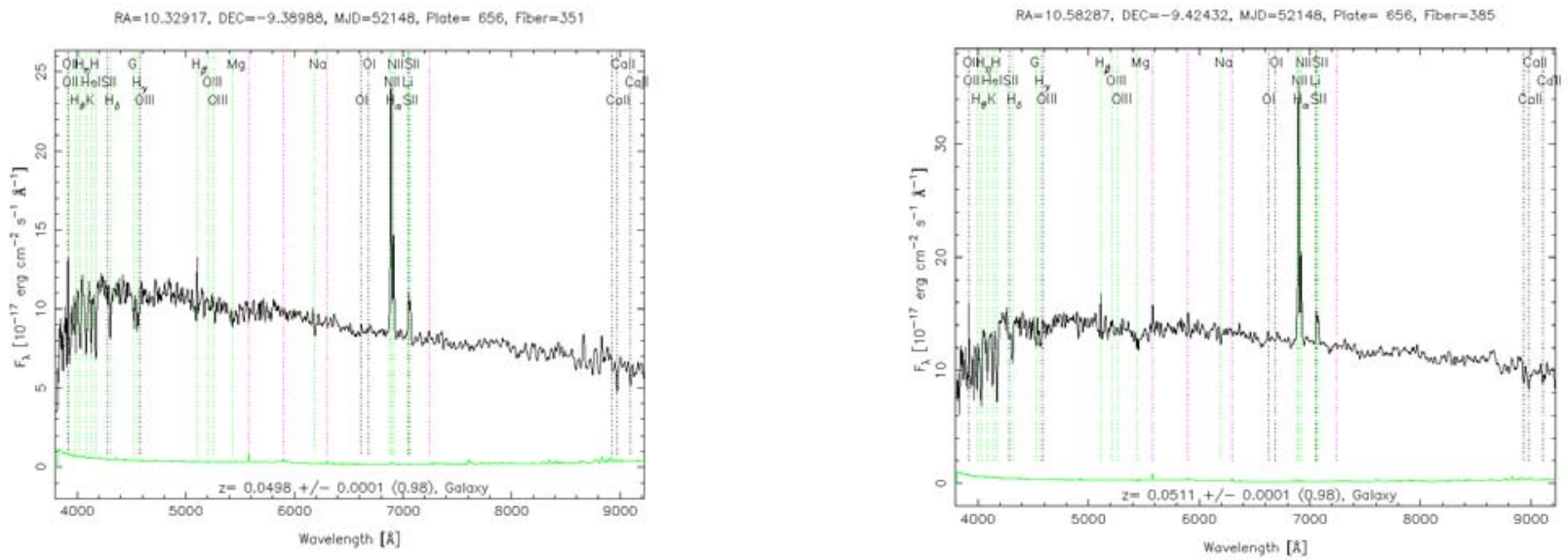

Fig. A.102. SDSS spectrum for galaxy ACO85J004119.01-092323.50.

Fig. A.105. SDSS spectrum for galaxy ACO85J004219.90-092527.55.
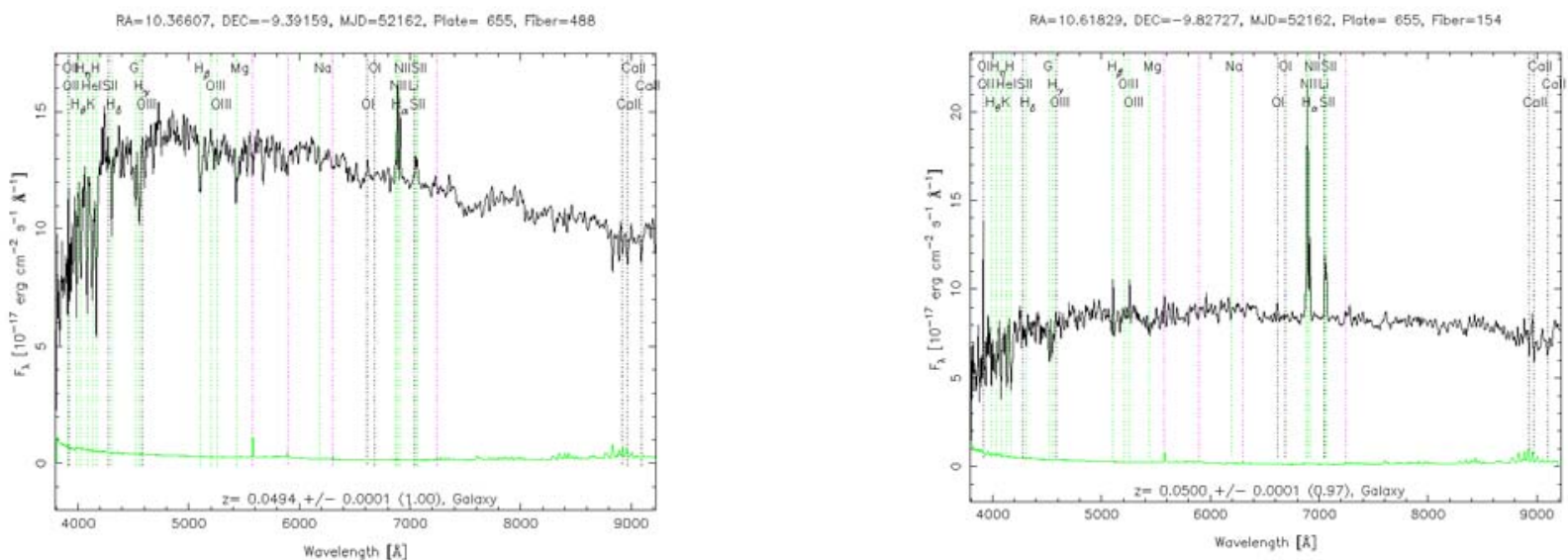

Fig. A.103. SDSS spectrum for galaxy ACO85J004127.86-092329.54.

Fig. A.106. SDSS spectrum for galaxy ACO85J004228.37-094938.28.
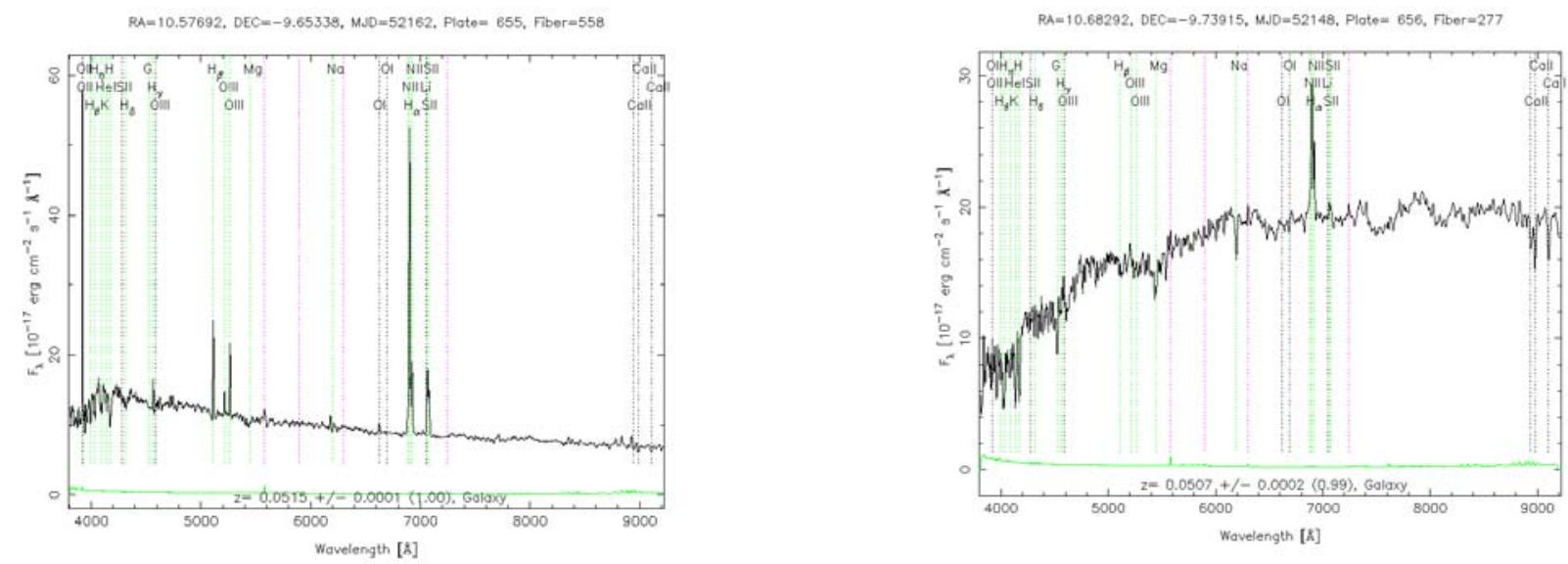

Fig. A.104. SDSS spectrum for galaxy ACO85J004218.46-093912.10.

Fig. A.107. SDSS spectrum for galaxy ACO85J004243.90-094420.83. 